\title{
Curvature-induced bunch self-interaction for an energy-chirped bunch in magnetic bends
}

\author{
Rui Li \\ Thomas Jefferson National Accelerator Facility, 12000 Jefferson Avenue, Newport News, Virginia 23606, USA
}

(Received 17 August 2007; published 14 February 2008)

\begin{abstract}
Within the realm of classical electrodynamics, the curvature-induced bunch collective interaction in magnetic bends can be studied using effective forces in the canonical formulation of the coherent synchrotron radiation (CSR) effect. As an application of this canonical formulation, in this paper, for an electron distribution moving ultrarelativistically in a bending system, the dynamics of the particles in the distribution is derived from the Hamiltonian of the particles in terms of the bunch internal coordinates. The consequent Vlasov equation manifests explicitly how the phase-space distribution is perturbed by the effective CSR forces. In particular, we study the impact of an initial linear energy chirp of the bunch on the behavior of the effective longitudinal CSR force, which arises due to the modification of the retardation relation as a result of the energy-chirping-induced longitudinal-horizontal correlation of the bunch distribution (bunch tilt) in dispersive regions. Our study clearly demonstrates the time delay (or retardation) of the behavior of the effective longitudinal CSR force on a bunch in responding to the change of the bunch length in a magnetic bend. Our result also shows that the effective longitudinal CSR force for a bunch under full compression can have sensitive dependence on the transverse position of the test particle in the bunch for certain parameter regimes.
\end{abstract}

DOI: 10.1103/PhysRevSTAB.11.024401

PACS numbers: 41.60.Ap, 29.27.Bd, 41.75.Ht

\section{INTRODUCTION}

In linear accelerators or linac drivers for free-electron lasers, often a linear energy chirp, or a linear correlation between a particle's energy and its longitudinal position in a bunch, is imposed on a bunch by accelerating the bunch off-crest in an rf cavity. Transporting such an energychirped bunch through a bending system allows the manipulation of the bunch length via the correlation between path length and energy in dispersive regions. For example, a high peak current of an electron beam is often achieved by compressing the high-charge, properly energy-chirped electron bunches using a magnetic bunch-compression chicane. When such an electron bunch with linear energy chirp $(\delta-z$ correlation) goes through a dispersive region ( $x-\delta$ correlation), such as that in a magnetic chicane, a horizontal-longitudinal $(x-z)$ correlation, or bunch tilt, is introduced to the bunch distribution.

The effect of curvature-induced bunch self-interaction, often called the coherent synchrotron radiation (CSR) effect, on microbunching instability in a bunchcompression chicane has been extensively studied both analytically [1-3] and numerically [4]. As an approximation, these studies are based on the longitudinal CSR wakefield [5-7] obtained for a rigid-line bunch, where the longitudinal charge distribution at time $t$ is obtained by projecting an actual tilted bunch at $t$ onto the designed circular orbit and the longitudinal CSR force is calculated assuming the longitudinal distribution thus obtained has remained unchanged for all the previous times $\left(t^{\prime}<t\right)$. The effective CSR forces were also analyzed earlier [8] for a nontilted thin bunch. Compared to the case of a nontilted or projected bunch, the $x-z$ correlation of the bunch distribution modifies the geometry of particle interaction with respect to the direction of particle motion, which consequently modifies the retardation solution and the CSR interaction force. The numerical results of the effect of the $x-z$ correlation on the CSR interaction were first presented by Dohlus [9] for a thin (negligible intrinsic transverse and longitudinal spread) Gaussian bunch. In this study we focus on the analysis of the effect of the $x-z$ correlation on the behavior of the CSR interaction forces for a two-dimensional Gaussian bunch with arbitrary rootmean square of the intrinsic phase-space spread.

Since the variation of bunch tilt depends closely on the beam phase-space transport along the beam line of interest, to study the bunch tilt effect on the curvature-induced collective forces, we choose a general approach by formulating the bunch collective interaction on a curved orbit as a self-consistent dynamical system for an arbitrary initial phase-space distribution. This is done by first developing the equations of motion in Sec. II based on the Hamiltonian of the particles in the bunch distribution, and then constructing the equation for the evolution of the phase-space distribution in Sec. III, where the role of the effective collective forces on the perturbation of the phase-space distribution is explicitly shown. We then focus on the study of the CSR interaction for a 2D energy-chirped bunch on a circular orbit with the unperturbed-source approximation. This includes the formulation of the retarded potentials (Sec. IV), the solutions of the retardation relation (Sec. V), and the analysis of the effective longitudinal CSR force (Sec. VI). The analytical results obtained in this paper are in good agreement with Dohlus' numerical results for a moderately tilted thin bunch. We also present results for a tilted thick bunch, as obtained from numerical integration, which manifests strong two-dimensional effects around full compression. 
It is well known that, when a charge distribution moves ultrarelativistically along a curved orbit, the particles experience the "centrifugal space-charge force" $F^{\mathrm{CSCF}}$ in the radial direction [10], as a result of the nonperfect cancellation between the electric and magnetic fields in the Lorentz force. Meanwhile, the particles with radial offset from the design orbit also experience the "noninertial space-charge force" $F^{\mathrm{NSCF}}$ in the longitudinal direction [11]. The singular contribution of nearby-particle interactions to these forces could cause complication for one to analyze the curvature-induced collective interactions for a tilted bunch. In this paper, this complication is eased by using the canonical formulation developed in Ref. [12], since the canonical formulation can exhibit explicitly the cancellation (or anticorrelation) of the effects of $F^{\mathrm{CSCF}}$ and of the potential change (which is partly contributed by $F^{\mathrm{NSCF}}$ ) on the bunch transverse dynamics. Consequently, the effective CSR forces, which are the net residual of this cancellation, are recognized as the source of perturbation to the phase-space distribution. Compared to $F^{\mathrm{CSCF}}$ and $F^{\mathrm{NSCF}}$, the energy-independent parts of the effective forces are usually dominated by the nonlocal contributions of particle interaction. As shown in this paper, both the effective CSR force and the centrifugal space-charge force may show strong dependence on a particle's transverse position. However, the former is caused by the retardation under a special geometry of interaction, while the latter is due to the singular contribution of nearby-particle interaction.

Even though in this paper our focus is limited to the energy-independent part of the longitudinal effective force for a tilted bunch under CSR interaction within a single magnetic bend, the basic equations developed in Secs. II and III provide a framework for further self-consistent analysis or simulation of the CSR effect on the dynamics of an energy-chirped bunch in a general magnetic bending system. In particular, for a tilted bunch, the approach used in Secs. IV, V, and VI can be straightforwardly extended to the study of the single-bend transverse effective CSR force, as well as the effective forces in the transient regime involving straight sections on the entrance to or exit from a circular orbit. In light of the fact that the full CSR and space-charge effect in a bending system can only be studied self-consistently by computer simulations, we hope that the study in this paper can provide some analytical understanding of the interaction process, and can serve as a benchmark to simulations for certain simplified cases.

Note that this study assumes the continuity of the bunch phase-space distribution. Therefore topics related to discrete-particle effects which are present in actual physical processes, such as the single-particle radiation reaction, or the discrete-particle collisional effects for radiative particles, are beyond the scope of this paper.

\section{FORMULATION OF THE CSR PROBLEM AS A DYNAMICAL SYSTEM}

Our goal in this section is to formulate particle dynamics for an electron distribution moving ultrarelativistically on a curved design orbit under collective interaction in free space (impedance effects due to boundary condition are not included). With a brief discussion of the dynamics in the Cartesian coordinates in the laboratory frame, we review the equations of motion based on the canonical formulation [12] in the Frenet-Serret coordinates along the design orbit, using path length $s$ as the independent variable. Then by writing both the equations of motion and the effective forces in terms of our chosen beam phasespace parameters, we formulate the problem as a dynamical system [see Eq. (29)]. This provides the foundation for setting up the equation for the evolution of the phase-space distribution (see Sec. III), and allows us to analyze the curvature-induced effective forces for an energy-chirped bunch in magnetic bends (Secs. IV, V, and VI).

\section{A. Dynamics in a Cartesian coordinate system}

Consider an electron bunch moving relativistically in an external electromagnetic field and undergoing collective interaction. For a fixed Cartesian coordinate system, the position vector is $\mathbf{r}=X \mathbf{i}+Y \mathbf{j}+Z \mathbf{k}$. The dynamics of a charged particle (with respect to $t$ ) in the bunch can be studied using the Hamiltonian

$$
H=\sqrt{\left(c P_{x}-e A_{x}^{\text {tot }}\right)^{2}+\left(c P_{y}-e A_{y}^{\text {tot }}\right)^{2}+\left(c P_{z}-e A_{z}^{\text {tot }}\right)^{2}+m^{2} c^{4}}+e \Phi^{\text {tot }}
$$

Here $\mathbf{P}$ is the canonical momentum conjugate to $\mathbf{r}$, and

$$
\Phi^{\mathrm{tot}}=\Phi^{\mathrm{ext}}+\Phi^{\mathrm{col}}, \quad \mathbf{A}^{\mathrm{tot}}=\mathbf{A}^{\mathrm{ext}}+\mathbf{A}^{\mathrm{col}}
$$

are the scalar and vector potentials on the charged particle, with $\left(\Phi^{\mathrm{ext}}, \mathbf{A}^{\mathrm{ext}}\right)$ the potentials related to the external design fields, and $\left(\Phi^{\mathrm{col}}, \mathbf{A}^{\mathrm{col}}\right)$ the potentials due to collective electromagnetic interactions among particles in the charge distribution. Let $\mathbf{r}_{0}(t ; \zeta)$ denote the trajectory of a source particle, where $\zeta=[\mathbf{r}(0), \dot{\mathbf{r}}(0)]$ is the source particle's initial phase-space coordinates at $s=0$ which is used here to label the orbit of the individual particle. Let us further denote $f_{0}(\zeta)$ as the distribution of the electron bunch over the phase space $\zeta$ at $s=0$. From the retarded potential generated by a single source particle [13],

$$
A^{\mu}(x)=2 e \int d \tau V^{\mu}(\tau) \theta\left[x_{0}-r_{0}(\tau)\right] \delta\left\{[x-r(\tau)]^{2}\right\}
$$

for $V^{\mu}(\tau)=(\gamma c, \gamma \dot{\mathbf{r}})$, one can obtain the collective potentials (in the Lorentz gauge) on a test particle generated by all the source particles in the electron distribution: 


$$
\left\{\begin{array}{l}
\Phi^{\mathrm{col}}(\mathbf{r}, t)=2 e c \int d \zeta f_{0}(\zeta) \int d t_{r} \theta\left(t-t_{r}\right) \delta\left[c^{2}\left(t-t_{r}\right)^{2}-\left|\mathbf{r}-\mathbf{r}_{\mathbf{0}}\left(t_{r}, \zeta\right)\right|^{2}\right] \\
\mathbf{A}^{\mathrm{col}}(\mathbf{r}, t)=2 e \int d \zeta f_{0}(\zeta) \int d t_{r} \dot{\mathbf{r}}\left(t_{r}\right) \theta\left(t-t_{r}\right) \delta\left[c^{2}\left(t-t_{r}\right)^{2}-\left|\mathbf{r}-\mathbf{r}_{\mathbf{0}}\left(t_{r}, \zeta\right)\right|^{2}\right]
\end{array}\right.
$$

where $\theta(x)$ is the Heaviside step function, and $\delta(x)$ is the Dirac-delta function.

\section{B. Dynamics in the bunch internal coordinate system}

We now let the path length $s$ along the design orbit be the independent variable, and summarize the equations of motion in the Frenet-Serret coordinates [12]. Here the position vector of a particle is

$$
\mathbf{r}(x, y, s)=x \mathbf{e}_{x}(s)+y \mathbf{e}_{y}(s)+\mathbf{r}_{0}(s),
$$

with $\mathbf{r}_{0}(s)$ denoting the vector for the reference orbit. Let $H$ be the canonical energy conjugate to $t$, and $\mathcal{P}_{x}$ and $\mathcal{P}_{y}$ be the transverse canonical momentums conjugate to $x$ and $y$. The Hamiltonian for an electron in the bunch conjugate to $s$ is then

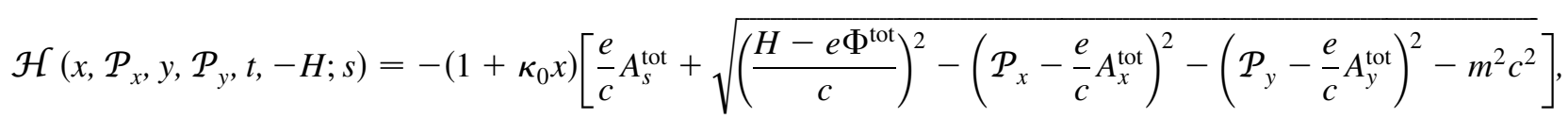

where $\kappa_{0}(s)=1 / R_{0}(s)$ is the curvature of the design orbit at $s$, and the scalar and vector potentials are the sum of the external and collective potentials

$$
\Phi^{\mathrm{tot}}=\Phi^{\mathrm{ext}}+\Phi^{(\mathrm{F})}, \quad \mathbf{A}^{\mathrm{tot}}=\mathbf{A}^{\mathrm{ext}}+\mathbf{A}^{(\mathrm{F})} .
$$

Here $\Phi^{(\mathrm{F})}$ and $\mathbf{A}^{(\mathrm{F})}$ are the collective interaction potentials in Eq. (4) converted to the Frenet-Serret coordinates using Eq. (5):

$$
\begin{aligned}
& \Phi^{(\mathrm{F})}(x, y, s, t)=\Phi^{\mathrm{col}}[\mathbf{r}(x, y, s), t], \\
& \mathbf{A}^{(\mathrm{F})}(x, y, s, t)=\mathbf{A}^{\mathrm{col}}[\mathbf{r}(x, y, s), t],
\end{aligned}
$$

and similar changes of variables are done for $\Phi^{\mathrm{ext}}$ and $\mathbf{A}^{\mathrm{ext}}$. The Frenet components of $\mathbf{A}^{(\mathrm{F})}(x, y, z, s)$ are then

$$
\begin{aligned}
& A_{x}^{(\mathrm{F})}(x, y, t, s)=\mathbf{A}^{(\mathrm{F})} \cdot \mathbf{e}_{x}(s), \\
& A_{y}^{(\mathrm{F})}(x, y, t, s)=\mathbf{A}^{(\mathrm{F})} \cdot \mathbf{e}_{y}(s), \\
& A_{s}^{(\mathrm{F})}(x, y, z, s)=\mathbf{A}^{(\mathrm{F})} \cdot \mathbf{e}_{s}(s) .
\end{aligned}
$$

Normalizing the Hamiltonian in Eq. (6) by $\gamma_{0} m c=E_{0} / c$, with $E_{0}$ being the design beam energy, one gets

$$
\tilde{\mathcal{H}}\left(x, \tilde{\mathcal{P}}_{x}, y, \tilde{\mathcal{P}}_{y}, c t,-\tilde{H} ; s\right)=-\left(1+\kappa_{0} x\right)\left[\tilde{A}_{s}^{\mathrm{tot}}+\sqrt{\left(\tilde{H}-\tilde{\Phi}^{\mathrm{tot}}\right)^{2}-\left(\tilde{\mathcal{P}}_{x}-\tilde{A}_{x}^{\mathrm{tot}}\right)^{2}-\left(\tilde{\mathcal{P}}_{y}-\tilde{A}_{y}^{\mathrm{tot}}\right)^{2}-\gamma_{0}^{-2}}\right]
$$

with

$$
\left\{\tilde{\mathcal{H}}, \tilde{H}, \tilde{\mathcal{P}}_{x}, \tilde{\mathcal{P}}_{y}\right\}=\frac{1}{\gamma_{0} m c}\left\{\mathcal{H}, \frac{H}{c}, \mathcal{P}_{x}, \mathcal{P}_{y}\right\}
$$

and

$$
\left\{\tilde{\mathbf{A}}^{\mathrm{tot}}, \tilde{\Phi}^{\mathrm{tot}}\right\}=\frac{e}{\gamma_{0} m c^{2}}\left\{\mathbf{A}^{\mathrm{tot}}, \Phi^{\mathrm{tot}}\right\}
$$

In the rest of this paper, we will assume $A_{x}^{\mathrm{ext}}=A_{y}^{\mathrm{ext}}=$ $\Phi^{\mathrm{ext}}=0$. A canonical transformation from $(c t,-\tilde{H})$ to $\left(z, \delta_{H}\right)$ can be performed using the generating function [14]

$$
F_{3}(-\tilde{H}, z, s)=(s-z) \sqrt{\tilde{H}^{2}-\gamma_{0}^{-2}}+z,
$$

which gives

$$
\begin{aligned}
\delta_{H} & =-\frac{\partial F_{3}}{\partial z}=\sqrt{\tilde{H}^{2}-\gamma_{0}^{-2}}-1, \\
c t & =\frac{\partial F_{3}}{\partial \tilde{H}}=\frac{s-z}{\beta_{H}}, \\
\tilde{\mathcal{K}} & =\tilde{\mathcal{H}}+\frac{\partial F_{3}}{\partial s},
\end{aligned}
$$

for $\beta_{H}=\sqrt{1-\left(\gamma_{0} \tilde{H}\right)^{-1}}$. Let us define the normalized potentials, with variables changed according to Eq. (14), as

$$
\begin{aligned}
& \left\{\tilde{\Phi}, \tilde{A}_{x, y, s}, \tilde{\mathcal{A}}_{s}^{\mathrm{ext}}\right\}\left(x, y, z, \delta_{H}, s\right) \\
& \quad=\left\{\tilde{\Phi}^{(\mathrm{F})}, \tilde{A}_{x, y, s}^{(\mathrm{F})},\left(1+\kappa_{0} x\right) \tilde{A}_{s}^{\mathrm{ext}}\right\}\left(x, y, s, t=\frac{s-z}{\beta_{H}\left(\delta_{H}\right) c}\right),
\end{aligned}
$$

where $\tilde{\mathcal{A}}_{s}^{\text {ext }}=\left(1+\kappa_{0} x\right) A_{s}^{\text {ext }}$ is the canonical external longitudinal vector potential, and 


$$
\begin{aligned}
\beta_{H}\left(\delta_{H}\right) & =\left[1-\frac{1}{\gamma_{0}^{2}\left(1+\delta_{H}\right)^{2}+1}\right]^{1 / 2} \\
& \simeq \beta_{0}+\frac{\delta_{H}}{\gamma_{0}^{2}}+\frac{1}{2 \gamma_{0}^{4}} .
\end{aligned}
$$

Applying Eq. (10) to the new Hamiltonian $\tilde{\mathcal{K}}$ in Eq. (14), and expanding the square root in Eq. (10) to the second order of the small quantities

$$
\delta_{H},\left(\tilde{\mathcal{P}}_{x}-\tilde{A}_{x}\right),\left(\tilde{\mathcal{P}}_{y}-\tilde{A}_{y}\right), 1 / \gamma_{0}^{2},
$$

we get

$$
\tilde{\mathcal{K}}\left(x, \tilde{\mathcal{P}}_{x}, y, \tilde{\mathcal{P}}_{y}, z, \delta_{H} ; s\right) \simeq-\tilde{\mathcal{A}}_{s}^{\mathrm{ext}}-\kappa_{0} x\left(1+\delta_{H}\right)-\left(1+\kappa_{0} x\right)\left[\left(\tilde{A}_{s}-\tilde{\Phi}\right)-\frac{\left(\tilde{\mathcal{P}}_{x}-\tilde{A}_{x}\right)^{2}+\left(\tilde{\mathcal{P}}_{y}-\tilde{A}_{y}\right)^{2}}{2}-\frac{\tilde{\Phi}}{2 \gamma_{0}^{2}}\right] .
$$

The Hamilton's equation for $\left(x, \tilde{\mathcal{P}}_{x}, y, \tilde{\mathcal{P}}_{y}, z, \delta_{H}\right)$ can then be obtained after applying the potentials in Eq. (15) to $\tilde{\mathcal{K}}$ in Eq. (18):

$$
\begin{gathered}
\frac{d x}{d s}=\left(1+\kappa_{0} x\right)\left(\tilde{\mathcal{P}}_{x}-\tilde{A}_{x}\right), \\
\frac{d \mathcal{P}_{x}}{d s}=\left[\frac{\partial \tilde{\mathcal{A}}_{s}^{\mathrm{ext}}}{\partial x}+\kappa_{0}\left(1+\delta_{H}\right)\right]+\kappa_{0}\left[\left(\tilde{A}_{s}-\beta_{0} \tilde{\Phi}\right)-\frac{\left(\tilde{\mathcal{P}}_{x}-\tilde{A}_{x}\right)^{2}+\left(\tilde{\mathcal{P}}_{y}-\tilde{A}_{y}\right)^{2}}{2}-\frac{\tilde{\Phi}}{\gamma_{0}^{2}}\right] \\
+\left(1+\kappa_{0} x\right)\left[\frac{\partial\left(\tilde{A}_{s}-\beta_{0} \tilde{\Phi}\right)}{\partial x}+\left(\tilde{\mathcal{P}}_{x}-\tilde{A}_{x}\right) \frac{\partial \tilde{A}_{x}}{\partial x}+\left(\tilde{\mathcal{P}}_{y}-\tilde{A}_{y}\right) \frac{\partial \tilde{A}_{y}}{\partial x}-\frac{1}{\gamma_{0}^{2}} \frac{\partial \tilde{\Phi}}{\partial x}\right] \\
\frac{d y}{d s}=\left(1+\kappa_{0} x\right)\left(\tilde{\mathcal{P}}_{y}-\tilde{A}_{y}\right), \\
\frac{d \mathcal{P}_{y}}{d s}=\frac{\partial \tilde{\mathcal{A}}_{s}^{\mathrm{ext}}}{\partial y}+\left(1+\kappa_{0} x\right)\left[\frac{\partial\left(\tilde{A}_{s}-\beta_{0} \tilde{\Phi}\right)}{\partial y}+\left(\tilde{\mathcal{P}}_{x}-\tilde{A}_{x}\right) \frac{\partial \tilde{A}_{x}}{\partial y}+\left(\tilde{\mathcal{P}}_{y}-\tilde{A}_{y}\right) \frac{\partial \tilde{A}_{y}}{\partial y}-\frac{1}{\gamma_{0}^{2}} \frac{\partial \tilde{\Phi}}{\partial y}\right], \\
\frac{d H}{d s}=\frac{\partial \tilde{\mathcal{A}}_{s}^{\mathrm{ext}}}{\partial z}+\left(1+\kappa_{0} x\right)\left[\frac{\partial\left(\tilde{\mathcal{A}}_{s}-\beta_{0} \tilde{\Phi}\right)}{\partial z}+\left(\tilde{\mathcal{P}}_{x}-\tilde{A}_{x}\right) \frac{\partial \tilde{A}_{x}}{\partial z}+\left(\tilde{\mathcal{P}}_{y}-\tilde{A}_{y}\right) \frac{\partial \tilde{A}_{y}}{\partial z}-\frac{1}{\gamma_{0}^{2}} \frac{\partial \tilde{\Phi}}{\partial z}\right],
\end{gathered}
$$

with

$$
F_{z}=-\left(1+\kappa_{0} x\right)\left[\frac{\partial\left(\tilde{A}_{s}-\beta_{0} \tilde{\Phi}\right)}{\partial z}+\left(\tilde{\mathcal{P}}_{x}-\tilde{A}_{x}\right) \frac{\partial \tilde{A}_{x}}{\partial z}+\left(\tilde{\mathcal{P}}_{y}-\tilde{A}_{y}\right) \frac{\partial \tilde{A}_{y}}{\partial z}-\frac{1}{\gamma_{0}^{2}} \frac{\partial \tilde{\Phi}}{\partial z}\right] \frac{s-z}{\gamma_{0}^{2}}
$$

In the rest of this paper, we use the ultrarelativistic approximation $\beta_{H}\left(\delta_{H}\right)=\beta_{0}$ for Eq. (15). We will also assume

$$
\frac{d z}{d s}=-\kappa_{0} x+F_{z} \simeq-\kappa_{0} x
$$

This approximation is valid when the collective-forceinduced longitudinal slippage caused by the nonvanishing $\gamma_{0}^{-2}$ is negligible. A more specific condition is given in Eq. (B20).

Let us now use the external canonical vector potential [15]

$$
\begin{aligned}
\tilde{\mathcal{A}}_{s}^{\text {ext }}(x, y, s)= & -\left[\kappa_{0}(s) x+\left[\kappa_{0}^{2}(s)-\kappa_{1}(s)\right] \frac{x^{2}}{2}\right. \\
& \left.+\kappa_{1}(s) \frac{y^{2}}{2}\right]+\cdots
\end{aligned}
$$

to represent the external dipole and quadrupole magnetic fields

$$
\mathbf{B}^{\mathrm{ext}}=\frac{E_{0}}{e}\left\{\kappa_{1}(s) y \mathbf{e}_{x}(s)-\left[\kappa_{0}(s)+\kappa_{1}(s) x\right] \mathbf{e}_{y}(s)\right\}+\cdots .
$$

We can then deduce from Eqs. (19)-(24) the equations of motion for the noncanonical dynamical variables $\left(x, x^{\prime}, y, y^{\prime}, z, \delta_{H}\right)$. Since we are interested in the perturbation of the effective CSR force on the linear optics, by 
keeping only the linear terms for the design optics, we get

$$
\frac{d X}{d s}=Y(X, s) \equiv M(s) X+F^{[f]}(X, s)
$$

for

$$
\begin{aligned}
& X=\left(\begin{array}{c}
x \\
x^{\prime} \\
y \\
y^{\prime} \\
z \\
\delta_{H}
\end{array}\right), \\
& M(s)=\left(\begin{array}{cccccc}
0 & 1 & 0 & 0 & 0 & 0 \\
-k_{x}^{2}(s) & 0 & 0 & 0 & 0 & \kappa_{0}(s) \\
0 & 0 & 0 & 1 & 0 & 0 \\
0 & 0 & -k_{y}^{2}(s) & 0 & 0 & 0 \\
-\kappa_{0}(s) & 0 & 0 & 0 & 0 & 0 \\
0 & 0 & 0 & 0 & 0 & 0
\end{array}\right), \\
& F^{[f]}(X, s)=\left(\begin{array}{c}
0 \\
\tilde{F}_{x} \\
0 \\
\tilde{F}_{y} \\
0 \\
\tilde{F}_{H}
\end{array}\right) .
\end{aligned}
$$

Here $M(s) X$ in Eq. (29) reflects the nominal linear optics, with the horizontal and vertical focusing strengths $k_{x}(s)$ and $k_{y}(x)$ from the external magnetic fields satisfying $k_{x}^{2}(s)=\kappa_{0}^{2}(s)-\kappa_{1}(s) \quad$ and $\quad k_{y}^{2}(s)=\kappa_{1}(s)$. The term $F^{[f]}(X, s)$ contains the normalized effective CSR forces as expressed in terms of potentials:

$$
\begin{aligned}
\tilde{F}_{x}(X, s) \simeq & \left(1+\kappa_{0} x\right)\left[\kappa_{0}\left(\tilde{A}_{s}-\beta_{0} \tilde{\Phi}-\frac{\tilde{\Phi}}{\gamma_{0}^{2}}\right)\right. \\
& +\left(1+\kappa_{0} x\right)\left(\frac{\partial\left(\tilde{A}_{s}-\beta_{0} \tilde{\Phi}\right)}{\partial x}-\frac{1}{\gamma_{0}^{2}} \frac{\partial \tilde{\Phi}}{\partial x}\right) \\
& \left.+y^{\prime}\left(\frac{\partial \tilde{A}_{y}}{\partial x}-\frac{\partial \tilde{A}_{x}}{\partial y}\right)-\left(\frac{\partial \tilde{A}_{x}}{\partial s}-\kappa_{0} x \frac{\partial \tilde{A}_{x}}{\partial z}\right)\right]
\end{aligned}
$$

$$
\begin{aligned}
\tilde{F}_{y}(X, s) \simeq & \left(1+\kappa_{0} x\right)\left[\left(1+\kappa_{0} x\right)\left(\frac{\partial\left(\tilde{A}_{s}-\beta_{0} \tilde{\Phi}\right)}{\partial y}-\frac{1}{\gamma_{0}^{2}} \frac{\partial \tilde{\Phi}}{\partial y}\right)\right. \\
& \left.+x^{\prime}\left(\frac{\partial \tilde{A}_{x}}{\partial y}-\frac{\partial \tilde{A}_{y}}{\partial x}\right)-\left(\frac{\partial \tilde{A}_{y}}{\partial s}-\kappa_{0} x \frac{\partial \tilde{A}_{y}}{\partial z}\right)\right], \\
\tilde{F}_{H}(X, s) \simeq & \left(1+\kappa_{0} x\right)\left(\frac{\partial\left(\tilde{A}_{s}-\beta_{0} \tilde{\Phi}\right)}{\partial z}-\frac{1}{\gamma_{0}^{2}} \frac{\partial \tilde{\Phi}}{\partial z}\right) \\
& +x^{\prime} \frac{\partial \tilde{A}_{x}}{\partial z}+y^{\prime} \frac{\partial \tilde{A}_{y}}{\partial z} .
\end{aligned}
$$

Note that for $U=\tilde{A}_{x}$ or $\tilde{A}_{y}$, the expansion

$$
\frac{d U}{d s}=x^{\prime} \frac{\partial U}{\partial x}+y^{\prime} \frac{\partial U}{\partial y}+z^{\prime} \frac{\partial U}{\partial z}+\frac{\partial U}{\partial s}
$$

is used in deriving Eqs. (29)-(33) from Eqs. (19)-(24). In Sec. VI, we will focus on the analysis of the dominant term in $\tilde{F}_{H}(X, s)$ of Eq. (33).

Let $f(X, s)$ denote the phase-space distribution function of the bunch. The superscript $[f]$ of $F^{[f]}$ in Eq. (29) shows the functional dependence of the effective CSR forces on $f(X, s)$ through the dependence of the scalar and vector potentials on the charge phase-space distribution. Let $N$ be the total number of electrons in the bunch; then for $d X=$ $d x d x^{\prime} d y d y^{\prime} d z d \delta_{H}$, the integral over the infinite phasespace volume is

$$
\int f(X, s) d X=N
$$

Instead of $f_{0}(\zeta)$ with $\zeta=[\mathbf{r}(0), \dot{\mathbf{r}}(0)]$ in Eq. (4), here we use the initial bunch phase-space distribution $f_{0}(\zeta)$ with $\zeta=\left.X\right|_{s=0}$. The particle number conservation then requires $f_{0}(\zeta) d \zeta=f(X, s) d X$ for an infinitesimal initial phasespace volume $d \zeta$. This enables us to write the collective interaction potentials in Eq. (4) on a test particle in terms of $f(X, s)$. Using Eqs. (4), (8), and (15), and changing variable from $t_{r}$ to $s_{r}$ via $t_{r}=\left[s_{r}-z\left(s_{r}, \zeta\right)\right] / \beta_{0} c$, with

$$
\begin{aligned}
\frac{d r^{\mu}\left(s_{r}\right)}{d s_{r}}= & \left(\frac{1+\kappa_{0}\left(s_{r}\right) x_{r}}{\beta_{0}},\left[1+\kappa_{0}\left(s_{r}\right) x_{r}\right] \mathbf{e}_{s}\left(s_{r}\right)\right. \\
& \left.+x_{r}^{\prime} \mathbf{e}_{x}\left(s_{r}\right)+y_{r}^{\prime} \mathbf{e}_{y}\left(s_{r}\right)\right)
\end{aligned}
$$

we have

$$
\begin{aligned}
\tilde{\Phi}(x, y, z, s)= & \frac{2 \beta_{0} r_{e}}{\gamma_{0}} \int d s_{r} \int d X_{r} f\left(X_{r}, s_{r}\right)\left[1+\kappa_{0}\left(s_{r}\right) x_{r}\right] \times \theta\left[\left(s-s_{r}\right)-\left(z-z_{r}\right)\right] \\
& \times \delta\left\{\left[\left(s-s_{r}\right)-\left(z-z_{r}\right)\right]^{2}-\beta_{0}^{2}\left[\mathbf{r}(x, y, s)-\mathbf{r}\left(x_{r}, y_{r}, s_{r}\right)\right]^{2}\right\}, \\
\tilde{\mathbf{A}}(x, y, z, s)= & \frac{2 \beta_{0}^{2} r_{e}}{\gamma_{0}} \int d s_{r} \int d X_{r} f\left(X_{r}, s_{r}\right)\left\{\left[1+\kappa_{0}\left(s_{r}\right) x_{r}\right] \mathbf{e}_{s}\left(s_{r}\right)+x_{r}^{\prime} \mathbf{e}_{x}\left(s_{r}\right)+y_{r}^{\prime} \mathbf{e}_{y}\left(s_{r}\right)\right\} \\
& \times \theta\left[\left(s-s_{r}\right)-\left(z-z_{r}\right)\right] \delta\left\{\left[\left(s-s_{r}\right)-\left(z-z_{r}\right)\right]^{2}-\beta_{0}^{2}\left[\mathbf{r}(x, y, s)-\mathbf{r}\left(x_{r}, y_{r}, s_{r}\right)\right]^{2}\right\},
\end{aligned}
$$

with $r_{e}$ the classical electron radius, $X_{r}=\left(x_{r}, x_{r}^{\prime}, y_{r}, y_{r}^{\prime}, z_{r}, \delta_{H r}\right)$ representing the source particle's phase-space variables at the retarded path length $s_{r}$, and $\mathbf{r}(x, y, s)$ and $\mathbf{r}\left(x_{r}, y_{r}, s_{r}\right)$ the position vectors [see Eq. (5)] for the test and source particles, respectively. In Eq. (37), the Dirac-delta function and the Heaviside step function together imply that the source particle's 
retarded longitudinal position is determined by

$$
\begin{aligned}
z_{r} & =z_{r}\left(x, y, z, s ; x_{r}, y_{r}, s_{r}\right) \\
& \equiv z-s+s_{r}+\beta_{0}\left|\mathbf{r}(x, y, s)-\mathbf{r}\left(x_{r}, y_{r}, s_{r}\right)\right|,
\end{aligned}
$$

which imposes the retardation relation

$$
t_{r}=t-\frac{\left|\mathbf{r}(x, y, s)-\mathbf{r}\left(x_{r}, y_{r}, s_{r}\right)\right|}{c}
$$

on the interaction between particles.

Another familiar expression for the potentials, with $X_{r}^{\perp}=\left(x_{r}, x_{r}^{\prime}, y_{r}, y_{r}^{\prime}\right)$, is

$$
\begin{aligned}
& \tilde{\Phi}(x, y, z, s)=\frac{r_{e}}{\gamma_{0}} \int d s_{r} \int d X_{r}^{\perp} d \delta_{H r}\left[1+\kappa_{0}\left(s_{r}\right) x_{r}\right] \frac{f\left[X_{r}^{\perp}, z_{r}\left(x, y, z, s ; x_{r}, y_{r}, s_{r}\right), \delta_{H r}, s_{r}\right]}{\left|\mathbf{r}(x, y, s)-\mathbf{r}\left(x_{r}, y_{r}, s_{r}\right)\right|}, \\
& \tilde{\mathbf{A}}(x, y, z, s)=\frac{\beta_{0} r_{e}}{\gamma_{0}} \int d s_{r} \int d X_{r}^{\perp} d \delta_{H r}\left[\left(1+\kappa_{0}\left(s_{r}\right) x_{r}\right) \mathbf{e}_{s}\left(s_{r}\right)+x_{r}^{\prime} \mathbf{e}_{x}\left(s_{r}\right)+y_{r}^{\prime} \mathbf{e}_{y}\left(s_{r}\right)\right] \frac{f\left[X_{r}^{\perp}, z_{r}\left(x, y, z, s ; x_{r}, y_{r}, s_{r}\right), \delta_{H r}, s_{r}\right]}{\left|\mathbf{r}(x, y, s)-\mathbf{r}\left(x_{r}, y_{r}, s_{r}\right)\right|} .
\end{aligned}
$$

Here the potentials depend on $z$ only through $z_{r}\left(x, y, z, s, x_{r}, y_{r}, s_{r}\right)$, which makes the study of the longitudinal effective forces in Sec. VI easier.

Note that, for a particle with kinetic energy $E=(1+$ $\left.\delta_{E}\right) E_{0}$, using $H=E+e \Phi$ and Eq. (14), one has

$$
\delta_{H}=\delta_{E}+\tilde{\Phi}-\frac{1}{2 \gamma_{0}^{2}} .
$$

The reasons for choosing the special combination $X=$ $\left(x, x^{\prime}, y, y^{\prime}, z, \delta_{H}\right)$ as the dynamical variables in this paper are: (1) for vanishing effective CSR force $F^{[f]}$, we restore the nominal design optics from Eq. (29); (2) the phasespace distribution $f(X, s)$ can be directly related to many of the quantities measurable in the laboratory (see Appendix B 4); and (3) this set of phase-space parameters allows the cancellation effect to be explicitly manifested in the equations of motion, as can be seen in the first term of $\tilde{F}_{x}$ in Eq. (31) (see Refs. [12,16,17] for more details).

\section{THE EVOLUTION OF PHASE-SPACE DISTRIBUTION}

Based on the dynamical equations formulated in Sec. II, we can set up the evolution equation of the bunch phasespace distribution for our phase-space coordinates $\left(x, x^{\prime}, y, y^{\prime}, z, \delta_{H}\right)$. This is done in Appendix B 1, which gives the Vlasov equation

$$
\frac{\partial f}{\partial s}+\sum_{i=1}^{6} Y_{i}(X, s) \frac{\partial f}{\partial X_{i}}=0
$$

for $Y(X, s)$ defined in Eq. (29). As further shown in Appendix B 1, with the linear-optics approximation, one can write the integral form of Eq. (42) as

$$
f(X, s) \simeq f^{(0)}(X, s)-\int_{0}^{s} d s_{\tau}\left[F^{[f]}\left(X_{\tau}, s_{\tau}\right)\right]_{i} \frac{\partial f\left(X_{\tau}, s_{\tau}\right)}{\partial\left(X_{\tau}\right)_{i}},
$$

where

$$
f^{(0)}(X, s)=\rho\left[X_{0}=\mathcal{R}^{-1}(s) X, 0\right]
$$

for $\rho\left(X_{0}, 0\right)$ being the initial particle phase-space distribution for the normalized phase-space variables $X_{0}$, as introduced in Appendix A, $\mathcal{R}$ is the optics transport matrix given in Eq. (A5), and $F^{[f]}\left(X_{\tau}, s_{\tau}\right)$ is the effective CSR forces given in Eq. (30). Equation (43) manifests explicitly how the bunch phase-space distribution is perturbed by the effective CSR forces. For small effective CSR forces, the perturbation on the nominal phase-space distribution is yielded from the first iteration of Eq. (43):

$f(X, s) \simeq f^{(0)}(X, s)-\int_{0}^{s} d s_{\tau}\left[F^{\left[f^{(0)}\right]}\left(X_{\tau}, s_{\tau}\right)\right]_{i} \frac{\partial f^{(0)}\left(X_{\tau}, s_{\tau}\right)}{\partial\left(X_{\tau}\right)_{i}}$,

where the effective CSR force $F^{\left[f^{(0)}\right]}\left(X_{\tau}, s_{\tau}\right)$ is obtained from Eqs. (31)-(33) and (37) under the unperturbed-source approximation [18]. This approximation implies that the CSR force is calculated assuming that the initial phasespace distribution is transported through magnetic bends under nominal optical transport without being influenced by CSR interaction.

The evolution of a small longitudinal density perturbation on a stable distribution can be found in Appendix B 3, and the relations of the phase-space distribution $f(X, s)$ to the physical quantities observed in the laboratory are listed in Appendix B 4.

\section{RETARDED POTENTIALS FOR A 2D ENERGY- CHIRPED BUNCH ON A CIRCULAR ORBIT}

In the previous sections, the equations of motion and the phase-space evolution equation are laid out for a charge distribution being transported along a curved orbit under an external EM field, and the perturbation of the charge phasespace distribution is expressed in terms of the effective CSR forces, or the curvature-induced bunch collective interaction forces. Here we are interested in studying the effect of bunch tilting ( $x-z$ correlation) on the effective CSR forces when an energy-chirped bunch $(\delta-z$ correlation) goes through a dispersive region $(x-\delta$ correlation) for 
processes such as bunch compression in a magnetic chicane.

Our first step is to analyze the retarded potentials for an energy-chirped bunch under the unperturbed-source approximation. This result will then be used in the following section for the analysis of $F^{\left[f^{(0)}\right]}$ in Eq. (45), which was obtained when one takes the first-order iteration in solving Eq. (43). Here the unperturbed-source approximation implies

$$
f\left(X_{r}, s_{r}\right)=f^{(0)}\left(X_{r}, s_{r}\right)=\rho\left[\mathcal{R}^{-1}\left(s_{r}\right) X_{r}, 0\right]=\rho\left(X_{r 0}, 0\right),
$$

where $X_{r}$ and $s_{r}$ are, respectively, the phase-space variables and the path length parameter for the retarded distribution $f\left(X_{r}, s_{r}\right)$ in Eq. (37), and $X_{r 0}=\mathcal{R}^{-1}\left(s_{r}\right) X_{r}$. The effect of energy chirping is included in the parameter $u$ in $\mathcal{R}(s)$ of Eq. (A5). For simplicity, in this paper we consider only a two-dimensional charge distribution in the $y=0$ plane, with $X=\left(x, x^{\prime}, 0,0, z, \delta_{H}\right)^{T}$ and $X_{r}=\left(x_{r}, x_{r}^{\prime}\right.$, $\left.0,0, z_{r}, \delta_{H r}\right)^{T}$. With the change of variables from $X_{r}$ to $X_{r 0}$,

$$
X_{r}=\left[x_{r}, x_{r}^{\prime}, 0,0, z_{r},\left(\delta_{H}\right)_{r}\right]^{T}=\mathcal{R}\left(s_{r}\right) X_{r 0},
$$

and using Eq. (46), the collective potentials in Eq. (37) become

$$
\begin{aligned}
& \tilde{\Phi}(x, z, s)=\frac{2 \beta_{0} r_{e}}{\gamma_{0}} \int d s_{r} \int d X_{r 0} \rho\left(X_{r 0}, 0\right) w_{0}\left(X_{r 0}, s_{r}\right) \delta(P) \theta(Q), \\
& \tilde{\mathbf{A}}(x, z, s)=\frac{2 \beta_{0}^{2} r_{e}}{\gamma_{0}} \int d s_{r} \int d X_{r 0} \rho\left(X_{r 0}, 0\right) \mathbf{w}\left(X_{r 0}, s_{r}\right) \delta(P) \theta(Q),
\end{aligned}
$$

where

$$
\left\{\begin{array}{l}
w_{0}\left(X_{r 0}, s_{r}\right)=1+\kappa_{0}\left(s_{r}\right) x_{r}\left(X_{r 0}, s_{r}\right) \\
\mathbf{w}\left(X_{r 0}, s_{r}\right)=\left[1+\kappa_{0}\left(s_{r}\right) x_{r}\left(X_{r 0}, s_{r}\right)\right] \mathbf{e}_{s}\left(s_{r}\right)+x_{r}^{\prime}\left(X_{r 0}, s_{r}\right) \mathbf{e}_{x}\left(s_{r}\right)
\end{array}\right.
$$

and $P, Q$ in Eq. (48) are

$$
\left\{\begin{array}{l}
P\left(x, z, s, X_{r 0}, s_{r}\right)=\left\{\left(s-s_{r}\right)-\left[z-z_{r}\left(X_{r 0}, s_{r}\right)\right]\right\}^{2}-\beta_{0}^{2}\left|\mathbf{r}(x, s)-\mathbf{r}\left[x_{r}\left(X_{r 0}, s_{r}\right), s_{r}\right]\right|^{2}, \\
Q\left(x, z, s, X_{r 0}, s_{r}\right)=\left(s-s_{r}\right)-\left[z-z_{r}\left(X_{r 0}, s_{r}\right)\right] .
\end{array}\right.
$$

In Eq. (48), $\delta(P)$ may contain both retarded and advanced solutions, and $\theta(Q)$ ensures the selection of only the retarded solution. For interactions within a single magnetic bend when both $s$ and $s_{r}$ are on the same circular orbit with radius $R_{0}=1 / \kappa_{0}$, one has $\left|\mathbf{r}(x, s)-\mathbf{r}\left(x_{r}, s_{r}\right)\right|^{2}$ in Eq. (50) as

$$
\begin{aligned}
\left|\mathbf{r}(x, s)-\mathbf{r}\left(x_{r}, s_{r}\right)\right|^{2}= & \left(1+\frac{x}{R_{0}}\right)\left(1+\frac{x_{r}}{R_{0}}\right) \\
& \times\left(2 R_{0} \sin \frac{s-s_{r}}{2 R_{0}}\right)^{2}+\left(x-x_{r}\right)^{2} .
\end{aligned}
$$

Because of the initial linear energy chirp, as the bunch is transported through a dispersive region, it will develop a horizontal-longitudinal correlation in the configuration space. This bunch tilt can be quantified as follows. Using Eq. (47) for the source particle, with $\mathcal{R}_{i j}\left(s_{r}\right)$ given in Eq. (A5), we write

$$
\begin{aligned}
& z_{r}\left(X_{r 0}, s_{r}\right)=z_{r 1}+\mathcal{R}_{55}\left(s_{r}\right) z_{r 0}, \\
& x_{r}\left(X_{r 0}, s_{r}\right)=x_{r 1}+\mathcal{R}_{15}\left(s_{r}\right) z_{r 0}
\end{aligned}
$$

with

$$
\begin{gathered}
z_{r 1}=\mathcal{R}_{5 j}\left(s_{r}\right)\left(Z_{r 0}\right)_{j}, \quad x_{r 1}=\mathcal{R}_{1 j}\left(s_{r}\right)\left(Z_{r 0}\right)_{j} \\
(j=1 \text { to } 6)
\end{gathered}
$$

for $Z_{r 0}$ representing the intrinsic transverse and energy offsets:

$$
Z_{r 0}=\left[x_{r 0}, x_{r 0}^{\prime}, 0,0,0,\left(\delta_{H}\right)_{r 0}\right]^{T} .
$$

Similarly, we write the coordinates for the test particle as

$$
z\left(X_{0}, s\right)=z_{1}+\mathcal{R}_{55}(s) z_{0}, \quad x\left(X_{0}, s\right)=x_{1}+\mathcal{R}_{15}(s) z_{0}
$$

with

$$
\begin{gathered}
z_{1}=\mathcal{R}_{5 j}(s)\left(Z_{0}\right)_{j}, \quad x_{1}=\mathcal{R}_{1 j}(s)\left(Z_{0}\right)_{j} \\
(j=1 \text { to } 6)
\end{gathered}
$$

for

$$
Z_{0}=\left[x_{0}, x_{0}^{\prime}, 0,0,0,\left(\delta_{H}\right)_{0}\right]^{T} .
$$

We then denote the bunch tilting factor as the $x-z$ slope at $s$ for a linear bunch with zero initial transverse emittance and uncorrelated energy spread, 


$$
\begin{aligned}
\xi(s) & =\left.\frac{x(s)}{z(s)}\right|_{x_{0}=x_{0}^{\prime}=\delta_{H 0}=0}=\frac{\mathcal{R}_{15}(s)}{\mathcal{R}_{55}(s)}=\frac{u R_{16}(s)}{1+u R_{56}(s)}, \\
\xi_{r} & =\xi\left(s_{r}\right) .
\end{aligned}
$$

For a nontilted bunch studied earlier [5-7], the bunch has zero energy chirp, $u=0$, and the tilting factor $\xi(s)=0$ for all $s$. For a thick bunch with nonvanishing intrinsic spread, the $x-z$ correlation will be different from that given in Eq. (58).

For the convenience of the following study, let us define

$$
\begin{aligned}
& \Delta s=s-s_{r}, \quad \Delta z=z-\mathcal{R}_{55}\left(s_{r}\right) z_{r 0}, \\
& \text { and } \quad(\bar{z}, \Delta \bar{z}, \Delta \bar{s})=\frac{1}{\left|R_{0}\right|}(z, \Delta z, \Delta s)
\end{aligned}
$$

for a constant bend radius $R_{0}=1 / \kappa_{0}$. We also define $\hat{x}$ and $\hat{\xi}$ as

$$
\begin{gathered}
\hat{x}_{r}=\frac{x_{r}}{R_{0}}, \quad \hat{x}=\frac{x}{R_{0}}, \\
\hat{\xi}_{r}(s)=\xi\left(s_{r}\right) \frac{R_{0}}{\left|R_{0}\right|}, \quad \hat{\xi}(s)=\xi(s) \frac{R_{0}}{\left|R_{0}\right|},
\end{gathered}
$$

which are invariant when the beam line is under mirror reflection $R_{0} \rightarrow-R_{0}$ and $x \rightarrow-x$. Note that, unlike the scaling by $\left|R_{0}\right|$ in Eq. (59), the sign of $R_{0}$ appears in Eq. (60). This is because bending systems, such as a bunch-compression chicane, often consist of bends with both positive and negative curvatures. The $x$ coordinates (of the Frenet frame) for the particle distribution, on the other hand, is defined for the whole beam line disregarding the variation of the curvature signs. Since the geometrical relation in Eq. (51) is relevant to $\left(R_{0}+x\right)$, one needs to give careful account of the relative signs of $x$ and $R_{0}$ when dealing with both positive and negative curvatures. The convention here is that $R_{0}>0$ if for positive $x$ we have $x+$
$R_{0}$ located on the outer radius from the center of the circular orbit.

For a test particle at $(x, z, s)$ to receive fields generated by source particles at $s_{r}$, knowing the source particles' intrinsic transverse and energy offset $Z_{r 0}$, we need to solve the initial longitudinal position $z_{r 0}$ of the source particles by evaluating $\delta(P)$ in Eq. (48). When both $s$ and $s_{r}$ are inside the same magnetic bend with radius $R_{0}$, using Eq. (51), $P$ in Eq. (50) becomes a quadratic function of $\Delta \bar{z}$

$$
P=R_{0}^{2}\left(a \Delta \bar{z}^{2}-2 b \Delta \bar{z}+c\right),
$$

with $a, b, c$ given in Appendix $\mathrm{C} 1$ in terms of $(x, z, s)$ and $\left(Z_{r 0}, s_{r}\right)$. Thus, we have for $b^{2}-a c \geq 0$

$$
\delta(P)=\frac{\delta\left(\Delta \bar{z}-\Delta \bar{z}^{(+)}\right)+\delta\left(\Delta \bar{z}-\Delta \bar{z}^{(-)}\right)}{2 R_{0}^{2} \sqrt{b^{2}-a c}},
$$

with $\Delta \bar{z}^{( \pm)}$the roots for $P=0$ :

$$
\Delta \bar{z}^{( \pm)}\left(x, z, s ; Z_{r 0}, s_{r}\right)=\frac{b \pm \sqrt{b^{2}-a c}}{a} .
$$

This leads us to the solution of $\bar{z}_{r 0}$

$$
\delta(P)=\frac{\delta\left(\bar{z}_{r 0}-\bar{z}_{r 0}^{(+)}\right)+\delta\left(\bar{z}_{r 0}-\bar{z}_{r 0}^{(-)}\right)}{2 R_{0}^{2}\left|\mathcal{R}_{55}\left(s_{r}\right)\right| \sqrt{b^{2}-a c}} \quad\left(b^{2}-a c>0\right),
$$

in which

$$
\bar{z}_{r 0}^{( \pm)}\left(x, z, s ; Z_{r 0}, s_{r}\right)=\frac{\bar{z}-\Delta \bar{z}^{( \pm)}}{\mathcal{R}_{55}\left(s_{r}\right)}=\frac{a \bar{z}-b \mp \sqrt{b^{2}-a c}}{a \mathcal{R}_{55}\left(s_{r}\right)} .
$$

The expression for $\bar{z}_{r 0}^{( \pm)}$can be obtained by substituting Eqs. (C1) and (C7) into Eq. (65):

$$
\bar{z}_{r 0}^{( \pm)}=\frac{-\Delta \bar{s}\left(1-\hat{\xi}_{r} \Delta \bar{s} / 2\right)-\left(\hat{\xi}_{r} \hat{x}-\bar{z}\right)-\left(\bar{z}_{r 1}-\hat{\xi}_{r} \hat{x}_{r 1}\right) \mp \sqrt{\omega_{0}+\omega_{1}}}{\left(1-\beta_{0}^{2} \hat{\xi}_{r}^{2}\right) \mathcal{R}_{55}\left(s_{r}\right)} .
$$

For the case of tilted bunch, with $\beta_{0}^{2} \xi_{r}^{2}>1$, it is convenient to use

$$
\hat{\chi}(s)=\frac{1}{\hat{\xi}(s)}, \quad \text { and } \quad \hat{\chi}_{r}=\frac{1}{\hat{\xi}_{r}} .
$$

With both the numerator and denominator multiplied by $\hat{\chi}_{r}$, Eq. (66) becomes

$$
\bar{z}_{r 0}^{( \pm)}=\frac{\Delta \bar{s}\left(\hat{\chi}_{r}-\Delta \bar{s} / 2\right)+\left(\hat{x}-\hat{\chi}_{r} \bar{z}\right)-\left(\hat{x}_{r 1}-\hat{\chi}_{r} \bar{z}_{r 1}\right) \pm \operatorname{sign}\left(\hat{\chi}_{r}\right) \sqrt{\left(\omega_{0}+\omega_{1}\right) \hat{\chi}_{r}^{2}}}{\left(\beta_{0}^{2}-\hat{\chi}_{r}^{2}\right) u \hat{R}_{16}\left(s_{r}\right)},
$$

with $\left(\omega_{0}+\omega_{1}\right) \hat{\chi}_{r}^{2}$ given by Eq. $(\mathrm{C} 10)$, and $\hat{R}_{16}\left(s_{r}\right)=R_{16}\left(s_{r}\right) \cdot \operatorname{sign}\left(\kappa_{0}\right)$.

In Sec. VI, we relabel the retardation solutions by taking away $\operatorname{sign}\left(\hat{\chi}_{r}\right)$ in Eq. (68):

$$
\bar{z}_{r 0}^{( \pm)}=\frac{\Delta \bar{s}\left(\hat{\chi}_{r}-\Delta \bar{s} / 2\right)+\left(\hat{x}-\hat{\chi}_{r} \bar{z}\right)-\left(\hat{x}_{r 1}-\hat{\chi}_{r} \bar{z}_{r 1}\right) \pm \sqrt{\left(\omega_{0}+\omega_{1}\right) \hat{\chi}_{r}^{2}}}{\left(\beta_{0}^{2}-\hat{\chi}_{r}^{2}\right) u \hat{R}_{16}\left(s_{r}\right)} .
$$


Compared to Eq. (68), the contributions to the CSR force from each of the solutions in Eq. (69) are continuous and easier for analysis.

For simplicity, in this paper, our study is limited only to the "single-bend" interaction. Here we define the "singlebend" interaction in such a way that for a test particle at path length $s$ in a magnetic bend, the CSR forces it receives from the rest of the bunch are all generated from the retarded path length inside the same magnetic bend as the one where the test particle resides. Namely, the contributions to the CSR forces originated from the region outside the "single bend" are negligible. This implies that the present study excludes the more complicated situation when both a straight path and a circular orbit are involved in the interaction. For a rigid-line bunch (with no bunch tilt) on a circular orbit, the "single-bend" interaction is equivalent to the "steady-state" interaction when the effective CSR forces on each particle remain unchanged along the path length. For an energy-chirped bunch in a bending system, however, the level of bunch tilt constantly changes as the result of varying dispersion; hence, the CSR interaction is always transient. Mathematically, the "single-bend" interaction implies that for $s_{1}<s_{r}<s_{2}$, with $s_{1}$ and $s_{2}$ the path lengths at the entrance and exit of the single bending magnet, the retarded solution falls within the bulk of the bunch initial longitudinal distribution, i.e.,

$$
-5 \sigma_{z 0} \leq z_{r 0}\left(x, z, s, Z_{r 0}, \Delta \bar{s}\right) \leq 5 \sigma_{z 0} \quad\left(s_{1}<s_{r}<s_{2}\right)
$$

with $\sigma_{z 0}$ the longitudinal rms bunch size at $s=0$.
We now assume that the initial normalized phase-space distribution $\rho\left(X_{r 0}, 0\right)$ takes the form

$$
\rho\left(X_{r 0}, 0\right)=N \rho_{\text {un }}\left(Z_{r 0}\right) \lambda_{0}\left(z_{r 0}\right)
$$

with $N$ the total number of electrons, $\lambda_{0}\left(z_{r 0}\right)$ the initial longitudinal charge-density distribution function and $\rho_{\text {un }}\left(Z_{r 0}\right)$ the initial intrinsic (or uncorrelated) transverse and energy distribution function, and

$$
\int \rho_{\mathrm{un}}\left(Z_{r 0}\right) d Z_{r 0}=1, \quad \text { and } \quad \int \lambda_{0}\left(z_{r 0}\right) d z_{r 0}=1 .
$$

The "single-bend" interaction implies that both $s_{r}$ and $s$ are on the same circular orbit, yielding

$$
\begin{aligned}
& \mathbf{e}_{s}\left(s_{r}\right) \cdot \mathbf{e}_{x}(s)=-\mathbf{e}_{x}\left(s_{r}\right) \cdot \mathbf{e}_{s}(s)=\sin \frac{s-s_{r}}{R_{0}} \\
& \mathbf{e}_{s}\left(s_{r}\right) \cdot \mathbf{e}_{s}(s)=\mathbf{e}_{x}\left(s_{r}\right) \cdot \mathbf{e}_{x}(s)=\cos \frac{s-s_{r}}{R_{0}}
\end{aligned}
$$

for our aforementioned convention on the sign of curvatures. Next we apply Eqs. (64) and (71) to Eq. (48), and let $d Z_{r 0}=d x_{r 0} d x_{r 0}^{\prime} d\left(\delta_{H}\right)_{r 0}$. Defining

$$
W\left(x, z, s ; Z_{r 0}, s_{r}\right)=\frac{\rho_{\mathrm{un}}\left(Z_{r 0}\right)}{\left|\mathcal{R}_{55}\left(s_{r}\right)\right| \sqrt{b^{2}-a c}},
$$

one obtains from Eq. (48) the potential terms used in Eqs. (31)-(33):

$$
\begin{aligned}
\tilde{\Phi}(x, z, s)= & \frac{\beta_{0} N r_{e}}{\gamma_{0}\left|R_{0}\right|}\left[\int_{\Omega^{(+)}} d s_{r} d Z_{r 0} W\left(x, z, s ; Z_{r 0}, s_{r}\right) \lambda\left(z_{r 0}^{(+)}\right) H_{0}\left(z_{r 0}^{(+)}, Z_{r 0}, s_{r}\right)\right. \\
& \left.+\int_{\Omega^{(-)}} d s_{r} d Z_{r 0} W\left(x, z, s ; Z_{r 0}, s_{r}\right) \lambda\left(z_{r 0}^{(-)}\right) H_{0}\left(z_{r 0}^{(-)}, Z_{r 0}, s_{r}\right)\right], \\
{\left[\tilde{A}_{s}-\beta_{0} \tilde{\Phi}\right]=} & \frac{\beta_{0}^{2} N r_{e}}{\gamma_{0}\left|R_{0}\right|}\left[\int_{\Omega^{(+)}} d s_{r} d Z_{r 0} W\left(x, z, s ; Z_{r 0}, s_{r}\right) \lambda\left(z_{r 0}^{(+)}\right) H_{s}\left(z_{r 0}^{(+)}, Z_{r 0}, s_{r}\right)\right. \\
& \left.+\int_{\Omega^{(-)}} d s_{r} d Z_{r 0} W\left(x, z, s ; Z_{r 0}, s_{r}\right) \lambda\left(z_{r 0}^{(-)}\right) H_{s}\left(z_{r 0}^{(-)}, Z_{r 0}, s_{r}\right)\right], \\
\tilde{A}_{x}(x, z, s)= & \frac{\beta_{0}^{2} N r_{e}}{\gamma_{0}\left|R_{0}\right|}\left[\int_{\Omega^{(+)}} d s_{r} d Z_{r 0} W\left(x, z, s ; Z_{r 0}, s_{r}\right) \lambda\left(z_{r 0}^{(+)}\right) H_{x}\left(z_{r 0}^{(+)}, Z_{r 0}, s_{r}\right)\right. \\
& \left.+\int_{\Omega^{(-)}} d s_{r} d Z_{r 0} W\left(x, z, s ; Z_{r 0}, s_{r}\right) \lambda\left(z_{r 0}^{(-)}\right) H_{x}\left(z_{r 0}^{(-)}, Z_{r 0}, s_{r}\right)\right],
\end{aligned}
$$

with $z_{r 0}^{( \pm)}=\left|R_{0}\right| \bar{z}_{r 0}^{( \pm)}$given in Eq. (65), and 


$$
\begin{aligned}
& H_{0}\left(z_{r 0}, Z_{r 0}, s_{r}\right)=1+\frac{\mathcal{R}_{15}\left(s_{r}\right) z_{r 0}+\mathcal{R}_{1 j}\left(s_{r}\right)\left(Z_{r 0}\right)_{j}}{R_{0}}, \\
& H_{s}\left(z_{r 0}, Z_{r 0}, s_{r}\right)=-\left[\mathcal{R}_{25}\left(s_{r}\right) z_{r 0}+\mathcal{R}_{2 j}\left(s_{r}\right)\left(Z_{r 0}\right){ }_{j}\right] \sin \frac{\Delta s}{R_{0}}-H_{0}\left(z_{r 0}, Z_{r 0}, s_{r}\right)(1-\cos \Delta \bar{s}), \\
& H_{x}\left(z_{r 0}, Z_{r 0}, s_{r}\right)=H_{0}\left(z_{r 0}, Z_{r 0}, s_{r}\right) \sin \frac{\Delta s}{R_{0}}+\left[\mathcal{R}_{25}\left(s_{r}\right) z_{r 0}+\mathcal{R}_{2 j}\left(s_{r}\right)\left(Z_{r 0}\right)_{j}\right] \cos \Delta \bar{s} .
\end{aligned}
$$

In Eq. (74), the ranges of phase-space integration, $\Omega^{( \pm)}$, are set to ensure the existence of solutions for Eq. (62) and to exclude the advanced solution:

$$
\begin{gathered}
\Omega^{( \pm)}:\left\{b^{2}-a c \geq 0 \quad\right. \text { and } \\
\left.Q^{( \pm)}=\Delta s+z_{r 1}-\Delta z^{( \pm)} \geq 0\right\},
\end{gathered}
$$

with $z_{r 1}$ and $\Delta z^{( \pm)}$given by Eqs. (53) and (63), respectively. Discussions of conditions in Eq. (76) can be found in Appendices C 2 and $\mathrm{C} 3$.

\section{RETARDATION SOLUTIONS FOR VARIOUS LEVELS OF BUNCH TILTING}

In Eq. (64) of Sec. IV, the retardation relation is solved for the CSR interaction on a circular orbit with curvature $\kappa_{0}$ and radius $R_{0}=1 / \kappa_{0}$, such that the initial longitudinal position of a source particle $z_{r 0}$ is expressed in terms of the bunch internal coordinates $(x, z, s)$ of the test particle, the initial intrinsic phase-space offset $Z_{r 0}$, and the retarded path length $s_{r}$ of the source particle. With $z_{0}$ in Eq. (55) denoting the initial internal longitudinal position of the test particle, and $\sigma_{z}(s)$ and $\sigma_{x}(s)$ the longitudinal and transverse rms bunch size at path length $s$ respectively, we can now list the retardation solutions for various levels of bunch tilt under the assumption of Eq. (C3).

\section{A. Nontilted bunch: $\boldsymbol{u}=\mathbf{0}$ or $\boldsymbol{\xi}_{\boldsymbol{r}}=\mathbf{0}$}

Setting $\xi_{r}=0$ and $\mathcal{R}_{55}\left(s_{r}\right)=1$ in Eq. (66), and assuming the thin-bunch condition

$$
\left(\hat{x}-\hat{x}_{r 1}\right)^{2} /(\Delta \bar{s})^{2} \ll \hat{x}+\hat{x}_{r 1}, \quad \text { or } \quad \frac{\sigma_{x}(s)}{\left|R_{0}\right|} \ll(\Delta \bar{s})^{2},
$$

we get the retardation solutions from Eq. (66) for $\Delta \bar{s}>0$ (back-front interaction),

$$
\bar{z}_{r 0}^{(-)} \simeq(\bar{z}+\hat{x} \Delta \bar{s} / 2)-\Delta \bar{s}^{3} / 24-\left(\bar{z}_{r 1}-\hat{x}_{r 1} \Delta \bar{s} / 2\right),
$$

and for $\Delta \bar{s}<0$ (front-back interaction)

$$
\begin{aligned}
\bar{z}_{r 0}^{(-)} \simeq & (\bar{z}+\hat{x}|\Delta \bar{s}| / 2)+2|\Delta \bar{s}|-|\Delta \bar{s}|^{3} / 24 \\
& -\left(\bar{z}_{r 1}-\hat{x}_{r 1}|\Delta \bar{s}| / 2\right) .
\end{aligned}
$$

For a bunch with the intrinsic horizontal emittance and uncorrelated (canonical) energy spread small enough to satisfy

$$
\hat{x}_{1} \sim \hat{x}_{r 1} \ll|\Delta \bar{s}|^{2}, \quad \text { and } \quad \bar{z}_{1} \sim \bar{z}_{r 1} \ll|\Delta \bar{s}|^{3},
$$

the solutions in Eqs. (78) and (79) reduce to

$$
\bar{z}_{0}-\bar{z}_{r 0}^{(-)} \simeq \Delta \bar{s}^{3} / 24 \quad(\Delta \bar{s}>0)
$$

and

$$
\bar{z}_{r 0}^{(-)}-\bar{z}_{0} \simeq 2|\Delta \bar{s}|-|\Delta \bar{s}|^{3} / 24 \quad(\Delta \bar{s}<0) .
$$

Here Eq. (81) indicates that, for a nontilted thin bunch, the back-front $\left(s_{r}<s\right)$ interaction corresponds to the tail-head solution, meaning that a test particle at a bunch head receives interaction generated by a source particle at the bunch tail, i.e., $z_{r 0}^{(-)}<z_{0}$. On the other hand, Eq. (82) indicates that the front-back $\left(s_{r}>s\right)$ interaction corresponds to the head-tail solution, implying that a test particle at the bunch tail receives interaction from a source particle at the bunch head, i.e., $z_{r 0}^{(-)}>z_{0}$. Note $\bar{z}_{r 0}^{(+)}$is excluded because $Q^{(+)}$in Eq. (76) is negative (see Appendix C 3 a).

\section{B. Small tilt}

Let us assume $O(\hat{x}) \ll(\Delta \bar{s})^{2}<1$ and consider a small tilt when $|a c| \ll b^{2}$. The retardation solutions can then be obtained by applying this small tilt condition to Eq. (66). For $\Delta \bar{s}>0$, we have a tail-head solution for the back-front interaction

$$
\bar{z}_{r 0}^{(-)} \simeq \frac{(\bar{z}+\hat{x} \Delta \bar{s} / 2)-\Delta \bar{s}^{3} / 24-\left(\bar{z}_{r 1}-\hat{x}_{r 1} \Delta \bar{s} / 2\right)}{\mathcal{R}_{55}\left(s_{r}\right)\left(1-\xi_{r} \Delta \bar{s} / 2\right)},
$$

which reduces to Eq. (78) when $u=0$. For $\xi_{r}^{2}<1$, the head-tail solution exists for the front-back $(\Delta \bar{s}<0)$ interaction

$$
\bar{z}_{r 0}^{(-)}=\frac{1}{\mathcal{R}_{55}\left(s_{r}\right)}\left[\bar{z}+\frac{2|\Delta \bar{s}|}{1-\xi_{r}^{2}}\left(1-\hat{\xi}_{r} \frac{\Delta \bar{s}}{2}\right)\right]+\cdots .
$$

This solution runs to infinity as $\xi_{r}^{2}$ approaches 1 . According to Appendix C 3 , as $\xi_{r}^{2}$ increases to a value bigger than 1, another head-tail solution appears for the back-front interaction. Namely, for $\xi_{r}^{2}>1$, a second solution exists for $\Delta \bar{s}>0$,

$$
\bar{z}_{r 0}^{(+)}=\frac{1}{\mathcal{R}_{55}\left(s_{r}\right)}\left[\bar{z}+\frac{2 \Delta \bar{s}}{\xi_{r}^{2}-1}\left(1-\hat{\xi}_{r} \frac{\Delta \bar{s}}{2}\right)\right]+\cdots
$$




\section{Moderate tilt}

Here we are mainly interested in the regime $\xi_{r}^{2} \gg 1$. Now instead of $\xi_{r}$, it is more convenient to use $\chi_{r}=1 / \xi_{r}$, with $\chi_{r}^{2} \ll 1$.

In Appendix C 2 and $\mathrm{C} 3$, for a test particle at $(x, z, s)$ to receive EM fields generated by a source particle at $s_{r}$, we solved the range of $Z_{r 0}$ for the existence of the retarded solution $z_{r 0}^{( \pm)}$. In particular, for $\eta$ defined in Eq. (C8), we found that $z_{r 0}^{( \pm)}$exists only when Eq. (C27) holds. Here we consider a special parameter regime for an undercompressed bunch, in which $\chi^{2}$ is large enough that $\eta_{\min }\left(x, z, s, s_{r}\right)$ in Eq. (C27) is approximately

$$
\eta_{\min }\left(x, z, s, s_{r}\right) \simeq \frac{\Delta \bar{s} \hat{\chi}^{2}}{2}
$$

and the main contributions to the CSR interaction on the particles are generated from $\Delta \bar{s}(>0)$ satisfying

$$
\sigma_{\eta} \ll \eta_{\min }\left(x, z, s, s_{r}\right)
$$

where $\sigma_{\eta}$ is the rms of $\eta$,

$$
\sigma_{\eta}\left(s_{r}\right)=\sqrt{\left[\alpha_{1}\left(s_{r}\right) \sigma_{x 0}\right]^{2}+\left[\alpha_{2}\left(s_{r}\right) \sigma_{x p 0}\right]^{2}+\left[\alpha_{6}\left(s_{r}\right) \sigma_{H 0}\right]^{2}}
$$

for $\alpha_{j}\left(s_{r}\right)$ given in Eq. (C9). Note Eq. (87) implies that the cutoff of the intrinsic phase space in Eq. (C27) happens beyond the bunch intrinsic spread and thus does not set an actual limit to the particle participation of the CSR interaction. We then have from Eqs. (C7) and (C13)

$$
\begin{aligned}
\left(\omega_{0}+\omega_{1}\right) \chi_{r}^{2} & \simeq \eta_{\min } \eta_{\max } \\
& \simeq \Delta \bar{s}^{2}\left[\left(\hat{\chi}_{r}-\frac{\Delta \bar{s}}{2}\right)^{2}+\frac{\Delta \bar{s}^{2}}{12}\right]>0 .
\end{aligned}
$$

Consequently, for $\Delta \bar{s}>0$, Eq. (69) reduces to

$$
\begin{aligned}
\bar{z}_{r 0}^{(-)} \simeq \frac{\left(\bar{z}+\hat{x} \frac{\Delta \bar{s}}{2}\right)-\left(\bar{z}_{r 1}-\hat{x}_{r 1} \frac{\Delta \bar{s}}{2}\right)-\frac{\Delta \bar{s}^{3}}{24}\left(1-\hat{\chi}_{r}^{2}\right)}{u \hat{R}_{16}\left(s_{r}\right)\left(1-\hat{\chi}_{r}^{2}\right)\left(\hat{\chi}_{r}-\frac{\Delta \bar{s}}{2}\right)}, \\
\bar{z}_{r 0}^{(+)} \simeq \frac{1}{u \hat{R}_{16}\left(s_{r}\right)\left(1-\hat{\chi}_{r}^{2}\right)\left(\hat{\chi}_{r}-\frac{\Delta \bar{s}}{2}\right)}\left\{2 \Delta \bar{s}\left(\hat{\chi}_{r}-\frac{\Delta \bar{s}}{2}\right)^{2}\right. \\
+\frac{\Delta \bar{s}^{3}}{24}\left(1-\hat{\chi}_{r}^{2}\right)+\left[\hat{x}\left(2 \hat{\chi}_{r}-\frac{3 \Delta \bar{s}}{2}\right)-\bar{z}\right] \\
\left.+\left[\bar{z}_{r 1}-\hat{x}_{r 1}\left(2 \hat{\chi}_{r}-\frac{\Delta \bar{s}}{2}\right)\right]\right\} .
\end{aligned}
$$

Furthermore, with Eq. (55) for $z$ and $x$, and results in Appendix D for $O\left[R_{0} \Delta \bar{s}^{2} / \hat{R}_{16}(s)\right] \ll 1$, the above retarded solutions become

$$
\begin{aligned}
\bar{z}_{r 0}^{(-)} \simeq \bar{z}_{0} & -\frac{1}{\mathcal{R}_{55}(s)[1+\Delta \bar{s} / 2 \hat{\chi}(s)]} \\
& \times\left[\frac{\Delta \bar{s}^{3}}{24}-\left(\bar{z}_{1}+\hat{x}_{1} \frac{\Delta \bar{s}}{2}\right)+\left(\bar{z}_{r 1}-\hat{x}_{r 1} \frac{\Delta \bar{s}}{2}\right)\right],
\end{aligned}
$$

$$
\begin{aligned}
\bar{z}_{r 0}^{(+)} \simeq & \bar{z}_{0}+\frac{1}{\mathcal{R}_{55}(s)[1+\Delta \bar{s} / 2 \hat{\chi}(s)]} \\
& \times\left\{\frac{\Delta \bar{s}^{3}}{24}+2 \Delta \bar{s}\left(\hat{\chi}_{r}-\frac{\Delta \bar{s}}{2}\right)^{2}-\left[\bar{z}_{1}-\hat{x}_{1}\left(2 \hat{\chi}+\frac{\Delta \bar{s}}{2}\right)\right]\right. \\
& \left.+\left[\bar{z}_{r 1}-\hat{x}_{r 1}\left(2 \hat{\chi}+\frac{3 \Delta \bar{s}}{2}\right)\right]\right\} .
\end{aligned}
$$

For small initial intrinsic spread satisfying Eq. (80), we have from Eqs. (92) and (93)

$$
\begin{aligned}
& \bar{z}_{0}-\bar{z}_{r 0}^{(-)} \simeq \frac{\Delta \bar{s}^{3} / 24}{\mathcal{R}_{55}(s)(1+\Delta \bar{s} / 2 \hat{\chi})}, \\
& \bar{z}_{r 0}^{(+)}-\bar{z}_{0} \simeq \frac{1}{\mathcal{R}_{55}(s)(1+\Delta \bar{s} / 2 \hat{\chi})} \\
& \times\left[2 \Delta \bar{s} \hat{\chi}^{2}\left(1+\frac{\Delta \bar{s}}{2 \hat{\chi}}\right)^{2}+\frac{\Delta \bar{s}^{3}}{24}\right] .
\end{aligned}
$$

Note that compared to the usual nontilted bunch case in Eq. (81), the tail-head interaction in Eq. (94) has an additional factor $(1+\Delta \bar{s} / 2 \hat{\chi})$. Unlike the nontilted case in Eq. (82), where the head-tail interaction is only generated at $s_{r}>s$, for a tilted line bunch with $\chi^{2}<\beta_{0}^{2}$, the head-tail interaction in Eq. (95) is generated at $s_{r}<s$. For an undercompressed bunch, as the tilt factor $\xi$ becomes larger, or $\chi$ smaller, $z_{r 0}^{(+)}-z_{0}$ in Eq. (95) gets smaller compared to its counterpart in Eq. (82). An illustration of the interaction of two source particles with a test particle on a circular orbit for a tilted bunch is shown in Fig. 1 (for $s_{r}<s$ ).

We now show that the condition in Eq. (87), under which one gets the retardation solutions in Eqs. (90) and (91), is satisfied only when the level of bunch tilt is under a certain constraint. Let the length of the characteristic structure in the initial bunch be $l_{z 0}$. Then for a thin bunch satisfying Eq. (80), we define the characteristic interaction angle

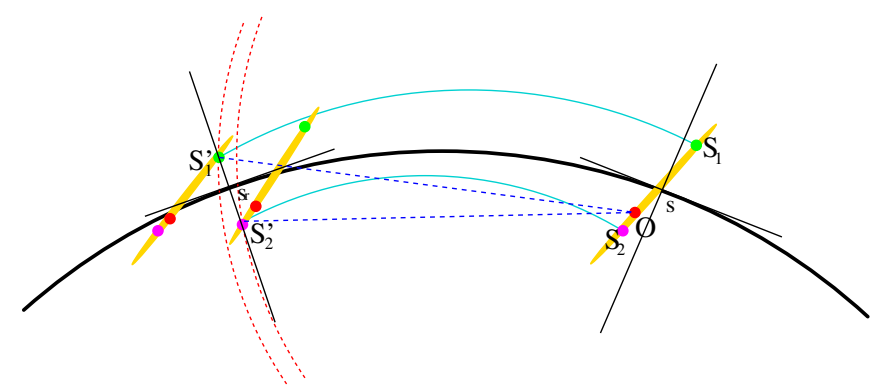

FIG. 1. (Color) Illustration of the interaction of the source particles $S_{1}^{\prime}$ and $S_{2}^{\prime}$ at path length $s_{r}$ with the test particle $O$ at path length $s$ for a tilted bunch. The retardation requires $\widehat{S_{1}^{\prime} S_{1}}=$ $\left(v_{1} / c\right) \overline{S_{1}^{\prime} O}$ and $\widehat{S_{2}^{\prime} S_{2}}=\left(v_{2} / c\right) \overline{S_{2}^{\prime} O}$ (may not be seen from drawing which is not to scale). The source particles $S_{1}^{\prime}$ and $S_{2}^{\prime}$ are at the intersections of $s=s_{r}$ with the past light cones (dashed red arcs) of the test particle $O$ at $(x, z, s)$, with the retarded velocities of $v_{1}$ and $v_{2}$, respectively. 


$$
\phi=\frac{\mathcal{R}_{55}(s) l_{z 0}}{\left|R_{0}\right|} .
$$

As can be seen from Eq. (94), the main contribution of $z_{r 0}^{(-)}$ (at $\Delta \bar{s}>0$ ) to the CSR force comes from the range $O\left(z_{0}-\right.$ $\left.z_{r 0}^{(-)}\right) \simeq l_{z 0}$, or

$$
O(\Delta \bar{s}) \sim(24 \phi)^{1 / 3} .
$$

Combining this range of $\Delta \bar{s}$ with Eq. (87), the condition for moderate tilt becomes

$$
\sigma_{\eta}\left(s_{r}\right) \ll \frac{\chi^{2}\left(s_{r}\right)}{2}(24 \phi)^{1 / 3} .
$$

Similarly, according to Eq. (95), the contribution of $z_{r 0}^{(+)}$at $\Delta \bar{s}>0$ to the CSR force arises from the range $O\left(z_{r 0}^{(+)}-\right.$ $\left.z_{0}\right) \simeq l_{z 0}$, or

$$
O(\Delta \bar{s}) \sim \frac{\phi}{\chi^{2}} .
$$

For a moderate tilt when $\chi \gg \phi^{1 / 3}$, the range of head-tail interaction in Eq. (99) is much smaller compared to that for tail-head in Eq. (97).

As for the front-back $(\Delta \bar{s}<0)$ interaction, from Eq. (C17), we find that the retardation solutions exist only when $\eta \leq \eta_{\min } \simeq-2|\Delta \bar{s}|$. For a Gaussian bunch, $|\eta| \leq 5 \sigma_{\eta}\left(s_{r}\right)$, the solutions exist only for near-neighbor interaction when

$$
O(\Delta \bar{s}) \sim O\left[\sigma_{\eta}\left(s_{r}\right)\right] .
$$

As discussed in Sec. VI, the contributions of $z_{r 0}^{( \pm)}$at $\Delta \bar{s}<0$ to the effective longitudinal CSR forces are negligible.

\section{Strong tilt}

When the bunch is strongly tilted, as in the case of crossing over the full compression point, we have $O\left(\hat{\chi}_{r}\right)$ so small that Eq. (98) does not hold. This occurs when the main contributions to the CSR force on the test particles come from retarded path length $s_{r}$ such that

$$
O\left[\sigma_{\eta}\left(s_{r}\right)\right] \sim \eta_{\min }(x, z, s, \Delta \bar{s}) .
$$

Here Eq. (101) implies that, for the source particles at $s_{r}$ to interact with a test particle at $(x, z, s)$, due to retardation, there is a cutoff, $\eta=\eta_{\min }(x, z, s, \Delta \bar{s})$, of the intrinsic phase space $\left(x_{r 0}, x_{r 0}^{\prime}, \delta_{H r 0}\right)$ for the source particles, and this cutoff falls right amid the bunch intrinsic spread. In this case, there is no simplification of Eq. (69) as given in Sec. VC. The full analysis is carried out in Appendix E.

\section{EFFECTIVE LONGITUDINAL CSR FORCE FOR A LINEAR ENERGY-CHIRPED GAUSSIAN BUNCH}

We now study the "single-bend" effective longitudinal CSR force for a tilted bunch with $\beta_{0}^{2} \xi_{r}^{2}>1$. For a $2 \mathrm{D}$ energy-chirped bunch on a circular orbit, the effective longitudinal CSR force can be obtained by applying $\tilde{A}_{s}-$ $\beta_{0} \tilde{\Phi}$ and $\tilde{A}_{x}$ in Eq. (40) to $\tilde{F}_{H}$ in Eq. (33):

$$
\begin{aligned}
\tilde{F}_{H}^{\left[f^{(0)}\right]}(X, s) & =\frac{\partial}{\partial z}\left[\left(1+\kappa_{0} x\right)\left(\tilde{A}_{s}-\beta_{0} \tilde{\Phi}\right)+x^{\prime} \tilde{A}_{x}-\frac{\Phi}{\gamma^{2}}\right] \\
& =\frac{\beta_{0} N r_{e}}{\gamma_{0}} \int d s_{r} d X_{r} W_{H}\left(X, s, X_{r}, s_{r}\right) \frac{\partial f\left(X_{r}, s_{r}\right)}{\partial z_{r}} \frac{\delta\left[\left(s-s_{r}\right)-\left(z-z_{r}\right)-\beta_{0}\left|\mathbf{r}(x, s)-\mathbf{r}\left(x_{r}, s_{r}\right)\right|\right]}{\left|\mathbf{r}(x, s)-\mathbf{r}\left(x_{r}, s_{r}\right)\right|},
\end{aligned}
$$

with

$$
\begin{aligned}
W_{H}\left[X, s, X_{r}, s_{r}\right]= & -(1+\hat{x})\left(1+\hat{x}_{r}\right) 2 \sin ^{2} \frac{\Delta \bar{s}}{2} \\
& -\left(\hat{x}_{r}^{\prime}-\hat{x}^{\prime}+\hat{x} \hat{x}_{r}^{\prime}-\hat{x}_{r} \hat{x}^{\prime}\right) \sin \Delta \bar{s} \\
& +\hat{x}^{\prime} \hat{x}_{r}^{\prime} \cos \Delta \bar{s}-\gamma^{-2}
\end{aligned}
$$

for

$\hat{x}_{r}^{\prime}=x_{r}^{\prime} \operatorname{sign}\left(\kappa_{0}\right)=\left[\mathcal{R}_{25}\left(s_{r}\right) z_{r 0}+\mathcal{R}_{2 j}\left(s_{r}\right)\left(Z_{r 0}\right){ }_{j}\right] \operatorname{sign}\left(\kappa_{0}\right)$,

$\hat{x}^{\prime}=x^{\prime} \operatorname{sign}\left(\kappa_{0}\right)=\left[\mathcal{R}_{25}(s) z_{0}+\mathcal{R}_{2 j}(s)\left(Z_{0}\right)_{j}\right] \operatorname{sign}\left(\kappa_{0}\right)$.

When $O\left(\hat{x}^{\prime}, \hat{x}_{r}^{\prime}\right) \ll O(\Delta \bar{s}) \ll 1$ and $O\left(\gamma^{-1}\right) \ll O(\Delta \bar{s}), W_{H}$ is dominated by its main term

$$
W_{H}\left[X, s, X_{r}, s_{r}\right] \simeq-(\Delta \bar{s})^{2} / 2 .
$$

Next, with the unperturbed-source approximation $f\left(X_{r}, s_{r}\right)=\rho\left[\mathcal{R}^{-1}\left(s_{r}\right) X_{r}, 0\right]$ and the change of variables
$X_{r 0}=\mathcal{R}^{-1}\left(s_{r}\right) X_{r}$, and $\left[\mathcal{R}^{-1}\left(s_{r}\right)\right]_{i 5}=(0,0,0,0,1,-u)^{T}$, we have in Eq. (102)

$$
\frac{\partial f\left(X_{r}, s_{r}\right)}{\partial z_{r}}=\frac{\partial \rho\left(X_{r 0}, 0\right)}{\partial z_{r 0}}-u \frac{\partial \rho_{0}\left(X_{r 0}\right)}{\partial\left(\delta_{H}\right)_{r 0}} .
$$

Moreover, with

$$
\begin{gathered}
\frac{\delta\left[\left(s-s_{r}\right)-\left(z-z_{r}\right)-\beta_{0}\left|\mathbf{r}(x, s)-\mathbf{r}\left(x_{r}, s_{r}\right)\right|\right]}{\beta_{0}\left|\mathbf{r}(x, s)-\mathbf{r}\left(x_{r}, s_{r}\right)\right|} \\
=2 \delta(P) \theta\left[\left(s-s_{r}\right)-\left(z-z_{r}\right)\right]
\end{gathered}
$$

for $\delta(P)$ given in Eq. (64), $Z_{r 0}$ in Eq. (54), and $\rho\left(X_{r 0}, 0\right)$ given in Eq. (71), Eq. (102) becomes 


$$
\begin{aligned}
\tilde{F}_{H}^{\left[f^{(0)}\right]}(X, s)= & \frac{\beta_{0}^{2} N r_{e}}{\gamma_{0}\left|R_{0}\right|}\left(\int_{\Omega^{(+)}} d s_{r} d Z_{r 0} g^{(+)}\right. \\
& \left.+\int_{\Omega^{(-)}} d s_{r} d Z_{r 0} g^{(-)}\right),
\end{aligned}
$$

where for $z_{r 0}^{( \pm)}=\left|R_{0}\right| \bar{z}_{r 0}^{( \pm)}$given by Eq. (69), the integrand $g^{( \pm)}$in Eq. (107) is

$$
\begin{aligned}
g^{( \pm)}= & g\left[X, s, Z_{r 0}, z_{r 0}^{( \pm)}\left(x, z, s, Z_{r 0}, s_{r}\right), s\right] \\
= & \frac{W_{H}\left[X, s, X_{r}\left(Z_{r 0}, z_{r 0}^{( \pm)}\right), s_{r}\right]}{\left|\mathcal{R}_{55}\left(s_{r}\right)\right| \sqrt{b^{2}-a c}} \\
& \times\left[\rho_{\text {un }}\left(Z_{r 0}\right) \frac{\partial \lambda_{0}\left(z_{r 0}^{( \pm)}\right)}{\partial z_{r 0}}-u \lambda_{0}\left(z_{r 0}^{( \pm)}\right) \frac{\partial \rho_{\text {un }}\left(Z_{r 0}\right)}{\partial\left(\delta_{H}\right)_{r 0}}\right] .
\end{aligned}
$$

In the following, as a simple example, we use Eqs. (107) and (108) to study the effective longitudinal force for a tilted bunch with a Gaussian initial phase-space distribution. In this case, the initial longitudinal density distribution in Eq. (71) is described by

$$
\lambda_{0}\left(z_{0}\right)=\frac{1}{\sqrt{2 \pi} \sigma_{z 0}} \exp \left(-\frac{\tilde{z}_{0}^{2}}{2}\right) \quad\left(\text { for } \tilde{z}_{0}=\frac{z_{0}}{\sigma_{z 0}}\right) .
$$

The intrinsic (or uncorrelated) distribution in $Z_{r 0}$ space, with transverse geometric emittance $\epsilon_{x 0}$ and (canonical) energy spread $\sigma_{H 0}$, is

$\rho_{\text {un }}\left(Z_{0}\right)=\frac{1}{2 \pi \epsilon_{x 0}} \exp \left(-\frac{\tilde{x}_{0}^{2}}{2}-\frac{\tilde{x}_{0}^{\prime 2}}{2}\right) \frac{1}{\sqrt{2 \pi} \sigma_{H 0}} \exp \left(-\frac{\tilde{\delta}_{H 0}^{2}}{2}\right)$,

where

$$
\tilde{x}_{0}=\frac{x_{0}}{\sigma_{x 0}}, \quad \tilde{x}_{0}^{\prime}=\frac{x_{0}^{\prime}}{\sigma_{x 0^{\prime}}}, \quad \tilde{\delta}_{H 0}=\frac{\delta_{H 0}}{\sigma_{H 0}}
$$

for $\sigma_{x 0}=\sqrt{\beta_{x 0} \epsilon_{x 0}}$ and $\sigma_{x 0^{\prime}}=\sqrt{\epsilon_{x 0} / \beta_{x 0}}$, with $\beta_{x 0}$ the initial transverse beta function at $s=0$. For a moderately tilted thin bunch, an analytical result for $\tilde{F}_{H}$ is presented in Sec. VI A. For general cases including thick bunches under strong compression, results of $\tilde{F}_{H}$ obtained via numerical integration are presented in Sec. VIB.

Note that in this section we focus on the $\tilde{F}_{H}$ arising from the back-front interaction, or $\Delta s>0$. This is because the interaction for $\Delta s<0$ takes place only for near-neighbor interaction, as indicated in Eq. (100). Thus, its contribution to the longitudinal effective force in Eq. (107) associated with the first term in Eq. (103) is largely suppressed by the $-\Delta \bar{s}^{2} / 2$ factor in Eq. (103).

\section{A. Analytical result for an undercompressed} $\left(\mathcal{R}_{55}(s)>0\right)$ thin bunch with moderate tilt

We now analyze the integral in Eq. (107) contributed from the main term of $W_{H}$ given by Eq. (104). Under the "single-bend" interaction in Eq. (70) and the moderately tilted-thin-bunch approximation in Eq. (98), we find that $\tilde{F}_{H}$ is dominated by the contribution from the tail-head interaction

$$
\begin{aligned}
\tilde{F}_{H} \simeq & \frac{\beta_{0}^{2} N r_{e}}{\gamma_{0}\left|R_{0}\right|} \int_{\Omega^{(-)}} d s_{r} d Z_{r 0} g\left[z_{r 0}=z_{r 0}^{(-)}\right] \\
\simeq & \frac{\beta_{0}^{2} N r_{e}}{2 \pi \gamma_{0}} \int_{0}^{\infty} \frac{d \Delta \bar{s}}{\left|\mathcal{R}_{55}(s)\right|} \frac{-\Delta \bar{s} / 2}{\sqrt{\Lambda(\Delta \bar{s}, \hat{\gamma})}} \\
& \times \int_{-\infty}^{\infty} d k \exp \left(-\frac{k^{2} \sigma_{z 0}^{2}}{2}\right) I_{A}(s, \Delta \bar{s}, k)
\end{aligned}
$$

for

$$
\begin{aligned}
I_{A}(s, \Delta \bar{s}, k)= & \int_{-\infty}^{\infty} d Z_{r 0}\left(i k+u \frac{\left(\tilde{\delta}_{H}\right)_{r 0}}{\sigma_{H 0}}\right) \rho_{\mathrm{un}}\left(Z_{r 0}\right) \\
& \times \exp \left[i k z_{r 0}^{(-)}\right]
\end{aligned}
$$

where

$$
\left|\mathcal{R}_{55}\left(s_{r}\right)\right| \sqrt{b^{2}-a c}|\simeq| \mathcal{R}_{55}(s) \mid \Delta \bar{s} \sqrt{\Lambda(\Delta \bar{s}, \hat{\gamma})}
$$

is used based on Eqs. (89) and (D5), $\tilde{z}_{r 0}^{(-)}$is given in Eq. (92), and

$$
\Lambda(\Delta \bar{s}, \hat{\chi})=\left(1+\frac{\Delta \bar{s}}{2 \hat{\chi}}\right)^{2}+\frac{(\Delta \bar{s})^{2}}{12 \hat{\chi}^{2}} .
$$

Here in Eq. (112), the upper integral limit for $\Delta \bar{s}$ is extended to infinity due to the "single-bend" approximation, and the lower integral limit for $\Delta \bar{s}$ is extended to $\Delta \bar{s}=0$. The latter approximation is justified for a thin bunch with moderate tilt, because when $\Delta \bar{s}$ is too small to satisfy Eq. (98), the small $\Delta \bar{s}$ contribution to $\tilde{F}_{H}$ in Eq. (112) is suppressed by the numerator in the integrand.

Next, let us define

$$
\Pi(s, \Delta \bar{s}, k)=\exp \left[-\frac{k^{2}}{2} \Sigma^{2}(s, \Delta \bar{s})\right]
$$

for

$$
\Sigma^{2}(s, \Delta \bar{s})=\sum_{j=1,2,6}\left(\frac{\mathcal{R}_{5 j}\left(s_{r}\right)-\hat{\mathcal{R}}_{1 j}\left(s_{r}\right) \Delta \bar{s} / 2}{\mathcal{R}_{55}(s)(1+\Delta \bar{s} / 2 \hat{\chi})}\right)^{2} \sigma_{0 j}^{2},
$$

where

$$
\sigma_{01}=\sigma_{x 0}, \quad \sigma_{02}=\sigma_{x 0^{\prime}}, \quad \sigma_{06}=\sigma_{H 0},
$$

and for $j=1,2,6$

$$
\hat{\mathcal{R}}_{1 j}\left(s_{r}\right)=\mathcal{R}_{1 j} \operatorname{sign}\left(\kappa_{0}\right) .
$$

With the identity 


$$
1-u \frac{\mathcal{R}_{56}\left(s_{r}\right)-\hat{\mathcal{R}}_{16}\left(s_{r}\right) \Delta \bar{s} / 2}{\mathcal{R}_{55}\left(s_{r}\right)\left(1-\Delta \bar{s} / 2 \hat{\chi}_{r}\right)}=\frac{1}{\mathcal{R}_{55}\left(s_{r}\right)\left(1-\Delta \bar{s} / 2 \hat{\chi}_{r}\right)},
$$

and Eq. (D4), $I_{A}$ in Eq. (112) becomes

$$
I_{A}(s, \Delta \bar{s}, k)=\frac{i k \Pi(s, \Delta \bar{s}, k) \exp \left[i k\left(z_{0}-\Delta z_{0}\right)\right]}{\mathcal{R}_{55}(s)(1+\Delta \bar{s} / 2 \hat{\chi})}
$$

for

$$
\Delta z_{0}=\frac{\left|R_{0}\right|}{\mathcal{R}_{55}\left(s_{r}\right)\left(1-\Delta \bar{s} / 2 \hat{\chi}_{r}\right)}\left[\frac{\Delta \bar{s}^{3}}{24}-\left(\bar{z}_{1}+\hat{x}_{1} \frac{\Delta \bar{s}}{2}\right)\right] .
$$

For an initial line bunch, with negligible initial intrinsic spreads satisfying

$$
\Sigma^{2}(s, \Delta \bar{s}) / \sigma_{z 0}^{2} \ll 1,
$$

and using Eq. (D4), we finally get

$$
\begin{aligned}
\tilde{F}_{H}= & \frac{\beta_{0}^{2} N r_{e}}{\sqrt{2 \pi} \gamma_{0}\left[\sigma_{z}(s)\right]} \\
& \times \int_{0}^{\infty} d \Delta \bar{s} \frac{\Delta \bar{s} / 2}{\mathcal{R}_{55}(s) \sqrt{\Lambda(s, \Delta \bar{s})}(1+\Delta \bar{s} / 2 \hat{\chi})} \\
& \times\left(\frac{z_{0}-\Delta z_{0}}{\sigma_{z 0}^{2}}\right) \exp \left[-\frac{\left(z_{0}-\Delta z_{0}\right)^{2}}{2 \sigma_{z 0}^{2}}\right]
\end{aligned}
$$

under the thin-bunch approximation

$$
\sigma_{x}(s) \simeq \mathcal{R}_{15}(s) \sigma_{z 0}, \quad \sigma_{z}(s) \simeq \mathcal{R}_{55}(s) \sigma_{z 0} .
$$

Let us define

$$
\alpha(s)=\hat{\xi}(s)\left[\bar{\sigma}_{z}(s)\right]^{1 / 3} \quad \text { for } \bar{\sigma}_{z}(s)=\frac{\sigma_{z}(s)}{\left|R_{0}\right|} .
$$

Furthermore, let $\Delta \tilde{s}$ and $\Delta \tilde{z}_{0}$ be

$$
\Delta \tilde{s}=\frac{\Delta \bar{s}}{\left[\bar{\sigma}_{z}(s)\right]^{1 / 3}} \quad \text { and } \quad \Delta \tilde{z}_{0}=\frac{\Delta \tilde{s}^{3}}{24(1+\alpha \Delta \tilde{s} / 2)} .
$$

Then for $\mathcal{R}_{55}(s)>0$, we obtain from Eq. (124) the line bunch result

$$
\tilde{F}_{H}\left(\tilde{z}_{0}, \alpha\right) \simeq \frac{2 N r_{e}}{\gamma_{0} 3^{1 / 3} \sqrt{2 \pi}\left[\sigma_{z}(s)\right]^{4 / 3}\left|R_{0}\right|^{2 / 3}} I\left(\tilde{z}_{0}, \alpha\right),
$$

with

$$
\begin{aligned}
I\left(\tilde{z}_{0}, \alpha\right)= & \frac{3^{1 / 3}}{4} \int_{0}^{\infty} d \Delta \tilde{s} \frac{\Delta \tilde{s}}{\Lambda_{1}(\Delta \tilde{s}, \alpha)}\left(\tilde{z}_{0}-\Delta \tilde{z}_{0}\right) \\
& \times \exp \left[-\frac{\left(\tilde{z}_{0}-\Delta \tilde{z}_{0}\right)^{2}}{2}\right]
\end{aligned}
$$

for

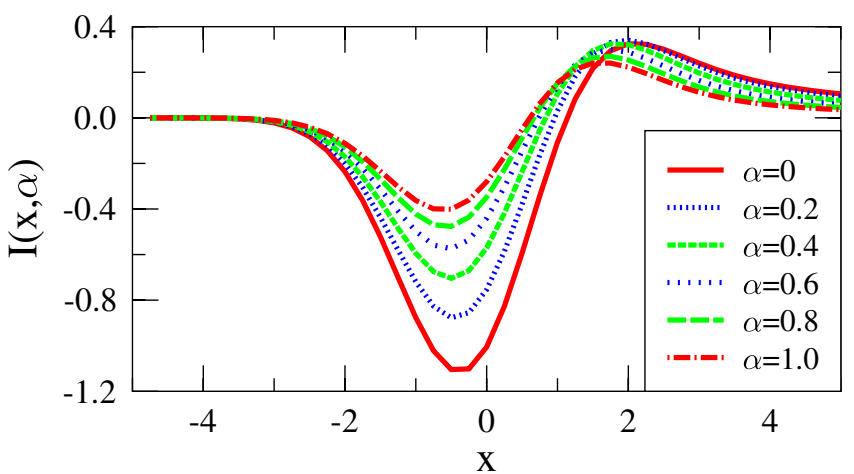

FIG. 2. (Color) $I(x, \alpha)$ vs $x$ for various $\alpha$ given by Eq. (129).

$$
\Lambda_{1}(\Delta \tilde{s}, \alpha)=\left(1+\frac{\alpha \Delta \tilde{s}}{2}\right) \sqrt{\left(1+\frac{\alpha \Delta \tilde{s}}{2}\right)^{2}+\frac{(\alpha \Delta \tilde{s})^{2}}{12}} .
$$

The behavior of $I\left(\tilde{z}_{0}, \alpha\right)$ is shown in Fig. 2, which agrees with the simulation results presented in Ref. [9]. Even though our study was carried out for a moderately tilted bunch with $\chi^{2} \ll 1$, we note that when $\alpha=0$ in Eqs. (127) and (128), we recover the results of the effective longitudinal CSR force for a nontilted bunch [8].

Note here that, for a thin bunch with negligible intrinsic spread, the absolute value of $\alpha(s)$ in Eq. (126) is exactly the parameter

$$
\alpha_{0}=\frac{\sigma_{x}}{\left(\sigma_{z}^{2} R_{0}\right)^{1 / 3}}
$$

used in Ref. [9] for the characterization of the effect of the bunch transverse size on the CSR interaction. This parameter reflects the comparison of the transverse bunch size $\sigma_{x}$ with the characteristic transverse length [8], $l_{x}=$ $2 /\left(9 \sigma_{z}^{2} R_{0}\right)^{1 / 3}$, for the CSR interaction. The analysis in this paper shows that the effective longitudinal CSR force is a function of $\alpha$ and $z$, as given by Eq. (128), only when the moderate-tilt condition in Eq. (98) is satisfied. This condition sets a constraint on the level of bunch tilt. The larger is the initial phase-space spread of the bunch, the tighter is the limit on the level of bunch tilt set by Eq. (98).

As mentioned earlier, the result in Eq. (128) is the contribution from the retardation solution $z_{r 0}^{(-)}$. The contribution to $\tilde{F}_{H}$ from the other retarded solution, $z_{r 0}^{(+)}$, is a factor $\alpha^{2}\left[\bar{\sigma}_{z}(s)\right]^{2 / 3}$ of that from $z_{r 0}^{(-)}$and is thus neglected here for $O\left\{\left[\bar{\sigma}_{z}(s)\right]^{1 / 3}\right\} \ll 1$. An example of comparison between the analytical result in Eq. (128), contributed from $z_{r 0}^{(-)}$only, and the full calculation from both $z_{r 0}^{(-)}$ and $z_{r 0}^{(+)}$is shown in Fig. 6 (at $s=1.1 \mathrm{~m}$ ). Details of the chicane used in Fig. 6 and the full calculation will be discussed in Sec. VIB. 


\section{B. Thick bunch around full compression-An example}

As discussed in Appendix $\mathrm{C}$, only source particles with phase-space parameters $Z_{r 0}=\left(x_{r 0}, x_{r 0}^{\prime}, \delta_{H r 0}\right)$ satisfying Eq. (C27) can generate EM fields at $s_{r}$ which reach the test particle at $(x, z, s)$. For the moderate-tilt regime, the bunch intrinsic phase-space spread is small enough and thus falls naturally within the boundary set by Eq. (C27) for the particles' participation in the CSR interaction at $s_{r}$. However, in the strong-tilt case given by Eq. (101), only a portion of the source particles' phase space $Z_{r 0}$ is selected by Eq. (C27) for the CSR interaction. In the evaluation of $\tilde{F}_{H}^{\left[f^{0}\right]}$ in Eq. (107), this selection is realized as a cutoff in the integral of $Z_{r 0}$. In Appendix E, this cutoff is treated by the change of variables, and the 4D integral in Eq. (107) is reduced to a $2 \mathrm{D}$ integral in Eq. (E36). To study $\tilde{F}_{H}$ for general bunch parameters, we numerically integrate the $2 \mathrm{D}$ integral in Eq. (E36) with the following steps. First, given a magnetic bending system, one gets the transport matrix $\mathcal{R}(s)$ and the tilt parameter $\chi(s)$. Then to calculate $\tilde{F}_{H}$ on a test particle at $(x, z, s)$, for each $s_{r}$ or $\Delta \bar{s}=\left(s-s_{r}\right) /\left|R_{0}\right|$, one finds $\left(\tilde{q}_{\min }, \tilde{q}_{\max }\right)$ as defined in Appendix E 2. The range of $\tilde{q}_{3}$ in the $2 \mathrm{D}$ integral is

$$
-10 \leq \tilde{q}_{3} \leq \min \left(\tilde{q}_{\min }, 10\right) \text {, }
$$

as dictated by $-10 \sigma_{q}\left(s_{r}\right)<q_{3}<10 \sigma_{q}\left(s_{r}\right)$ for the bunch intrinsic Gaussian phase-space distribution, and by $\tilde{q}_{3} \leq$ $\tilde{q}_{\text {min }}$ for retardation, with $\sigma_{q}\left(s_{r}\right)$ given in Eq. (E32). Consequently, the range for

$$
\tilde{p}=\sqrt{\left(\tilde{q}_{\min }-\tilde{q}_{3}\right)\left(\tilde{q}_{\max }-\tilde{q}_{3}\right)}
$$

is well defined, which allows one to integrate over $\tilde{p}$ for each $s_{r}$ in Eq. (E36). Next, we integrate over $\Delta \bar{s}$ in Eq. (E36) for a finite range of $\Delta \bar{s}$, beyond which the contribution to $\tilde{F}_{H}$ comes from source particles outside the longitudinal bunch distribution and is thus negligible.

In order to demonstrate the effect of bunch tilt on the CSR interaction, here we present our results of $\tilde{F}_{H}(x, z, s)$, as obtained from Eq. (E36) by numerical integration, in terms of

$$
G_{H}(\tilde{x}, \tilde{z}, s)=\frac{\tilde{F}_{H}\left[\tilde{x} \sigma_{x}(s), \tilde{z} \sigma_{z}(s), s\right]}{\tilde{F}_{H 0}},
$$

where the normalization factor $\tilde{F}_{H 0}(s)$ is related to the initial bunch length $\sigma_{z 0}$ at the entrance of the chicane by

$$
\tilde{F}_{H 0}=\frac{N r_{e}}{\gamma_{0} \sigma_{z 0}^{2}} \text {. }
$$

In Eq. (134), $(\tilde{x}, \tilde{z})$ are $(x, z)$ normalized by the bunch sizes at $s$

$$
\tilde{x}=\frac{x}{\sigma_{x}(s)}, \quad \tilde{z}=\frac{z}{\sigma_{z}(s)},
$$

where the bunch length $\sigma_{z}(s)$ is obtained from the projection of the actual tilted bunch on the design orbit

$$
\sigma_{z}(s)=\sqrt{\left[\sigma_{z}^{\text {un }}(s)\right]^{2}+\left[\mathcal{R}_{55}(s) \sigma_{z 0}\right]^{2}}
$$

with $\sigma_{z}^{\text {un }}(s)$ due to the initial uncorrelated spread

$$
\begin{aligned}
{\left[\sigma_{z}^{\mathrm{un}}(s)\right]^{2}=} & {\left[\mathcal{R}_{51}(s) \sigma_{x 0}\right]^{2}+\left[\mathcal{R}_{52}(s) \sigma_{x p 0}\right]^{2} } \\
& +\left[\mathcal{R}_{56}(s) \sigma_{H 0}\right]^{2},
\end{aligned}
$$

and similarly the horizontal bunch size $\sigma_{x}(s)$ is

$$
\sigma_{x}(s)=\sqrt{\left[\mathcal{R}_{11}(s) \sigma_{x 0}\right]^{2}+\left[\mathcal{R}_{12}(s) \sigma_{x p 0}\right]^{2}+\left[\mathcal{R}_{15}(s) \sigma_{z 0}\right]^{2}+\left[\mathcal{R}_{16}(s) \sigma_{H 0}\right]^{2}}
$$

To see the effect of the energy chirp on the effective longitudinal CSR force, we will compare our result of Eq. (134) with the equivalent result for a rigid-line bunch (abbreviated as r.l.b.)

$$
G_{H 0}=\frac{\tilde{F}_{H}^{\text {r.l.b. }}(\tilde{z}, s)}{\tilde{F}_{H 0}}
$$

for $[8]$

$$
\begin{aligned}
\tilde{F}_{H}^{\text {r.l.b. }}(\tilde{z}, s)= & \frac{2 N r_{e}}{\gamma_{0} 3^{1 / 3} \sqrt{2 \pi}\left[\sigma_{z}(s)\right]^{4 / 3}\left|R_{0}\right|^{2 / 3}} \\
& \times \int_{0}^{\infty} d \tilde{z}_{1} \frac{\tilde{z}-\tilde{z}_{1}}{\tilde{z}_{1}^{1 / 3}} e^{-\left(\tilde{z}-\tilde{z}_{1}\right)^{2} / 2}
\end{aligned}
$$

To illustrate the behavior of the effective longitudinal CSR force calculated from the above procedure, here we devise a model chicane as shown in Fig. 3. This chicane consists of three rectangular dipole magnets with bending radius $R=1 \mathrm{~m}$. The length for the two side dipoles is $L_{b}=0.3 \mathrm{~m}$ and the length for the center dipole is $L_{B}=$ $0.6 \mathrm{~m}$. The drift length between adjacent dipoles is $L_{d}=$ $0.4 \mathrm{~m}$. For the bunch entering the chicane, we choose an initial $\delta$ - $z$ correlation $u=-10.56 \mathrm{~m}^{-1}$. The resulting bunch-compression factor, $\mathcal{R}_{55}(s)=1+u R_{56}(s)$ vs $s$, is plotted in Fig. 4. Note that the bunch reaches full compression at $s_{c}=1.2 \mathrm{~m}$ when $\mathcal{R}_{55}\left(s_{c}\right)=0$ or $\chi\left(s_{c}\right)=0$. Here a long center bend, which ranges from $s=0.7$ to $1.3 \mathrm{~m}$, is

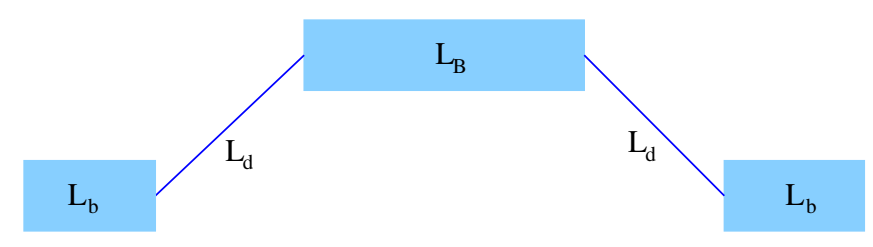

FIG. 3. (Color) Model chicane: $R=1 \mathrm{~m}, L_{b}=0.3 \mathrm{~m}, L_{B}=$ $0.6 \mathrm{~m}$, and $L_{d}=0.4 \mathrm{~m}$. 


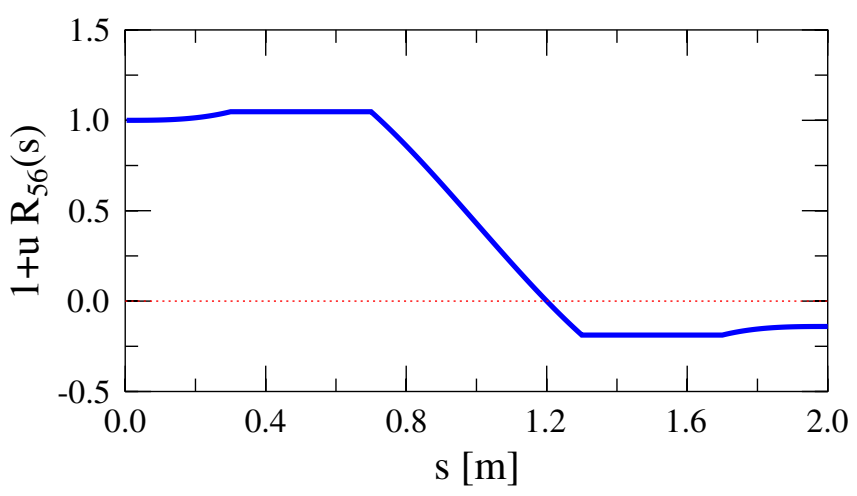

FIG. 4. (Color) Compression factor $\mathcal{R}_{55}(s)=1+u R_{56}(s)$ vs $s$ for the example chicane when $u=-10.56 \mathrm{~m}^{-1}$.

chosen for the chicane so that the "single-bendinteraction" approximation is valid in the end part of this bend. This allows us to study the behavior of the "singlebend" CSR force from $s=1.1 \mathrm{~m}$, when the bunch is undercompressed, to $s=1.3 \mathrm{~m}$, when the bunch is overcompressed. In this calculation, the horizontal betatron map in the chicane is approximated by that of a drift.

The bunch compression studied here, when $\mathcal{R}_{55}\left(s_{c}\right)=0$ at $s_{c}=1.2 \mathrm{~m}$ with nonzero values of $R_{16}\left(s_{c}\right)$ or $R_{26}\left(s_{c}\right)$, is an example of parasitic compression [19]. This is different from the critical compression case when the compression factor and the dispersion vanish simultaneously, or $\mathcal{R}_{55}\left(s_{c}\right)=0$ and $R_{16}\left(s_{c}\right)=R_{26}\left(s_{c}\right)$, which often takes place at the end of a bunch-compression chicane. Note that, due to nonzero $R_{51}\left(s_{c}\right)$ and $R_{52}\left(s_{c}\right)$, the minimum bunch length obtained at the parasitic compression is often longer than that obtained at the critical compression.

For the 2D Gaussian bunch being transported through the chicane, we studied the behavior of the longitudinal CSR force for two cases: one is the thin-bunch case with the normalized emittance $\epsilon_{n x 0}=1 \mathrm{~mm}-\mathrm{mrad}$ and the canonical energy spread $\sigma_{H 0}=10^{-4}$, and the another is the thick-bunch case with $\epsilon_{n x 0}=10 \mathrm{~mm}$-mrad and $\sigma_{H 0}=10^{-3}$. The common parameters for the two cases are the initial horizontal Twiss parameters $\beta_{x 0}=5 \mathrm{~m}$ and $\alpha_{x 0}=1$, and the initial bunch length and energy of the bunch: $\sigma_{z 0}=0.5 \mathrm{~mm}$ and $E_{0}=70 \mathrm{MeV}$. In the thinbunch case, for $s=1.1$ to $1.3 \mathrm{~m}$, the normalized effective longitudinal CSR force in Eq. (134) is displayed on the gray mesh of $(\tilde{x}, \tilde{z})$ around the bunch in Fig. 5. In addition, we use $N=1000$ particles to represent the Gaussian bunch distribution and propagate the particles through the chicane following the design optics. At each path length, the $\left(\tilde{x}_{k}, \tilde{z}_{k}\right.$ ) coordinates of these particles (for $k$ from 1 to $N$ ) are shown as the green dots in Fig. 5, and the normalized CSR forces $G_{k}=G_{H}\left(\tilde{x}_{k}, \tilde{z}_{k}, s\right)$ on these particles are plotted as the red dots in Fig. 5. These red dots are also depicted
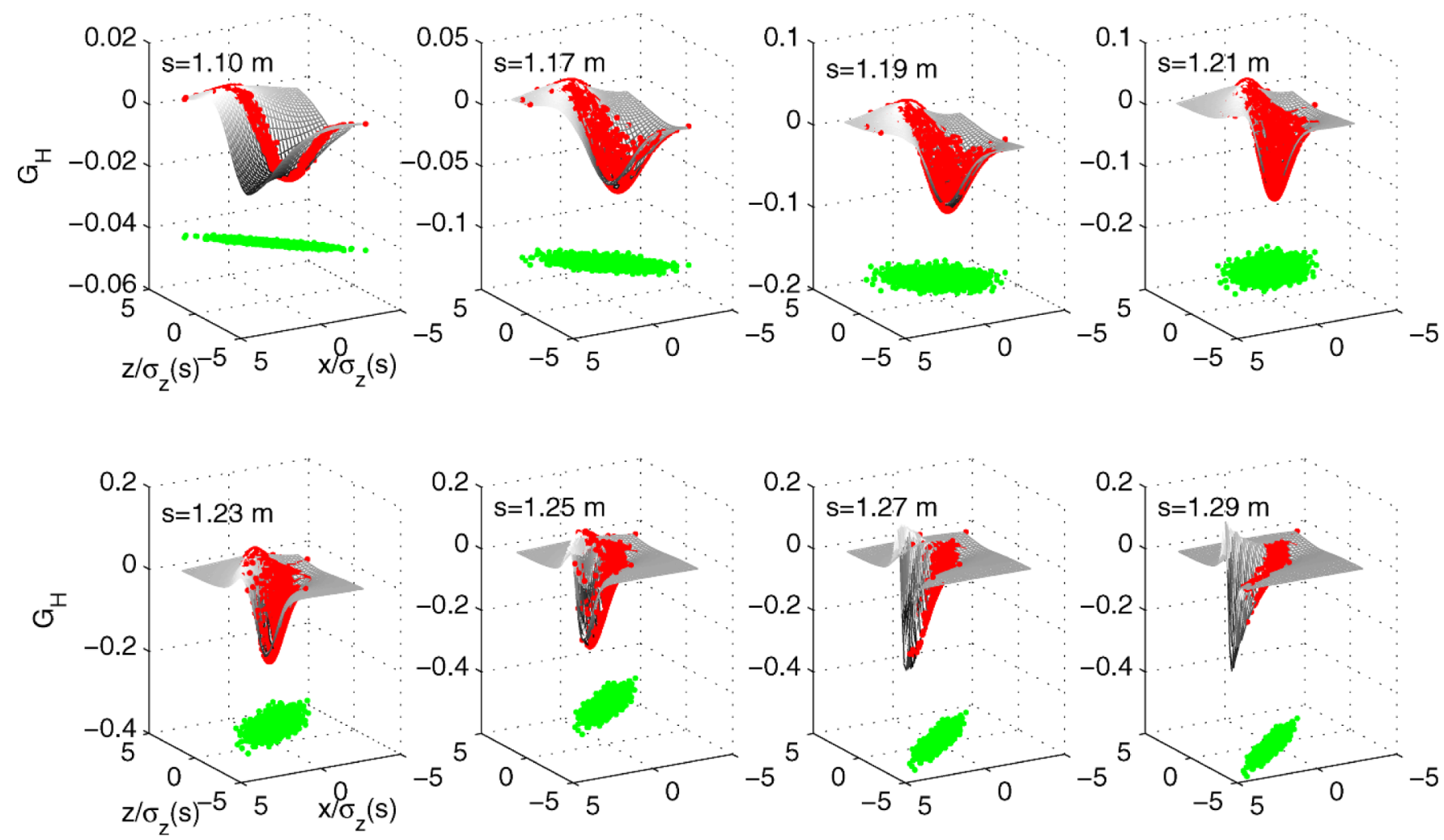

FIG. 5. (Color) $G_{H}(\tilde{x}, \tilde{z}, s)$ vs $(\tilde{x}, \tilde{z})$ at each $s$ around full compression for the thin-bunch case with $\epsilon_{n x 0}=1 \mathrm{~mm}-\mathrm{mrad}$ and $\sigma_{H 0}=$ $10^{-4}$. The gray mesh is $G_{H}(\tilde{x}, \tilde{z}, s)$ around the bunch as obtained from Eq. (134), and the green dots are $\left(\tilde{x}_{k}, \tilde{z}_{k}\right)$ coordinates of the 1000 representative particles of the bunch. The red dots are $G_{H}(\tilde{x}, \tilde{z}, s)$ on these representative particles. 

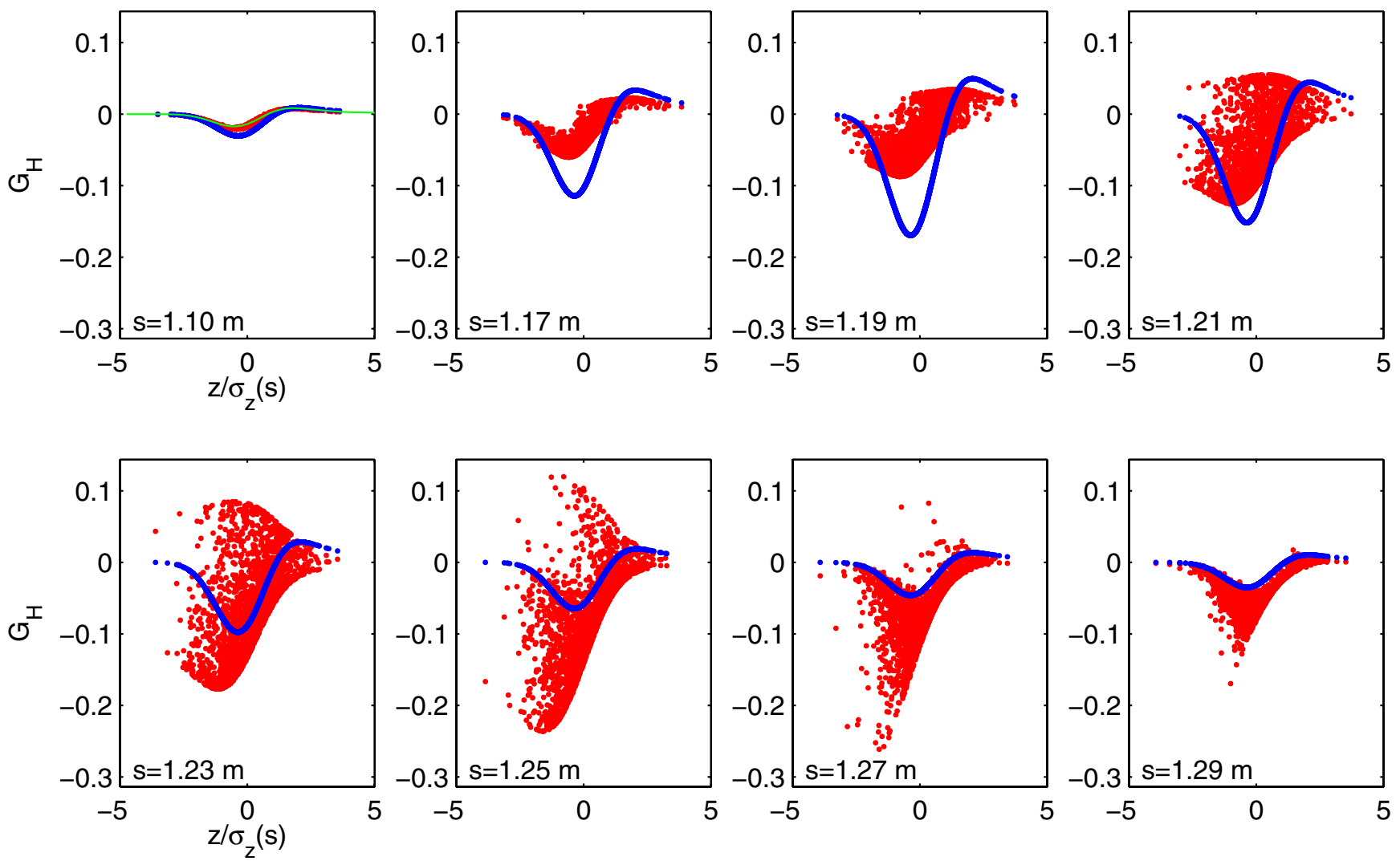

FIG. 6. (Color) $G_{H}(\tilde{x}, \tilde{z}, s)$ vs $\tilde{z}$ for the representative particle of the bunch at each $s$ for the thin-bunch case with $\epsilon_{n x 0}=1 \mathrm{~mm}-\mathrm{mrad}$ and $\sigma_{H 0}=10^{-4}$. The red dots are obtained from Eq. (134), and the blue line is the rigid-line bunch result in Eq. (140). The green line for $s=1.10 \mathrm{~m}$ case is the result from Eq. (128).

(a) mean of $\mathrm{G}_{\mathrm{H}}$ and $\mathrm{G}_{\mathrm{H} 0}$ vs. $\mathrm{s}$

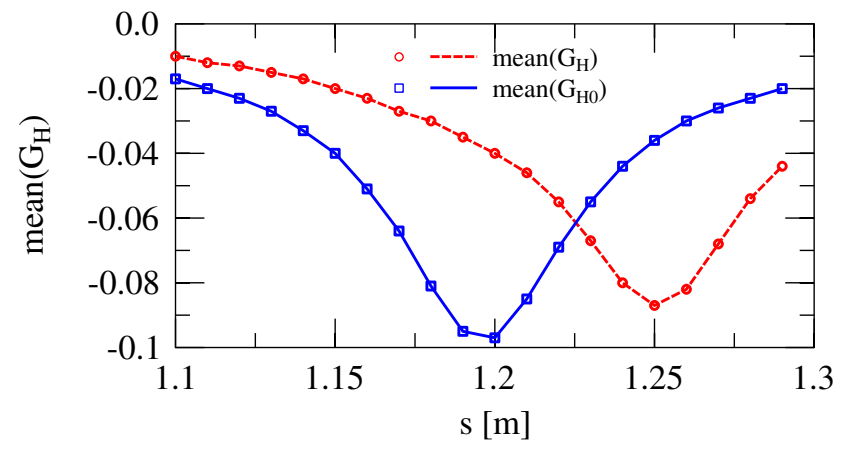

(b) maxmum and minimum of $\mathrm{G}_{\mathrm{H}}\left(\mathrm{x}_{\mathrm{k}}, \mathrm{z}_{\mathrm{k}}, \mathrm{s}\right)$ vs. $\mathrm{s}$

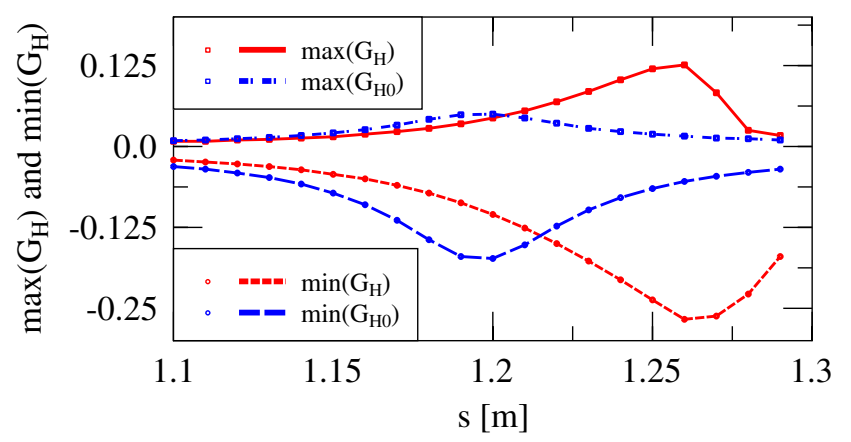

FIG. 7. (Color) Thin-bunch case: $\epsilon_{n \times 0}=1 \mathrm{~mm}$-mrad and $\sigma_{H 0}=$ $10^{-4}$. (a) Mean of $G_{H}\left(\tilde{x}_{k}, \tilde{z}_{k}, s\right)$ and $G_{H 0}\left(\tilde{z}_{k}, s\right)$ for the representative particles; and (b) maximum and minimum values of $G_{H}\left(\tilde{x}_{k}, \tilde{z}_{k}, s\right)$ and $G_{H 0}\left(\tilde{z}_{k}, s\right)$ for the representative particles vs path length $s$. 

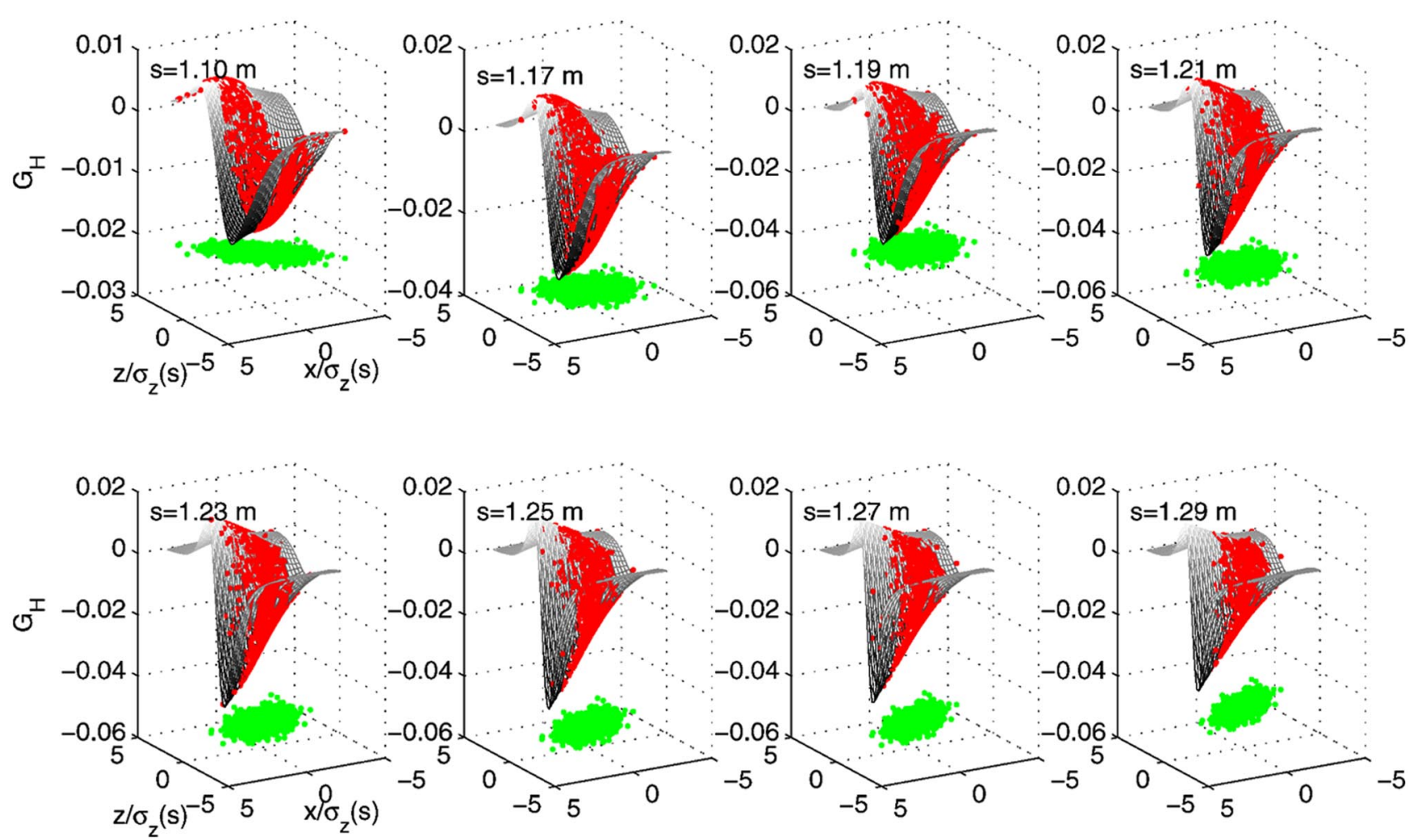

FIG. 8. (Color) $G_{H}(\tilde{x}, \tilde{z}, s)$ vs $(\tilde{x}, \tilde{z})$ at each $s$ around full compression for the thick-bunch case with $\epsilon_{n x 0}=10 \mathrm{~mm}$-mrad and $\sigma_{H 0}=$ $10^{-3}$. The gray mesh is $G_{H}(\tilde{x}, \tilde{z}, s)$ around the bunch as obtained from Eq. (134), and the green dots are $\left(\tilde{x}_{k}, \tilde{z}_{k}\right)$ coordinates of the 1000 representative particles of the bunch. The red dots are $G_{H}(\tilde{x}, \tilde{z}, s)$ on these representative particles.
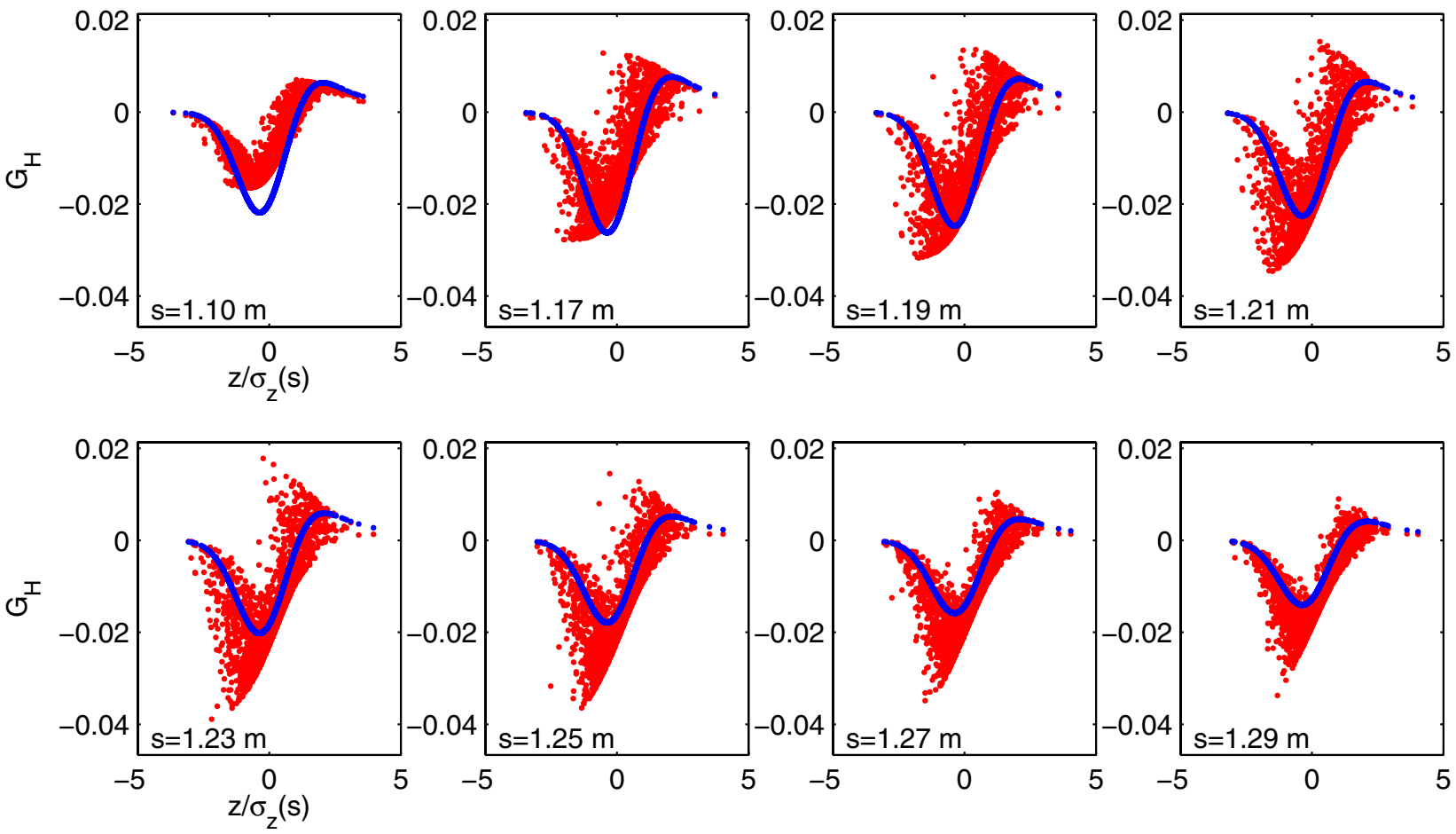

FIG. 9. (Color) $G_{H}(\tilde{x}, \tilde{z}, s)$ vs $\tilde{z}$ for the representative particle of the bunch at each $s$ for the thick-bunch case with $\epsilon_{n x 0}=1 \mathrm{~mm}$-mrad and $\sigma_{H 0}=10^{-3}$. The red dots are obtained from Eq. (134), and the blue line is the rigid-line bunch result in Eq. (140). 
in Fig. 6 as $G_{H}\left(\tilde{x}_{k}, \tilde{z}_{k}, s\right)$ vs $\tilde{z}_{k}$, which are compared with the rigid-line bunch result represented by the solid blue lines. The average of $G_{k}$ over these particles, $\langle G\rangle=$ $(1 / N) \sum_{k} G_{k}$, which is related to the canonical energy loss of the whole bunch due to the CSR interaction, is shown as the dashed red curve in Fig. 7(a) and is compared with the solid blue curve for the rigid-line bunch result. Similarly, the minimum and maximum values of $G_{k}$, indicating the spread of $G_{k}$ around $\langle G\rangle$, are plotted in Fig. 7(b). For the thick-bunch case, the behaviors of the longitudinal CSR force are shown in Figs. 8-10.

It is found that when the bunch is undercompressed $(\hat{\chi}>$ 0 ) with a moderate tilt, the major contribution to $\tilde{F}_{H}$ in Eq. (E30) comes from $I_{0}^{(-)}$as the result of the tail-head interaction represented by the retarded solution $z_{r 0}^{(-)}$. As the tilt gets stronger, the contribution from $I_{0}^{(+)}$to $\tilde{F}_{H}$ increases until its being comparable to that from $I_{0}^{(-)}$when the bunch is slightly over full compression. When the bunch is further overcompressed $(\hat{\chi}<0)$, the contribution from $I_{0}^{(-)}$subsides and $I_{0}^{(+)}$becomes dominant. This corresponds to the

(a) mean of $\mathrm{G}_{\mathrm{H}}$ and $\mathrm{G}_{\mathrm{H} 0}$ vs. $\mathrm{s}$

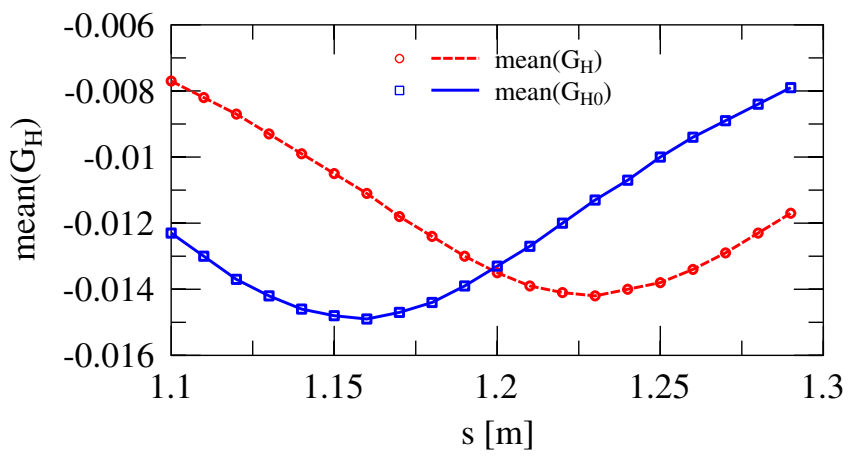

(b) maximum and minimum of $\mathrm{G}_{\mathrm{H}}\left(\mathrm{x}_{\mathrm{k}}, \mathrm{z}_{\mathrm{k}}, \mathrm{s}\right)$ vs. $\mathrm{s}$

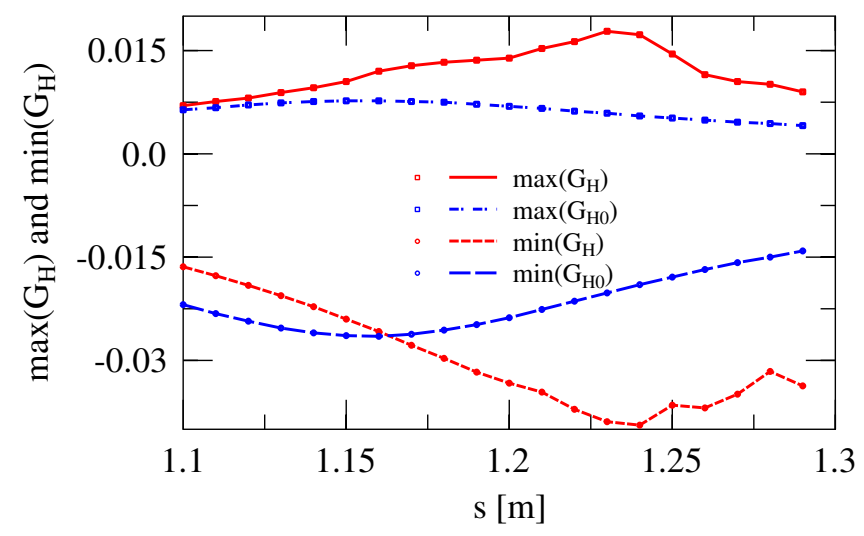

FIG. 10. (Color) Thick-bunch case: $\epsilon_{n x 0}=1 \mathrm{~mm}-\mathrm{mrad}$ and $\sigma_{H 0}=10^{-3}$. (a) Mean of $G_{H}\left(\tilde{x}_{k}, \tilde{z}_{k}, s\right)$ and $G_{H 0}\left(\tilde{z}_{k}, s\right)$ for the representative particles; and (b) maximum and minimum values of $G_{H}\left(\tilde{x}_{k}, \tilde{z}_{k}, s\right)$ and $G_{H 0}\left(\tilde{z}_{k}, s\right)$ for the representative particles vs path length $s$. contribution of retardation solution $z_{r 0}^{(+)}$, which again reflects the tail-head interaction, except that the bunch tail for an overcompressed bunch was originally at bunch head when $s=0$.

\section{Discussions}

In Figs. 5 and 8, we show how the longitudinal effective CSR force on a bunch varies as the bunch goes from undercompression to overcompression. When a thin bunch is mildly tilted at $s=1.10 \mathrm{~m}$, as shown in Fig. 6, the result of $\tilde{F}_{H}$ in Eq. (128) (green line) is a good approximation of the more rigorous result given in Eq. (E36) (red dots). As the compression gets stronger, the moderately tilted-thinbunch approximation used in Sec. VI A is no longer valid, and Figs. 5 and 6 show that $\tilde{F}_{H}$ on a test particle gets more sensitive to the particle's internal transverse position within the bunch. Meanwhile, both the average value and the spread of $\tilde{F}_{H}$ over the bunch increase and reach their peak shortly after the full compression point. As the bunch is further overcompressed, the strength of $\tilde{F}_{H}$ starts to reduce; so does the sensitivity of $\tilde{F}_{H}$ on the particles' transverse position.

Here is our present understanding of the behavior of the effective CSR forces in Figs. 5-10. First of all, Figs. 7(a) and 10(a) exhibit a time delay of the actual canonical energy loss by the whole bunch in its response to the bunch-length change, as compared to its counterpart case for the rigid-line bunch. This is a manifestation of the retardation effect. For example, the rigid-line bunch result (blue lines) in Fig. 7(a) shows that the maximum canonical energy loss occurs when the bunch length reaches its minimum at $s=1.2 \mathrm{~m}$. However, in reality, the bunch "sees" the strongest longitudinal collective radiation field only when it is at a certain path length downstream of $s=$ $1.2 \mathrm{~m}$. In our example, the maximum (canonical) energy loss occurs at $s \simeq 1.25 \mathrm{~m}$. This phenomenon can be understood from Fig. 11, which is the spacetime diagram of a bunch moving on a circular orbit in a $2 \mathrm{D}$ bending plane, where a test particle at $(x, z, s)$ receives EM fields generated from the interception of the bunch world volume (or worldline of source particles) with the past light cone of the test particle. It takes a finite range of path length for the information about the bunch-length-variation history to reach the bunch at the present time. This view also helps us to interpret the suppression of the magnitude of $\tilde{F}_{H}$ for a thin bunch under mild compression in Fig. 2 from the rigidline bunch result. The underlying cause is that the bunch at a given path length actually sees fields from itself generated at an upstream path length when the bunch length is longer. In Fig. 10(a), as a result of the role played by $\sigma_{z}^{\text {un }}(s)$ in Eq. (137), the minimum bunch length for a rigid-line bunch occurs at $s \simeq 1.15 \mathrm{~m}$ instead of $s=1.20 \mathrm{~m}$, and the maximum canonical energy loss happens at $s \simeq 1.23 \mathrm{~m}$. The data fluctuation in Fig. 10(b) for the maximum and 


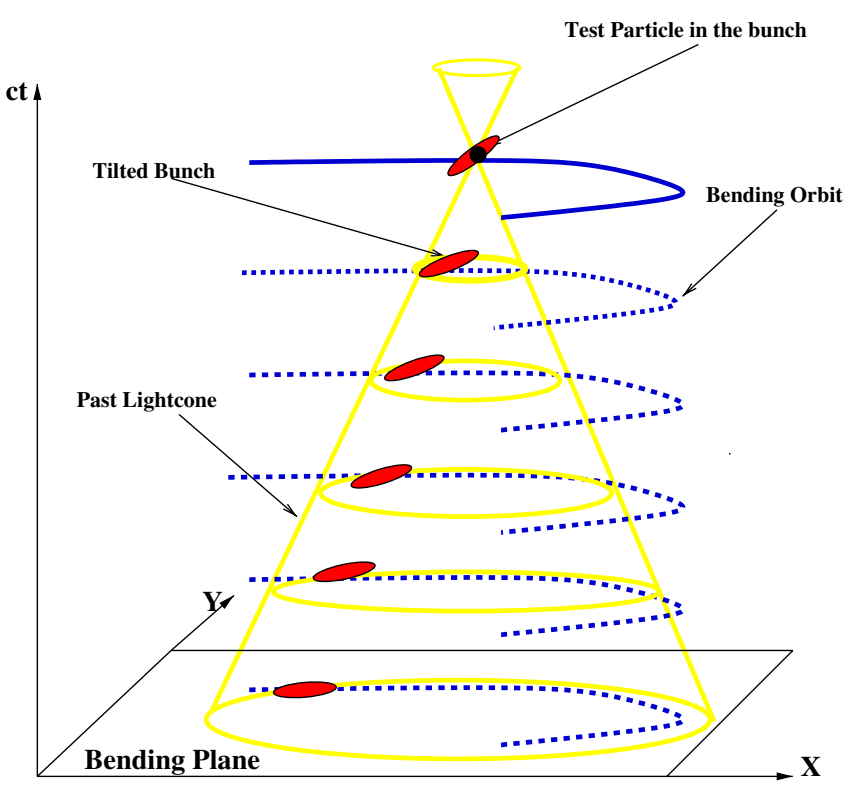

FIG. 11. (Color) Spacetime diagram of a test particle in a bunch interacting with fields generated from the interception of the worldline of the particles in the bunch and the past light cone of the test particle.

minimum value of $G_{H}$ is mainly caused by the random sampling of bunch phase space by a small number of particles.

Second, Figs. 6 and 9 show various levels of dependence of $\tilde{F}_{H}$ on the particles' transverse positions when the bunch is around full compression, especially around $s=1.25 \mathrm{~m}$. Mathematically, this is mainly originated from the dependence of retardation solution $z_{r 0}^{( \pm)}$in Eq. (69) on $x$ and $z$. More specifically, this can be seen from the $\left(x-\chi_{r} z\right)$ term in $\nu_{0}\left(x, z, s, s_{r}\right)$ of Eq. (E20), which is part of $\psi^{( \pm)}$in Eq. (E29). Another cause of the transverse sensitivity is the delicate selection of phase space $Z_{r 0}=\left(x_{r 0}, x_{r 0}^{\prime}, \delta_{H r 0}\right)$ according to Eq. (C27) for the CSR interaction, in which $\eta$ and $\eta_{\min }$ are given by Eqs. (C8) and (C16), respectively. At small $\Delta \bar{s}$ when $\chi_{r} \rightarrow 0$, one can see from Eq. (C16) that $\eta_{\min }$ can be small enough to select only a portion of $\left(x_{r 1}, z_{r 1}\right)$ in Eq. (C8). The sensitivity of such selection to $\chi x-z$ of the test particle can be seen from $\eta_{\min }\left(x, z, s, s_{r}\right)$ in Eq. (C16). A mild tilt is when $\chi_{r}$ is big enough such that $\eta_{\min } \gg \eta$. In this case, the whole $Z_{r 0}$ phase space at $s_{r}$ contributes to $\tilde{F}_{H}(x, z, s)$ and thus the sensitivity to $\left(x_{1}, z_{1}\right)$ is to large extent reduced.

The physical picture of the transverse sensitivity may also be partially understood from the single-particle radiation field. For a single particle undergoing relativistic circular motion with radius $R$, the distribution of its Lienard-Wiechert fields is highly concentrated in a belt $r>$ $R$ a little over 90 degrees from the direction of the bunch motion, which is called the radiation wave front, as can be seen from Fig. 4 of Ref. [20] and from Fig. 2 of Ref. [21]. Unlike the case of a relativistic source particle with uni- form motion when the Lorentz force on the test particle is $\gamma^{-2}$ times of that from a nonrelativistic source particle (transversely this is due to $\mathbf{E}$ and $\mathbf{B}$ cancellation), here the radial acceleration of the source particle causes the test particle to experience a centrifugal force. In addition, when the test particle crosses over the radiation wave front of the source particle, it will also experience a strong impulse of longitudinal force due to the longitudinal component of the electric field around the radiation wave front. As for the collective interaction of a bunch distribution, each test particle sees the superposition of such single-particle generated fields, where the strong fields from source particles on both sides of the test particle may be to a certain extent averaged out. When the bunch is thin and tilted a little over the fully compressed position, however, such an average can be rather imbalanced and the sensitivity to transverse position can be rather significant.

Note that in the canonical formulation of the CSR problem [12], the cancellation naturally takes place, and thus the transverse sensitivity of the centrifugal CSR force due to the singular $1 /\left|\mathbf{r}-\mathbf{r}^{\prime}\right|$ dependence of nearby-particle retarded potential is canceled by that of the transverse effect of the potential energy change which features the same transverse sensitivity. Despite this cancellation of the transverse sensitivities originated from the nearby-particle interaction, from this paper, we can see that around full compression, the residual effective longitudinal CSR force can also display strong transverse sensitivity. The origin of this sensitivity, though, is largely due to the delicate retardation when the bunch rotates to a specific geometric regime with respect to the direction of bunch motion, rather than due to the $1 /\left|\mathbf{r}-\mathbf{r}^{\prime}\right|$ integrand which exists regardless of the tilt of the bunch with respect to the reference orbit. In addition, we note that, contrary to the centrifugal spacecharge force, for which the front-to-back interaction $s_{r}>s$ contributes significantly [16], the energy-independent part of the longitudinal effective CSR force discussed in this paper is mainly contributed by the back-to-front $\left(s_{r} \leq s\right)$ interactions.

The geometrical feature of the CSR interaction is that a test particle sees the EM field generated from the source particles when the worldlines of the source particles intercept the past light cone of the test particle, and this interception depends on the curvature of the bunch orbit. In this study, by employing the bunch internal coordinates for the easy description of the linear optics, the above geometrical feature of the CSR interaction can only be reflected indirectly via the retardation solution, along with the delicate selection of the portion of $Z_{r 0}$ phase space for each $s_{r}$ in the evaluation of $\tilde{F}_{H}(x, z, s)$. The necessity of the proper description of the retarded phase space can cause potential challenge to the CSR simulations. For example, in our previous CSR simulation [22], even though the retardation was carefully treated to model the intersection of the past light cone with the bunch's world volume, the description 
of the bunch distribution using macroparticles was too crude to grasp the detailed phase space distribution at the interception around full compression. Recently, a computer code was developed [23] using the most natural way to account for the retardation effect. The code takes special care in the fine description of the bunch distribution, and calculates the CSR and space-charge forces by directly integrating over the curves where the bunch and the past light cone intersect. CSR effects in the unperturbed-source approximation have been extensively studied using this code [24,25] for realistic benchmark bunch compressors, where it was shown that the unperturbed-source approximation is a useful approximation for certain scenarios. The effect of transverse force has also been investigated in Ref. [24]. The benchmarking between our theory and simulations will be further pursued.

Notice that with the delayed response of the CSR force to the bunch length variation, the maximum CSR interaction occurs when the bunch is slightly overcompressed. In this situation, the particles with the highest energy-loss rate are those which were the head particles at $s=0$ and thus have negative initial energy offset for $u<0$. Such a picture would be altered if the delay of the response of the CSR force to bunch-size variation were not included.

Another remark is that, in the quantum mechanical treatment of synchrotron radiation for a single electron [26], the Coulomb gauge is used for the external potentials, and the change of the canonical energy (or Hamiltonian) by the electron is shown to be equal to the energy of real photons given off by the interaction process. Here in our classical treatment of CSR for a charged distribution, the Lorentz gauge is our natural choice of gauge. In this case, however, the explicit relation between the canonical energy loss of the bunch and the radiation energy given off by the bunch has not yet been established, and may need further study.

\section{Implication for chicane designs}

The designs of bunch compression chicanes for 4th generation light sources often demand minimal transverse-slice-emittance growth. One implication of the two-dimensional effect of the CSR forces, as studied in this paper, on these chicane designs is that the transverse sensitivity, or the sensitive dependence of the CSR forces on the particles' transverse position as displayed by the effective CSR forces around full compression, may have impacts on the growth of the transverse slice emittance and thus should be carefully examined in design calculations. Another implication is that the delayed response of the CSR force to bunch-size variation indicates that the undercompressed regime is more preferable for chicane designs, since in these cases the CSR forces are smaller in amplitude than those of a rigid-bunch model.

Besides situations around full compression, the mechanism that causes the transverse sensitivity of $\tilde{F}_{H}$, as pre- sented in this paper for a Gaussian bunch with an initial linear energy chirp, can also be present for an energymodulated bunch with mild tilt. As studied by recent research [27,28], a bunch entering a chicane can have some internal structure in the longitudinal phase space, often as a result of the initial longitudinal phase-space manipulation, nonlinear longitudinal optical transport, or the longitudinal space-charge effect. Such energy modulation may result in a local crossover, namely, the $x-z$ correlation for a local section in the bunch to cross over $\chi=0$ before the whole bunch reaches full compression. The effect of the sensitive retardation on the microbunching instability for a tilted bunch during compression requires further investigation.

From Eqs. (B13) and (B22), we can see that the transverse phase-space distribution is determined by both the longitudinal and transverse CSR forces. Our preliminary study shows that, for an energy-chirped bunch around full compression, the centripetal force term $\partial\left(\tilde{A}_{x}-\beta_{0} \tilde{\Phi}\right) / \partial x$ (see also [8]) possesses similar features of time delay (or retardation) and transverse sensitivity as those of the effective longitudinal force. Unlike the effective longitudinal CSR force generated at an arbitrary path length $s_{\tau}$, which impacts the change of $x^{\prime}$ at path length $s$ via $R_{26}\left(s_{\tau} \rightarrow s\right)$, the transverse effective CSR force generated at $s_{\tau}$ impacts $x^{\prime}$ at $s$ via $R_{22}\left(s_{\tau} \rightarrow s\right)$. Even though the transverse effective CSR force at $s_{\tau}$ is often $\left[\sigma_{z}\left(s_{\tau}\right) / R\right]^{1 / 3}$ times smaller in magnitude compared to that of the longitudinal CSR force, $R_{22}\left(s_{\tau} \rightarrow s\right)$ can be much larger than $R_{26}\left(s_{\tau} \rightarrow s\right)$, especially in the case when $s_{\tau}$ is inside the last bend of a chicane and emittance is measured at $s$ which is at the end of the chicane and when the bunch is critically compressed. Therefore the transverse effective force is an indispensable part in the understanding of the experimental observations of transverse phase space, and its impact on the transverse emittance growth needs to be carefully evaluated. For a complete picture of the bunch dynamics in a bending system, further investigations are also needed on the effects of the $\gamma_{0}^{-2}$ dependent terms, the effects of straight sections, and finally the effects of bunch vertical dimension on the CSR interaction.

\section{CONCLUSION}

In this paper, we studied the dynamics of an ultrarelativistic electron bunch moving on a curved orbit under collective interaction. The equations of motion were obtained from the Hamiltonian of an electron in the bunch, from which the Vlasov equation for the phase-space distribution of the bunch is derived. With the phase-space description chosen so as to explicitly apply the cancellation effect, the integral equation yielded from the Vlasov equation manifests clearly how the phase-space distribution is perturbed by the effective CSR forces. After the above formulas for the self-consistent dynamics were established, we focused on the analysis of the impact of bunch tilt on 
the effective longitudinal CSR force, which was carried out under the unperturbed-source approximation for a bunch with initial linear energy chirp. For a moderately tilted thin bunch with an initial Gaussian phase-space distribution, we presented the analytical expression of the effective longitudinal CSR force as a function of bunch tilt. These analytical results agree well with Dohlus' simulation results. For a thick bunch under bunch compression, our study yielded features such as retarded response of the canonical energy loss of the bunch to the bunch-length variation, and the strong dependence of the longitudinal effective CSR forces on the particles' transverse position around full compression. Many other aspects of the effective CSR forces for a tilted bunch, such as the behavior of the effective transverse CSR force, the behavior of the Coulomb terms, and the effective forces at the entrance and exit of a circular orbit, can be further studied along the approach developed in this paper. A full investigation of the CSR effect in a bunch-compression chicane requires a self-consistent numerical simulation, and we hope that the present study can help in benchmarking theory with simu- lation on the calculation of the CSR forces, and in understanding the behavior and interplay of the CSR forces.

\section{ACKNOWLEDGMENTS}

The author wishes to thank Robert Warnock for helpful discussions. This work is supported by the Department of Energy under Contract No. DE-AC05-84ER40150.

\section{APPENDIX A: TRANSPORT MATRICES}

For a bunch with low charge or long bunch length, we often can assume $F^{[f]}(X, s)=0$ throughout the beam line. Then Eq. (29) becomes

$$
\frac{d X}{d s}=M(s) X
$$

which describes the linear optical transport of particles. For $X(0)$ being the particle's phase-space variable at $s=0$, the solution of Eq. (A1) takes the form

$$
X(s)=R(s) X(0), \quad \text { with } R(s)=\left(\begin{array}{cccccc}
R_{11}(s) & R_{12}(s) & 0 & 0 & 0 & R_{16}(s) \\
R_{21}(s) & R_{22}(s) & 0 & 0 & 0 & R_{26}(s) \\
0 & 0 & R_{33}(s) & R_{34}(s) & 0 & 0 \\
0 & 0 & R_{43}(s) & R_{44}(s) & 0 & 0 \\
R_{51}(s) & R_{52}(s) & 0 & 0 & 1 & R_{56}(s) \\
0 & 0 & 0 & 0 & 0 & 1
\end{array}\right) \text {, }
$$

where $R(s)$ is the usual linear transport matrix for a beam line. For a bunch with initial Twiss parameters $\beta_{x 0}, \alpha_{x 0}, \beta_{y 0}, \alpha_{y 0}$ and an initial energy chirp $\delta_{E}=u z_{0}(u<0$ for a bunch under compression) imposed by an upstream rf cavity, one can further define the normalized initial phase-space variables $X_{0}=\left(x_{0}, x_{0}^{\prime}, y_{0}, y_{0}^{\prime}, z_{0}, \delta_{H 0}\right)^{T}$ :

$$
X_{0}(0)=A X(0), \quad \text { for } A=\left(\begin{array}{cccccc}
1 & 0 & 0 & 0 & 0 & 0 \\
\alpha_{x 0} / \beta_{x 0} & 1 & 0 & 0 & 0 & 0 \\
0 & 0 & 1 & 0 & 0 & 0 \\
0 & 0 & \alpha_{y 0} / \beta_{y 0} & 1 & 0 & 0 \\
0 & 0 & 0 & 0 & 1 & 0 \\
0 & 0 & 0 & 0 & -u & 1
\end{array}\right)
$$

Combining Eqs. (A2) and (A3), we have the transport of the phase-space vector from the initial normalized phase space to the phase space at $s$ :

$$
X(s)=\mathcal{R}(s) X_{0}(0) \quad \text { for } \mathcal{R}(s)=R(s) A^{-1}
$$

or

$$
\mathcal{R}(s)=\left(\begin{array}{cccccc}
R_{11}(s)-\frac{\alpha_{x 0}}{\beta_{x 0}} R_{12}(s) & R_{12}(s) & 0 & 0 & u R_{16}(s) & R_{16}(s) \\
R_{21}(s)-\frac{\alpha_{x 0}}{\beta_{x 0}} R_{22}(s) & R_{22}(s) & 0 & 0 & u R_{26}(s) & R_{26}(s) \\
0 & 0 & R_{33}(s)-\frac{\alpha_{y 0}}{\beta_{y 0}} R_{34}(s) & R_{34}(s) & 0 & 0 \\
0 & 0 & R_{43}(s)-\frac{\alpha_{y 0}}{\beta_{y 0}} R_{44}(s) & R_{44}(s) & 0 & 0 \\
R_{51}(s)-\frac{\alpha_{x 0}}{\beta_{x 0}} R_{52}(s) & R_{52}(s) & 0 & 0 & 1+u R_{56}(s) & R_{56}(s) \\
0 & 0 & 0 & 0 & u & 1
\end{array}\right) .
$$

Substituting Eq. (A4) into Eq. (A1), one finds that $\mathcal{R}(s)$ satisfies 


$$
\frac{d \mathcal{R}(s)}{d s}=M(s) \mathcal{R}(s) \quad \text { and } \quad \frac{d \mathcal{R}^{-1}(s)}{d s}=-\mathcal{R}^{-1}(s) M(s),
$$

where $d\left(\mathcal{R} \mathcal{R}^{-1}\right) / d s=0$ is used.

For nonzero effective CSR forces, let us define

$$
X_{0}(s)=\mathcal{R}^{-1}(s) X(s) \text {. }
$$

From Eqs. (A6) and (29), the change of $X_{0}$ is found to be driven by $F^{[f]}$ :

$$
\frac{d X_{0}}{d s}=\mathcal{R}^{-1}(s) F^{[f]}\left[\mathcal{R}(s) X_{0}, s\right] .
$$

Note that for vanishing effective CSR forces, $X_{0}(s)$ is a set of invariant phase-space variables, namely, $X_{0}(s)=X_{0}(0)$ when $F^{[f]}(X, s)=0$ for all $s$.

\section{APPENDIX B: VLASOV EQUATION}

In this section, the evolution equation for the charge distribution in our chosen phase space is set up based on the dynamical equations formulated in Sec. II. This evolution equation is then further transformed into an integral equation for an initially energy-chirped bunch. The relations of the phase-space distribution $f(X, s)$ and the physical quantities observed in the laboratory are also listed.

\section{Setting up the evolution equation}

The evolution of a charged-particle phase-space distribution in an external field is often studied using the Vlasov equation. The phase-space coordinates in the Vlasov equation are usually the position vector and kinetic momentum (or velocity) of a particle, which form a canonicalconjugate pair in the Hamiltonian. In the presence of (collisionless) collective interaction, the role of the kinetic momentum as the canonical conjugate of the position vector is replaced by that of the canonical momentum. Nonetheless, the form of the Lorentz force still allows the phase-space distribution to satisfy the MaxwellVlasov equation with $(\mathbf{x}, \mathbf{v})$ as a noncanonical pair. In this paper, since we choose the hybrid set $\left(x, x^{\prime}, y, y^{\prime}\right.$, $\left.z, \delta_{H}\right)$ as the phase-space variables, with $\left(x, x^{\prime}\right)$ and $\left(y, y^{\prime}\right)$ being noncanonical pairs whereas $\left(z, \delta_{H}\right)$ is a canonical one, we need to set up the phase-space evolution equation for the particular dynamical system described by Eq. (29).

From Eq. (29), we can see that the phase-space variables at $s$ and at $s+\Delta s$ are related to the first order of $\Delta s$ by

$$
X_{1} \equiv X(s+\Delta s)=X(s)+Y(X, s) \Delta s .
$$

Note that, similar to the derivation of the Maxwell-Vlasov equation, here the phase-space volume is conserved to the first order of $\Delta s$, namely,

$$
d X_{1}=\left[1+\Gamma^{[f]}(X, s) \Delta s\right] d X
$$

for

$$
\Gamma^{[f]}(X, s) \equiv \sum_{i=1}^{6} \frac{\partial Y_{i}(X, s)}{\partial X_{i}}
$$

Using Eq. (B2), and the conservation of the number of charged particles in an infinitesimal phase-space volume

$$
d N=f(X, s) d X=f\left(X_{1}, s+\Delta s\right) d X_{1},
$$

one gets the continuity equation for the dynamics given by Eq. (29):

$$
\frac{\partial f}{\partial s}+\sum_{i=1}^{6} Y_{i}(X, s) \frac{\partial f}{\partial X_{i}}+f \sum_{i=1}^{6} \frac{\partial Y_{i}}{\partial X_{i}}=0,
$$

in which $\sum_{i} \partial Y_{i} / \partial X_{i}=\partial F_{z} / \partial z$. With the effect of $F_{z}$ negligible, we obtain the Vlasov equation from Eq. (29),

$$
\frac{\partial f}{\partial s}+\sum_{i=1}^{6} Y_{i}(X, s) \frac{\partial f}{\partial X_{i}}=0 .
$$

\section{Integral equation for an energy-chirped bunch}

Following the analysis in Refs. [2,3], we now construct the integral equation using our phase-space evolution equation in Eq. (B6). This integral equation shows how the particle distribution in the normalized phase space, which is invariant under design linear optics, is perturbed by the effective CSR forces.

For a bunch with an initial energy chirp $\delta_{E}=u z(u<0$ for a bunch under compression) imposed by an upstream rf cavity, let $\rho\left(X_{0}, s\right)$ be the particle phase-space distribution for the normalized phase-space variables $X_{0}$, as introduced in Appendix A. From Eqs. (A7) we have

$$
f(X, s)=\rho\left[X_{0}=\mathcal{R}^{-1}(s) X, s\right],
$$

and from Eq. (A6) we have

$$
\frac{\partial f(X, s)}{\partial s}=\frac{\partial \rho\left(X_{0}, s\right)}{\partial s}-\left[\mathcal{R}^{-1}(s) M(s) X\right]_{i} \frac{\partial \rho\left(X_{0}, s\right)}{\partial\left(X_{0}\right)_{i}}
$$

and

$$
\frac{\partial f(X, s)}{\partial X_{i}}=\left[\mathcal{R}^{-1}(s)\right]_{j i} \frac{\partial \rho\left(X_{0}, s\right)}{\partial\left(X_{0}\right)_{j}} .
$$

Therefore the Vlasov equation for $\rho\left(X_{0}, s\right)$ can be obtained from Eq. (B6):

$$
\frac{\partial \rho\left(X_{0}, s\right)}{\partial s}+\left[\mathcal{R}^{-1}(s) F^{[f]}\left(\mathcal{R}(s) X_{0}, s\right)\right]_{i} \frac{\partial \rho\left(X_{0}, s\right)}{\partial\left(X_{0}\right)_{i}}=0,
$$

which is equivalent to the integral equation 


$$
\begin{aligned}
\rho\left(X_{0}, s\right)= & \rho\left(X_{0}, 0\right)-\int_{0}^{s} d s_{\tau}\left\{\mathcal{R}^{-1}\left(s_{\tau}\right) F^{[f]}\left[\mathcal{R}\left(s_{\tau}\right) X_{0}, s_{\tau}\right]\right\}_{i} \\
& \times \frac{\partial \rho\left(X_{0}, s_{\tau}\right)}{\partial\left(X_{0}\right)_{i}} .
\end{aligned}
$$

Denoting $X_{\tau}=\mathcal{R}\left(s_{\tau}\right) X_{0}$, and using

$$
\frac{\partial \rho\left(X_{0}, s_{\tau}\right)}{\partial\left(X_{0}\right)_{i}}=\left[\mathcal{R}\left(s_{\tau}\right)\right]_{j i} \frac{\partial f\left(X_{\tau}, s_{\tau}\right)}{\partial\left(X_{\tau}\right)_{j}},
$$

we can convert Eq. (B11) into an integral equation for $f(X, s)$ :

$$
f(X, s)=f^{(0)}(X, s)-\int_{0}^{s} d s_{\tau}\left[F^{[f]}\left(X_{\tau}, s_{\tau}\right)\right]_{i} \frac{\partial f\left(X_{\tau}, s_{\tau}\right)}{\partial\left(X_{\tau}\right)_{i}},
$$

for $\quad X_{\tau}=\left[\mathcal{R}\left(s_{\tau} \rightarrow s\right)\right]^{-1} X \quad$ with $\quad \mathcal{R}\left(s_{\tau} \rightarrow s\right)=$ $\mathcal{R}(s) \mathcal{R}^{-1}\left(s_{\tau}\right)$. Here

$$
f^{(0)}(X, s)=\rho\left[\mathcal{R}^{-1}(s) X, 0\right]
$$

gives the phase-space distribution under nominal optical transport, and the integral in Eq. (B13) gives the impact of the effective forces during $0 \leq s_{\tau} \leq s$ on $f(X, s)$.

\section{Small perturbation on a stable distribution}

Here we look at the evolution of a small initial perturbation on a stable phase-space distribution $f_{0}(X, s)$ :

$$
f(X, s)=f_{0}(X, s)+f_{1}(X, s),
$$

where $f_{0}(X, s)$ is such that $F^{\left[f_{0}\right]}(X, s)$ is negligible for $s$ along the beam line of interest and thus $f_{0}\left(X_{\tau}, s_{\tau}\right) \simeq$ $f_{0}\left[\mathcal{R}^{-1}\left(s_{\tau}\right) X_{\tau}, 0\right]$. A linearized integral equation can be reduced from Eq. (B13):

$$
f_{1}(X, s)=f_{1}^{(0)}(X, s)-\int_{0}^{s} d s_{\tau}\left[F^{\left[f_{1}\right]}\left(X_{\tau}, s_{\tau}\right)\right]_{j} \frac{\partial f_{0}\left(X_{\tau}, s_{\tau}\right)}{\partial\left(X_{\tau}\right)_{j}}
$$

with $f_{1}^{(0)}(X, s)=f_{1}\left[\mathcal{R}^{-1}(s) X, 0\right]$. The Fourier component of the perturbed longitudinal distribution at $s$ is [2,3]

$g_{1}(k, s)=\frac{1}{2 \pi} \int d X e^{-i k z} f_{1}(X, s)=g_{1}^{(0)}(k, s)+\Delta g_{1}(k, s)$,

where for $\rho_{0,1}\left(X_{0}, 0\right)=f_{0,1}\left[\mathcal{R}(s) X_{0}, 0\right]$, and using $d X=$ $d X_{\tau}=d X_{0}$, one has

$$
g_{1}^{(0)}(k, s)=\frac{1}{2 \pi} \int d X_{0} e^{-i k z\left(X_{0}, s\right)} \rho_{1}\left(X_{0}, 0\right)
$$

and

$$
\begin{aligned}
\Delta g_{1}(k, s)= & \frac{1}{2 \pi} \int_{0}^{s} d s_{\tau} \int d X_{0} e^{-i k z\left(X_{0}, s\right)} \rho_{0}\left(X_{0}, 0\right)(-i k) R_{5 j} \\
& \times\left(s_{\tau} \rightarrow s\right) F_{j}^{\left[f_{1}\right]}\left[\mathcal{R}\left(s_{\tau}\right) X_{0}, s_{\tau}\right],
\end{aligned}
$$

with $z\left(X_{0}, s\right)=\mathcal{R}_{5 i}(s)\left(X_{0}\right)_{i}$. When only the $j=6$ term is dominant and other terms are negligible, Eq. (B19) reduces to the perturbative version of Eq. 14 in Ref. [3]. Note that if $F_{z}$ in Eq. (26) were included in Eq. (B19), its contribution would be negligible when

$$
\left(s-s_{\tau}\right) / \gamma_{0}^{2} \ll R_{56}\left(s_{\tau} \rightarrow s\right)
$$

for $0 \leq s_{\tau} \leq s$.

\section{Relation to measurable distributions}

Because of the unconventional use of $\delta_{H}$ as a dynamical variable in Eqs. (29) and (B13), it is necessary for us to show how the phase-space distribution $f(X, s)$ is related to the distributions measured in the laboratory.

First, the energy spectrum is often measured at a high dispersion point $s=s_{D}$, where one measures the horizontal charge-density distribution, which is related to $f(X, s)$ by

$$
\lambda_{x}\left(x, s_{D}\right)=\int f\left(X, s_{D}\right) d x^{\prime} d y d y^{\prime} d z d \delta_{H}
$$

Note that, when the effective CSR forces are negligible, $\lambda_{x}\left(x, s_{D}\right)$ mainly measures the spread of $\delta_{H}$ at $s_{D}$.

Second, the horizontal emittance is often measured in a nondispersive region at $s=s_{\epsilon}$, which gives the rms area for the following horizontal phase-space distribution:

$$
\rho_{x}\left(x, x^{\prime}, s_{\epsilon}\right)=\int f\left(X, s_{\epsilon}\right) d y d y^{\prime} d z d \delta_{H}
$$

Since according to Eq. (B13), $f\left(X, s_{\epsilon}\right)$ in Eq. (B22) is fully determined by the effective CSR forces and the initial distribution $f(X, 0)$, the horizontal emittance is only perturbed by the effective CSR forces.

Next, the longitudinal density distribution is yielded from $f(X, s)$ by

$$
\lambda_{z}(z, s)=\int f(X, s) d x d x^{\prime} d y d y^{\prime} d \delta_{H}
$$

Similar to the horizontal emittance, here $\lambda_{z}(z, s)$ is also perturbed by the effective CSR forces.

However, unlike $\lambda_{x}\left(x, s_{D}\right), \rho_{x}\left(x, x^{\prime}, s_{\epsilon}\right)$, and $\lambda_{z}(z, s)$ which can be determined by $f(X, s)$ with the canonical energy offset $\delta_{H}$ as a dynamical variable, the actual longitudinal phase-space distribution $\rho_{z}\left(z, \delta_{E}, s\right)$, with $\delta_{E}$ being the kinetic energy offset, is related to the scalar potential on the test particles at $s$ following Eq. (41): 


$$
\begin{aligned}
\rho_{z}\left(z, \delta_{E}, s\right)= & \int f\left(x, x^{\prime}, y, y^{\prime}, z, \delta_{E}+\tilde{\Phi}(x, y, z, s)\right. \\
& \left.-\frac{1}{2 \gamma_{0}^{2}}, s\right) d x d x^{\prime} d y d y^{\prime} .
\end{aligned}
$$

Since $\tilde{\Phi}(x, y, z, s)$ is a functional of $f(X, s)$, the dependence of $\rho_{z}\left(z, \delta_{E}, s\right)$ on $f(X, s)$ cannot be expressed explicitly.

\section{APPENDIX C: SOME DETAILS ON THE RETARDATION SOLUTION}

\section{The coefficients of the quadratic equation $P(\Delta \bar{z})=0$}

In Sec. IV, the expression $P\left(x, z, s ; X_{r 0}, s_{r}\right)$ in Eq. (50) is reduced to a quadratic function of $\Delta \bar{z}$ in Eq. (61). One can show that the coefficients in Eq. (61) are

$$
\begin{aligned}
& a=1-\left(\beta_{0} \xi_{r}\right)^{2} \\
& b=\Delta \bar{s}+\bar{z}_{r 1}+\beta_{0}^{2} \hat{\xi}_{r}\left[\left(\hat{x}-\hat{x}_{r 1}-\hat{\xi}_{r} \bar{z}\right)-2(1+\hat{x}) \sin ^{2} \frac{\Delta \bar{s}}{2}\right] \\
& c \simeq\left(\frac{\Delta \bar{s}}{\gamma_{0}}\right)^{2}+\frac{(\Delta \bar{s})^{4}}{12}+2 \Delta \bar{s} \bar{z}_{r 1}+\left(\bar{z}_{r 1}\right)^{2}-\beta_{0}^{2}\left(\hat{x}-\hat{x}_{r 1}-\hat{\xi}_{r} \bar{z}\right)^{2}-\left[\hat{x}+(1+\hat{x})\left(\hat{x}_{r 1}+\hat{\xi}_{r} \bar{z}\right)\right]\left(2 \beta_{0} \sin \frac{\Delta \bar{s}}{2}\right)^{2},
\end{aligned}
$$

with

$$
\left(\bar{z}_{1}, \bar{z}_{r 1}\right)=\frac{1}{\left|R_{0}\right|}\left(z_{1}, z_{r 1}\right), \quad \text { and } \quad\left(\hat{x}_{1}, \hat{x}_{r 1}\right)=\frac{1}{R_{0}}\left(x_{1}, x_{r 1}\right)
$$

for $\left(z_{1}, z_{r 1}\right)$ and $\left(x_{1}, x_{r 1}\right)$ in Eqs. (53) and (56). Under the approximations

$$
\begin{gathered}
\sin (\Delta \bar{s} / 2) \simeq \Delta \bar{s} / 2, \quad \gamma_{0} \Delta \bar{s} \ll 1, \\
\beta_{0}^{2} \simeq 1, \quad 1+\hat{x} \simeq 1,
\end{gathered}
$$

the terms in Eq. $(\mathrm{C} 1)$ can be grouped as

$$
b \simeq b_{0}+b_{1}, \quad c \simeq c_{0}+c_{1},
$$

where $b_{0}$ and $c_{0}$ are values of $b$ and $c$ when $\hat{x}_{r 1}=\bar{z}_{r 1}=0$ :

$$
\begin{aligned}
& b_{0}=\Delta \bar{s}\left(1-\frac{\hat{\xi}_{r} \Delta \bar{s}}{2}\right)+\hat{\xi}_{r}\left(\hat{x}-\hat{\xi}_{r} \bar{z}\right), \\
& c_{0}=\frac{(\Delta \bar{s})^{4}}{12}-\left(\hat{x}-\hat{\xi}_{r} \bar{z}\right)^{2}-\left(\hat{x}+\hat{\xi}_{r} \bar{z}\right)(\Delta \bar{s})^{2},
\end{aligned}
$$

and $b_{1}$ and $c_{1}$ are related to the source particle's initial intrinsic offset via $z_{r 1}$ and $x_{r 1}$ in Eq. (53):

$$
\left\{\begin{array}{l}
b_{1}=\bar{z}_{r 1}-\hat{\xi}_{r} \hat{x}_{r 1}, \\
c_{1}=2 \Delta \bar{s} \bar{z}_{r 1}+\left(\bar{z}_{r 1}\right)^{2}+2 \hat{x}_{r 1}\left(\hat{x}-\hat{\xi}_{r} \bar{z}\right)-\left(\hat{x}_{r 1}\right)^{2}-\hat{x}_{r 1}(\Delta \bar{s})^{2} .
\end{array}\right.
$$

As shown in Eqs. (62)-(64), the roots of $P=0$ involve

$$
\begin{aligned}
& \omega=b^{2}-a c=\omega_{0}+\omega_{1} \\
& \text { with }\left\{\begin{array}{l}
\omega_{0}=\left(b_{0}\right)^{2}-a c_{0}, \\
\omega_{1}=2 b_{0} b_{1}+\left(b_{1}\right)^{2}-a c_{1} .
\end{array}\right.
\end{aligned}
$$

Note that $\omega_{1}$ consists of all terms depending on the intrinsic parameters $x_{r 1}$ and $z_{r 1}$.

\section{Existence of solutions: $\theta\left(b^{2}-a c\right)=1$}

The solutions to $P=0$, which are $\Delta \bar{z}^{( \pm)}$in Eq. (63) or $\bar{z}_{r 0}^{( \pm)}$in Eqs. (66) or (68), exist only when $\omega=b^{2}-a c \geq$ 0 , or $\theta\left(b^{2}-a c\right)=1$. When $(x, z, s)$ for a test particle are given, and when one of the integral variables in Eq. (74) the path length $s_{r}$ for the source particle-is given, this condition defines the range of $Z_{r 0}$ in the integrals in Eq. (74).

Using Eq. (56), let us define $\eta=\eta\left(x_{r 0}, x_{r 0}^{\prime}, \delta_{H r 0}\right)$ as

$$
\eta=\hat{\chi}_{r} \hat{x}_{r 1}-\bar{z}_{r 1}=\sum_{j=1,2,6} \alpha_{j}\left(s_{r}\right)\left(Z_{r 0}\right)_{j}
$$

for $\left(Z_{r 0}\right)_{j=1,2,6}=\left(x_{r 0}, x_{r 0}^{\prime}, \delta_{H r 0}\right)$ and

$$
\alpha_{j}\left(s_{r}\right)=\frac{\chi_{r} \mathcal{R}_{1 j}\left(s_{r}\right)-\mathcal{R}_{5 j}\left(s_{r}\right)}{\left|R_{0}\right|} .
$$

Then $\theta\left(b^{2}-a c\right)=1$ requires Eq. (C7) to satisfy

$$
\begin{aligned}
\left(\omega_{0}+\omega_{1}\right) \hat{\chi}_{r}^{2} / \beta_{0}^{2} & =\eta^{2}-2 A \eta+B \\
& =(\eta-A)^{2}-\left(A^{2}-B\right)>0
\end{aligned}
$$

with

$$
A \simeq \Delta \bar{s}\left(1-\hat{\chi}_{r} \Delta \bar{s} / 2\right)+\left(\hat{\chi}_{r} \hat{x}-\bar{z}\right), \quad B=\chi_{r}^{2} \omega_{0} / \beta_{0}^{2},
$$

and

$$
A^{2}-B \simeq\left(\beta_{0}^{2}-\chi_{r}^{2}\right) \Delta \bar{s}^{2}\left(1-\frac{\Delta \bar{s}^{2}}{3}+2 \hat{x}\right) .
$$

For a nontilted bunch, $\hat{\chi}_{r}^{2}>\beta_{0}^{2}$, we have $A^{2}-B<0$ in Eq. (C10). Consequently, $\theta\left(b^{2}-a c\right)=1$ for arbitrary $\eta$. Conversely, for a tilted bunch with $\hat{\chi}_{r}^{2}<\beta_{0}^{2}$, one has $A^{2}-$ $B>0$ in Eq. (C10), and hence can write

$$
\left(\omega_{0}+\omega_{1}\right) \hat{\chi}_{r}^{2}=\beta_{0}^{2}\left(\eta_{\min }-\eta\right)\left(\eta_{\max }-\eta\right)
$$

for 


$$
\eta_{\min }=A-\sqrt{A^{2}-B} \quad \text { and } \quad \eta_{\max }=A+\sqrt{A^{2}-B} .
$$

(C14)

Thus, Eq. (C13) yielding positive value imposes a finite range for $\eta$ :

$$
\eta \leq \eta_{\min } \quad \text { or } \quad \eta \geq \eta_{\max }
$$

with the end limits $\eta_{\min }$ and $\eta_{\max }$ being functions of $\left(x, z, s, s_{r}\right)$. For $\Delta \bar{s}>0$, these end limits are approximately

$$
\begin{aligned}
& \eta_{\min } \simeq\left(\frac{\Delta \bar{s} \hat{\chi}_{r}}{2}+\hat{x}\right)\left(\hat{\chi}_{r}-\Delta \bar{s}\right)+\frac{(\Delta \bar{s})^{3}}{6}-\bar{z}+\frac{\Delta \bar{s}}{2 \gamma_{0}^{2}}, \\
& \eta_{\max } \simeq 2 \Delta \bar{s},
\end{aligned}
$$

in which $\hat{\chi}_{r}-\Delta \bar{s} \simeq \hat{\chi}$ according to Eq. (D3), and for $\Delta \bar{s}<$ 0

$$
\begin{aligned}
& \eta_{\min } \simeq-2|\Delta \bar{s}| \\
& \eta_{\max } \simeq\left(-\frac{|\Delta \bar{s}| \hat{\chi}_{r}}{2}+\hat{x}\right)\left(\hat{\chi}_{r}+|\Delta \bar{s}|\right)-\frac{|\Delta \bar{s}|^{3}}{6}-\bar{z}-\frac{|\Delta \bar{s}|}{2 \gamma_{0}^{2}} .
\end{aligned}
$$

\section{Selection of retardation solutions: $\theta\left(Q^{( \pm)}\right)=1$}

For a source particle in a titled bunch to emit EM fields from the path length $s_{r}$, which interacts with the test particle at $(x, z, s)$, we have identified in Eqs. (66) and (68) the longitudinal position, $\bar{z}_{r 0}^{( \pm)}$, of the source particle in terms of $\left(x_{r 0}, x_{r 0}^{\prime}, \delta_{H r 0}\right)$ in its initial phase space, with $\bar{z}_{r 0}^{( \pm)}$either the "advanced" or "retarded" solution. In Sec. C 2, the range of $\left(x_{r 0}, x_{r 0}^{\prime}, \delta_{H r 0}\right)$ for the existence of $\bar{z}_{r 0}^{( \pm)}$is solved in terms of the range of $\eta$ in Eq. (C8). In the following, we will further refine the limit on the initial phase space so as to exclude the advanced solution and keep only the retarded one.

For $\Delta z^{( \pm)}$in Eq. (63), the retardation solution requires

$$
Q^{( \pm)}=\Delta s+z_{r 1}-\Delta z^{( \pm)}>0,
$$

which can be further written as

$$
\bar{Q}^{( \pm)}=\frac{Q^{( \pm)}}{\left|R_{0}\right|}=q_{0}\left[C_{0}(A-\eta) \pm \sqrt{\eta^{2}-2 A \eta+B}\right]>0
$$

with $A$ and $B$ given in Eq. (C11), and

$$
C_{0}=\frac{\beta_{0}}{\left|\hat{\chi}_{r}\right|}, \quad q_{0}=\frac{\left|\hat{\chi}_{r}\right|}{\beta_{0}^{2}-\hat{\chi}_{r}^{2}} .
$$

We will find that the quadratic form

$$
E(\eta)=\left(C_{0}^{2}-1\right) \eta^{2}-2 A\left(C_{0}^{2}-1\right) \eta+\left(A^{2} C_{0}^{2}-B\right)
$$

and its determinant

$$
D_{0}=\left(1-C_{0}^{2}\right)\left(A^{2}-B\right)
$$

are useful for studying Eq. (C19).

a. Nontilted bunch: $\chi_{r}^{2}>\beta_{0}^{2}$

In this case, one has $0<C_{0}<1, q_{0}<0$, and from Eq. (C12) one also has $A^{2}-B<0$. Thus $\eta^{2}-2 A \eta+$ $B>0$ is automatically satisfied (see Sec. C 2). From Eq. (C19), $Q^{(+)}>0$ holds only when

$$
\sqrt{\eta^{2}-2 A \eta+B}<C_{0}(\eta-A)
$$

which has no solution for $\eta<A$. For $\eta>A$, Eq. (C23) requires $E(\eta)>0$, which again has no solution due to $C_{0}^{2}<1$ and $D_{0}<0$. On the other hand, one can show that $Q^{(-)}>0$ holds for $\eta<A$. For $\eta>A, Q^{(-)}>0$ requires

$$
\sqrt{\eta^{2}-2 A \eta+B}>C_{0}(A-\eta),
$$

or $E(\eta)<0$, which is satisfied since $D_{0}<0$ and $C_{0}^{2}-1<$ 0 . As a result, for $\chi_{r}^{2}>\beta_{0}^{2}, z_{r 0}^{(-)}$is always a retarded solution for either $\Delta s \geq 0$ or $\Delta s<0$, while $z_{r 0}^{(+)}$is always excluded.

\section{b. Tilted bunch: $\chi_{r}^{2}<\beta_{0}^{2}$}

We now have $C_{0}>1, q_{0}>0$, and $A^{2}-B>0$. In this case, $\eta^{2}-2 A \eta+B>0$ holds only when $\eta<\eta_{\min }$, or $\eta>\eta_{\max }$ [see Eq. (C15)]. From Eq. (C19), we have $Q^{(+)}>0$ only when

$$
\sqrt{\eta^{2}-2 A \eta+B}>C_{0}(\eta-A)
$$

which is automatically satisfied for $\eta<\eta_{\min }$. For $\eta>$ $\eta_{\max }$, Eq. (C25) requires $E(\eta)<0$, which has no solution due to $\left(C_{0}^{2}-1\right)>0$ and $D_{0}<0$. Similarly, $Q^{(-)}>0$ holds when

$$
\sqrt{\eta^{2}-2 A \eta+B}<C_{0}(A-\eta)
$$

For $\eta>\eta_{\max }$, Eq. (C26) has no solution since $A-\eta<0$. However, for $\eta<\eta_{\min }$, Eq. (C26) requires $E(\eta)>0$, which is satisfied due to $\left(C_{0}^{2}-1\right)>0$ and $D_{0}<0$. Consequently, for either $\Delta \bar{s}<0$ or $\Delta \bar{s}>0$, both $z_{r 0}^{(+)}$and $z_{r 0}^{(-)}$are retarded solutions when

$$
\eta=\eta\left(x_{r 0}, x_{r 0}^{\prime}, \delta_{H r 0}\right) \leq \eta_{\min }\left(x, z, s, s_{r}\right) .
$$

The range of $\eta>\eta_{\max }$ in Eq. (C15) is excluded due to the retardation requirement.

\section{APPENDIX D: MORE ON THE RETARDED TILTING FACTOR}

From the definition of $\chi\left(s_{r}\right)$ and $R_{56}^{\prime}(s)=$ $-R_{16}(s) / R_{0}(s)$, one gets 


$$
\begin{aligned}
\chi\left(s_{r}\right)= & \frac{1+u R_{56}(s-\Delta s)}{u R_{16}(s-\Delta s)} \simeq \frac{R_{16}(s)}{R_{16}(s-\Delta s)} \\
& \times\left[\chi(s)+\frac{\Delta s}{R_{0}(s)}-\frac{R_{26}(s)}{2 R_{16}(s) R_{0}(s)}(\Delta s)^{2}\right],
\end{aligned}
$$

where

$$
\frac{R_{16}(s-\Delta s)}{R_{16}(s)} \simeq 1-\left(\frac{R_{26}(s)\left|R_{0}(s)\right|}{R_{16}(s)}\right) \Delta \bar{s}+\frac{R_{0}(s)}{2 R_{16}(s)}(\Delta \bar{s})^{2} .
$$

Equation (D1) further yields

$$
\hat{\chi}\left(s_{r}\right)-\frac{\Delta \bar{s}}{2} \simeq \frac{R_{16}(s)}{R_{16}\left(s_{r}\right)}\left(\hat{\chi}(s)+\frac{\Delta \bar{s}}{2}-\frac{R_{0}(s)}{4 R_{16}(s)}(\Delta \bar{s})^{3}\right) .
$$

For $O\left[R_{0} \Delta \bar{s}^{2} / R_{16}(s)\right] \ll 1$, Eq. (D3) becomes

$$
\begin{aligned}
\hat{R}_{16}\left(s_{r}\right)\left(\hat{\chi}_{r}-\frac{\Delta \bar{s}}{2}\right) & =\mathcal{R}_{55}\left(s_{r}\right)\left(1-\frac{\Delta \bar{s}}{2 \hat{\chi}_{r}}\right) \\
& \simeq \mathcal{R}_{55}(s)\left(1+\frac{\Delta \bar{s}}{2 \hat{\chi}(s)}\right) .
\end{aligned}
$$

It can also be shown that

$$
\begin{aligned}
& \left|u R_{16}\left(s_{r}\right)\right| \sqrt{\left(\hat{\chi}_{r}-\frac{\Delta \bar{s}}{2}\right)^{2}+\frac{(\Delta \bar{s})^{2}}{12}} \\
& \simeq\left|\mathcal{R}_{55}(s)\right| \sqrt{\left(1+\frac{\Delta \bar{s}}{2 \hat{\chi}(s)}\right)^{2}+\frac{(\Delta \bar{s})^{2}}{12 \chi^{2}(s)}}
\end{aligned}
$$

\section{APPENDIX E: INTEGRATION OVER THE $Z_{r 0}$ SPACE}

As discussed in Appendices C 2 and C 3, the retardation solutions $z_{r 0}^{( \pm)}$exist only for a finite range of $Z_{r 0}=$ $\left(x_{r 0}, x_{r 0}^{\prime}, \delta_{H r 0}\right)$ in the source particle's initial phase space, which is manifested in Eq. (C27) for $\chi_{r}^{2}<\beta_{0}^{2}$. To apply this condition to the evaluation of $\tilde{F}_{H}$ in Eq. (107) for a bunch with initial Gaussian intrinsic phase-space distribution, we first change the variables $\left(x_{r 0}, x_{r 0}^{\prime}, \delta_{H r 0}\right)$ to $\left(q_{1}, q_{2}, q_{3}\right)$, with $q_{3}$ linear to $\eta$ in Eq. (C8), and integrate $q_{1}$ and $q_{2}$ analytically. This procedure reduces the integral in Eq. (107) to a 2-dimensional integral over $s_{r}$ and $q_{3}$, which can be carried out numerically for arbitrary bunch parameters.

\section{Change of variables}

For the integration in Eq. (107), the limit in the space of $Z_{r 0}$, as given in Eq. (C27), can be made simpler by employing variable changes from $\left(x_{r 0}, x_{r 0}^{\prime}, \delta_{H r 0}\right)$ to $\left(q_{1}, q_{2}, q_{3}\right)$, with $q_{3}$ linearly related to $\eta$ in Eq. (C8). Here we choose

$$
q_{3}=\frac{\chi_{r} x_{r 1}-z_{r 1}}{\beta_{x 0}}
$$

which is included in the following transformation:

$$
\begin{aligned}
\left(\begin{array}{l}
q_{1} \\
q_{2} \\
q_{3}
\end{array}\right) & =J\left(s_{r}\right)\left(\begin{array}{c}
x_{r 0} / \beta_{x 0} \\
x_{r 0}^{\prime} \\
\delta_{H r 0}
\end{array}\right) \\
\text { for } J\left(s_{r}\right) & =\left(\begin{array}{lll}
J_{11} & J_{12} & J_{13} \\
J_{21} & J_{22} & J_{23} \\
J_{31} & J_{32} & J_{33}
\end{array}\right),
\end{aligned}
$$

where

$$
\begin{aligned}
& \mathbf{n}=\left(\begin{array}{c}
J_{31} \\
J_{32} \\
J_{33}
\end{array}\right)=\left(\begin{array}{c}
\chi_{r} \mathcal{R}_{11}\left(s_{r}\right)-\mathcal{R}_{51}\left(s_{r}\right) \\
\left(\chi_{r} \mathcal{R}_{12}\left(s_{r}\right)-\mathcal{R}_{52}\left(s_{r}\right)\right) / \beta_{x 0} \\
1 /\left(u \beta_{x 0}\right)
\end{array}\right), \\
& \mathbf{t}_{2}=\left(\begin{array}{c}
J_{21} \\
J_{22} \\
J_{23}
\end{array}\right)=\left(\begin{array}{c}
\mathcal{R}_{12}\left(s_{r}\right) / \beta_{x 0} \\
-\mathcal{R}_{11}\left(s_{r}\right) \\
-u \mathcal{R}_{16}\left(s_{r}\right)
\end{array}\right),
\end{aligned}
$$

with $\mathbf{t}_{2} \cdot \mathbf{n}=0$, and

$$
\mathbf{t}_{1}=\mathbf{t}_{2} \times \mathbf{n}=\left(\begin{array}{l}
J_{11} \\
J_{12} \\
J_{13}
\end{array}\right)=\left(\begin{array}{l}
J_{22} J_{33}-J_{23} J_{32} \\
J_{23} J_{31}-J_{21} J_{33} \\
J_{21} J_{32}-J_{22} J_{31}
\end{array}\right) .
$$

The idea here is that $\mathbf{n}$ is the normal vector of planes in the $Z_{r 0}$ space represented by $q_{3}=\mathbf{n} \cdot\left(x_{r 0}, x_{r 0}^{\prime}, \delta_{H r 0}\right)=q_{c}$, with $q_{c}$ real constants, and the vectors $\mathbf{t}_{1}$ and $\mathbf{t}_{2}$ are two orthogonal vectors parallel to the $q_{3}=q_{c}$ planes. Hence, Eq. (C27) requires the integral over $Z_{r 0}$ to be carried out in all the planes with $q_{3}=q_{c} \leq \eta_{\min }\left(x, z, s, s_{r}\right)\left|R_{0}\right| / \beta_{x 0}$.

Note that the vectors $\left(\mathbf{t}_{1}, \mathbf{t}_{2}, \mathbf{n}\right)$ are not unit vectors. The volume spanned by these vectors is

$$
\begin{aligned}
\Delta_{J}\left(s_{r}\right)= & \operatorname{det}\left[J\left(s_{r}\right)\right] \\
= & \frac{\mathcal{U}\left(s_{r}\right)}{\beta_{x 0}}\left[\frac{\mathcal{U}\left(s_{r}\right)}{u^{2} \beta_{x}\left(s_{r}\right)}+\left[\chi_{r} \sqrt{\beta_{x}\left(s_{r}\right)}+S\left(s_{r}\right) \sin \psi\left(s_{r}\right)\right.\right. \\
& \left.\left.+C\left(s_{r}\right) \cos \psi\left(s_{r}\right)\right]^{2}\right]
\end{aligned}
$$

with

$$
\begin{gathered}
\psi(s)=\int_{0}^{s} \frac{d s^{\prime}}{\beta\left(s^{\prime}\right)}, \quad S(s)=\int_{0}^{s} \frac{d s^{\prime}}{R\left(s^{\prime}\right)} \sqrt{\beta\left(s^{\prime}\right)} \sin \psi\left(s^{\prime}\right), \\
C(s)=\int_{0}^{s} \frac{d s^{\prime}}{R\left(s^{\prime}\right)} \sqrt{\beta\left(s^{\prime}\right)} \cos \psi\left(s^{\prime}\right)
\end{gathered}
$$

and

$$
\mathcal{U}\left(s_{r}\right)=\frac{\beta_{x}\left(s_{r}\right)}{\beta_{x 0}}+\left[u R_{16}\left(s_{r}\right)\right]^{2} .
$$

As a result, we have

$$
d Z_{r 0}=d x_{r 0} d x_{r 0}^{\prime} d \delta_{H r 0}=\frac{\beta_{x 0}}{\Delta_{J}\left(s_{r}\right)} d q_{1} d q_{2} d q_{3} .
$$

The inverse transformation of Eq. (E2) is 


$$
\left(\begin{array}{c}
x_{r 0} / \beta_{x 0} \\
x_{r 0}^{\prime} \\
\delta_{H r 0}
\end{array}\right)=J^{-1}\left(s_{r}\right)\left(\begin{array}{l}
q_{1} \\
q_{2} \\
q_{3}
\end{array}\right) .
$$

\section{Reduction of integration for the evaluation of $\tilde{F}_{H}(X, s)$}

Here by changing variables as discussed above, we will show that $\tilde{F}_{H}(X, s)$ in Eq. (107) can be reduced to a twodimensional integral, which allows further numerical integration.

With $\eta_{\min }$ and $\eta_{\max }$ given by Eq. (C14), let us define

$$
\begin{aligned}
& q_{\min }=q_{\min }\left(x, z, s, s_{r}\right)=\frac{\left|R_{0}\right|}{\beta_{x 0}} \eta_{\min }, \\
& q_{\max }=q_{\max }\left(x, z, s, s_{r}\right)=\frac{\left|R_{0}\right|}{\beta_{x 0}} \eta_{\max }
\end{aligned}
$$

and

$$
p\left(s_{r}, q_{3}\right)=p\left(x, z, s, s_{r}, q_{3}\right)=\sqrt{\left(q_{\min }-q_{3}\right)\left(q_{\max }-q_{3}\right)} .
$$

Then, for $W_{H}$ in Eq. (104), Eq. (107) can be rewritten as

$\tilde{F}_{H}(X, s) \simeq \frac{\beta_{0}^{2} N_{e} r_{e}\left|R_{0}\right|}{\gamma_{0} \beta_{x 0}} \int_{-\infty}^{\infty} d \Delta s \frac{-(\Delta s)^{2} / 2}{\left|u R_{16}\left(s_{r}\right)\right|}\left(I^{(+)}+I^{(-)}\right)$,

where for $q_{3}$ and $\Omega^{( \pm)}$respectively given by Eqs. (E1) and (76) we have

$$
I^{( \pm)}=\int_{\Omega^{( \pm)}} \frac{d Z_{r 0}}{p\left(s_{r}, q_{3}\right)} K^{( \pm)}
$$

and

$$
K^{( \pm)}=\left[\rho_{\mathrm{un}}\left(Z_{r 0}\right) \frac{\partial \lambda_{0}\left(z_{r 0}^{( \pm)}\right)}{\partial z_{r 0}}-u \lambda_{0}\left(z_{r 0}^{( \pm)}\right) \frac{\partial \rho_{\mathrm{un}}\left(Z_{r 0}\right)}{\partial\left(\delta_{H}\right)_{r 0}}\right] .
$$

For an initial Gaussian distribution given in Eqs. (109) and (110), one can perform Fourier transform for $\rho_{\text {un }}\left(Z_{0}\right)$ from $Z_{r 0}^{\prime}=\left(x_{r 0} / \beta_{x 0}, x_{r 0}^{\prime}, \delta_{H r 0}\right)$ to $k=\left(k_{1}, k_{2}, k_{3}\right)$ space, and perform similar transform for $\lambda\left(z_{r 0}\right)$ from $z_{r 0}$ to $k_{z}$. This gives

$$
\begin{aligned}
K^{( \pm)}= & \frac{1}{\beta_{x 0}(2 \pi)^{4} \sigma_{z 0}^{2}} \int_{-\infty}^{\infty} d^{3} k \exp \left(-\frac{1}{2} k \cdot D \cdot k+i k \cdot Z_{r 0}^{\prime}\right) \\
& \times \int_{-\infty}^{\infty} d \tilde{k}_{z}\left(i \tilde{k}_{z}-i \tilde{u} k_{3}\right) \exp \left(-\frac{\tilde{k}_{z}^{2}}{2}+i \tilde{k}_{z} \tilde{z}_{r 0}^{( \pm)}\right),
\end{aligned}
$$

with

$$
D=\left(\begin{array}{ccc}
\sigma_{x^{\prime} 0}^{2} & 0 & 0 \\
0 & \sigma_{x^{\prime} 0}^{2} & 0 \\
0 & 0 & \sigma_{H 0}^{2}
\end{array}\right), \quad \tilde{k}_{z}=k_{z} \sigma_{z 0}
$$

$$
\tilde{u}=u \sigma_{z 0} \text {, }
$$

and

$$
\tilde{z}_{r 0}^{( \pm)}=\frac{\left|R_{0}\right|}{\sigma_{z 0}} \bar{z}_{r 0}^{( \pm)}\left(x, z, s, s_{r}, Z_{r 0}\right)
$$

for $\bar{z}_{r 0}^{( \pm)}$given in Eq. (69).

Next, we apply the change of variables discussed in Appendix E 1 to the integrals in Eqs. (E13) and (E14), in the manner of Eq. (E9) and

$$
\left(\begin{array}{l}
k_{1} \\
k_{2} \\
k_{3}
\end{array}\right)=\left[J\left(s_{r}\right)\right]^{T}\left(\begin{array}{l}
k_{1}^{\prime} \\
k_{2}^{\prime} \\
k_{3}^{\prime}
\end{array}\right) .
$$

With the new variables, $\tilde{z}_{r 0}^{( \pm)}$in Eq. (E17) becomes

$$
\tilde{z}_{r 0}^{( \pm)}=\mu^{( \pm)}\left(x, s, z, s_{r}, q_{3}\right)+\nu \cdot q,
$$

where we define

$$
\begin{aligned}
\nu_{c}\left(s_{r}\right)= & \frac{1}{\sigma_{z 0}\left(1-\chi_{r}^{2}\right) u R_{16}\left(s_{r}\right)}, \\
\nu_{0}\left(x, z, s, s_{r}\right)= & \nu_{c}\left(s_{r}\right)\left[\Delta s\left(\chi_{r}-\frac{\Delta s}{2 R_{0}}\right)+\left(x-\chi_{r} z\right)\right], \\
\nu_{i}\left(s_{r}\right)= & \nu_{c}\left(s_{r}\right)\left\{\beta_{x 0}\left[\chi_{r} \mathcal{R}_{51}\left(s_{r}\right)-\mathcal{R}_{11}\left(s_{r}\right)\right]\left[J^{-1}\left(s_{r}\right)\right]_{1 i}+\left[\chi_{r} \mathcal{R}_{52}\left(s_{r}\right)-\mathcal{R}_{12}\left(s_{r}\right)\right]\left[J^{-1}\left(s_{r}\right)\right]_{2 i}\right. \\
& \left.+\left[\chi_{r} \mathcal{R}_{56}\left(s_{r}\right)-\mathcal{R}_{16}\left(s_{r}\right)\right]\left[J^{-1}\left(s_{r}\right)\right]_{3 i}\right\}, \\
\nu_{p}\left(s_{r}\right)= & \nu_{c}\left(s_{r}\right) \beta_{0} \beta_{x 0},
\end{aligned}
$$

with $i=1,2,3$,

$$
\begin{aligned}
\mu^{( \pm)}\left(x, z, s, s_{r}, q_{3}\right)= & \nu_{0}\left(x, z, s, s_{r}\right)+\nu_{3}\left(s_{r}\right) q_{3} \\
& \pm \nu_{p}\left(s_{r}\right) p\left(s_{r}, q_{3}\right),
\end{aligned}
$$

and

$$
\nu\left(s_{r}\right)=\left(\nu_{1}, \nu_{2}\right), \quad q=\left(q_{1}, q_{2}\right) .
$$

As a result, Eq. (E13) becomes 


$$
\begin{aligned}
I^{( \pm)}= & \frac{1}{(2 \pi)^{4} \sigma_{z 0}^{2}} \int_{-\infty}^{q_{\min }} \frac{d q_{3}}{p\left(s_{r}, q_{3}\right)} \int_{-\infty}^{\infty} d q_{1} \int_{-\infty}^{\infty} d q_{2} \\
& \times \int_{-\infty}^{\infty} d^{3} k^{\prime} \exp \left(-\frac{k^{\prime} \cdot S \cdot k^{\prime}}{2}+i k^{\prime} \cdot q\right) \\
& \times \int_{-\infty}^{\infty} d \tilde{k}_{z} i\left(\tilde{k}_{z}-\tilde{u}\left\{\left[J\left(s_{r}\right)\right]^{T} k^{\prime}\right\}_{3}\right) \\
& \times \exp \left[-\frac{\tilde{k}_{z}^{2}}{2}+i \tilde{k}_{z}\left(\mu^{( \pm)}+\nu \cdot q\right)\right]
\end{aligned}
$$

for

$$
S\left(s_{r}\right)=J\left(s_{r}\right) D\left[J\left(s_{r}\right)\right]^{T} .
$$

Note that, compared to the retardation constraint $\Omega^{( \pm)}$in Eq. (76) in the $Z_{r 0}$ space, the variable transformation now provides us in Eq. (E23) an explicit limit in the $q_{3}$ integral. Also notice that, due to Eqs. (C16) and (C17), the dependence of $q_{\min }$ and $q_{\max }$ on $(x, z, s)$ and $s_{r}$ for $\Delta s>0$ is different from that for $\Delta s<0$.

With the definition of

$$
\begin{gathered}
\Lambda\left(s_{r}\right)=\left(\begin{array}{cc}
S_{11} & S_{12} \\
S_{21} & S_{22}
\end{array}\right), \quad \theta_{s}\left(s_{r}\right)=\left(S_{13}, S_{23}\right), \\
j\left(s_{r}\right)=\left(J_{13}, J_{23}\right), \\
Y\left(s_{r}\right)=\left(\begin{array}{cc}
1+\nu \cdot \Lambda \cdot \nu & -\theta_{s} \cdot \nu \\
-\theta_{s} \cdot \nu & S_{33}
\end{array}\right),
\end{gathered}
$$

and

$$
\begin{aligned}
& \zeta\left(s_{r}\right)=\left[1+\tilde{u}(j \cdot \nu),-\tilde{u} J_{33}\right], \\
& \psi^{( \pm)}\left(x, z, s, s_{r}, q_{3}\right)=\left(\mu^{( \pm)}, q_{3}\right),
\end{aligned}
$$

further integration of Eq. (E23) yields

$$
I^{( \pm)}=\frac{-1}{\left(2 \pi \sigma_{z 0}\right)^{2}} \frac{1}{\sqrt{\operatorname{det} \Upsilon\left(s_{r}\right)}} \int_{-\infty}^{q_{\min }} \frac{d q_{3}}{p\left(s_{r}, q_{3}\right)} I_{0}^{( \pm)}
$$

for

$$
\begin{aligned}
I_{0}^{( \pm)}\left(x, z, s, s_{r}, q_{3}\right)= & \zeta \cdot \Upsilon^{-1} \cdot \psi^{( \pm)} \\
& \times \exp \left(-\frac{\psi^{( \pm)} \cdot \Upsilon^{-1} \cdot \psi^{( \pm)}}{2}\right) .
\end{aligned}
$$

Substituting Eq. (E28) into Eq. (E12), one gets the final results of $\tilde{F}_{H}$ :

$$
\begin{aligned}
\tilde{F}_{H}(x, z, s)= & C_{H} \int_{-\infty}^{\infty} d \Delta \bar{s} \frac{\Delta \bar{s}^{2}}{\left|u R_{16}\left(s_{r}\right)\right| \sqrt{\operatorname{det} Y\left(s_{r}\right)}} \\
& \times \int_{-\infty}^{q_{\min }} \frac{d q_{3}}{p\left(s_{r}, q_{3}\right)}\left[I_{0}^{(+)}+I_{0}^{(-)}\right]
\end{aligned}
$$

with

$$
C_{H}=\frac{\beta_{0}^{2} N r_{e}}{\gamma_{0} 4 \pi \sigma_{z 0}^{2}} \frac{\left|R_{0}\right|}{\beta_{x 0}} .
$$

It turns out that the behavior of the effective CSR force is largely impacted by how the cutoff value for $q_{3}$ in Eq. (E30), $q_{\min }\left(x, z, s, s_{r}\right)$, is compared to the rms value $\sigma_{q}\left(s_{r}\right)$ of $q_{3}$. Here $\sigma_{q}\left(s_{r}\right)=\left|R_{0}\right| \sigma_{\eta} / \beta_{x 0}$ for $\sigma_{\eta}$ in Eq. (88), or, from Eq. (E1),

$$
\sigma_{q}\left(s_{r}\right)=\sqrt{\left[J_{31}^{2}\left(s_{r}\right)+J_{32}^{2}\left(s_{r}\right)\right] \sigma_{x^{\prime} 0}^{2}+J_{33}^{2}\left(s_{r}\right) \sigma_{H 0}^{2}} .
$$

To make this comparison more explicit, we define

$$
\left(\tilde{q}_{3}, \tilde{q}_{\min }, \tilde{q}_{\max }, \tilde{p}\right)=\frac{1}{\sigma_{q}\left(s_{r}\right)}\left(q_{3}, q_{\min }, q_{\max }, p\right) .
$$

Furthermore, we change the variable from $\tilde{q}_{3}$ to $\tilde{p}$ by

$$
\tilde{q}_{3}(\tilde{p})=\tilde{q}_{\mathrm{ave}}-\sqrt{\left(\frac{\Delta \tilde{q}}{2}\right)^{2}+\tilde{p}^{2}},
$$

as obtained from Eq. (E11) for

$$
\tilde{q}_{\mathrm{ave}}=\frac{\tilde{q}_{\min }+\tilde{q}_{\max }}{2} \text { and } \Delta \tilde{q}=\tilde{q}_{\max }-\tilde{q}_{\min }
$$

This yields

$$
\begin{aligned}
\tilde{F}_{H}(x, z, s)= & C_{H}(s) \int_{-\infty}^{\infty} d \Delta \bar{s} \frac{\Delta \bar{s}^{2}}{\left|u R_{16}\left(s_{r}\right)\right| \sqrt{\operatorname{det} \Upsilon\left(s_{r}\right)}} \\
& \times \int_{0}^{\infty} \frac{d \tilde{p}}{\sqrt{(\Delta \tilde{q} / 2)^{2}+\tilde{p}^{2}}}\left(I_{0}^{(+)}+I_{0}^{(-)}\right)
\end{aligned}
$$

with $I_{0}^{( \pm)}$in Eq. (E29) replaced by $I_{0}^{( \pm)}\left[x, z, s, s_{r}\right.$, $\left.\sigma_{q}\left(s_{r}\right) \tilde{q}_{3}(\tilde{p})\right]$. One can show that $S_{33}\left(s_{r}\right)=\left[\sigma_{q}\left(s_{r}\right)\right]^{2}$ and thus in Eq. (E36) $\sqrt{\operatorname{det} \Upsilon\left(s_{r}\right)} \simeq \sigma_{q}\left(s_{r}\right)$.

[1] E. L. Saldin, E. A. Schneidmiller, and M. V. Yurkov, Nucl. Instrum. Methods Phys. Res., Sect. A 483, 516 (2002).

[2] S. Heifets, S. Krinsky, and G. Stupakov, Phys. Rev. ST Accel. Beams 5, 064401 (2002).

[3] Z. Huang and K. Kim, Phys. Rev. ST Accel. Beams 5, 074401 (2002).

[4] M. Borland et al., Nucl. Instrum. Methods Phys. Res., Sect. A 483, 268 (2002).

[5] Y.S. Derbenev, R. Rossbach, E. L. Saldin, and V.D. Shiltsev, DESY-TESLA-FEL-95-05, 1995.

[6] J. B. Murphy, S. Krinsky, and R. L. Gluckstern, Part. Accel. 57, 9 (1997).

[7] E. L. Saldin, E. A. Schneidmiller, and M. V. Yurkov, Nucl. Instrum. Methods Phys. Res., Sect. A 398, 373 (1997).

[8] Ya.S. Derbenev and V.D. Shiltsev, SLAC-Pub-7181, 1996. 
[9] M. Dohlus, "Field Calculation for Bunch Compressors," http://www.desy.de/csr, the CSR Workshop in DESYZeuthen, Berlin, 2002.

[10] R. Talman, Phys. Rev. Lett. 56, 1429 (1986).

[11] B. E. Carlsten, Phys. Rev. E 54, 838 (1996).

[12] R. Li and Ya. Derbenev, JLAB-TN-02-054, 2002.

[13] J.D. Jackson, Classical Electrodynamics (Wiley, New York, 1975), 2nd ed.

[14] C. Wang and A. Chao, SLAC/AP-100, 1995.

[15] R. Ruth, AIP Conf. Proc. No. 153 (AIP, New York, 1987), Vol. I.

[16] G. Geloni, E. Saldin, E. Schneidmiller, and M. Yurkov, http://arxiv.org/abs/physics/0310133, 2003.

[17] R. Li and Ya. S. Derbenev, in Proceedings of 2005 Particle Accelerator Conference, Knoxville, 2005.

[18] This terminology is adopted from Ref. [23].

[19] The definitions of the "parasitic compression" and the "critical compression" were from D. Douglas.
[20] T. Shintake, Proceedings of 2002 Linac Conference, Gyeongju, p. 663.

[21] G. Stupakov, Proceedings of 1997 PAC, p. 1688.

[22] R. Li, Nucl. Instrum. Methods Phys. Res., Sect. A 429, 310 (1999).

[23] R. Warnock, G. Bassi, and J. A. Ellison, Nucl. Instrum. Methods Phys. Res., Sect. A 558, 85 (2006).

[24] G. Bassi, J.A. Ellison, and K. Heinemann, Proceedings EPAC 2006, Edinburgh.

[25] G. Bassi, J. A. Ellison, K. Heinemann, and R. Warnock, Proceedings of PAC 2007 Albuquerque.

[26] Z. Huang and R. D. Ruth, Phys. Rev. Lett. 80, 2318 (1998).

[27] E. L. Saldin, E. A. Schneidmiller, and M. V. Yurkov, Nucl. Instrum. Methods Phys. Res., Sect. A 528, 355 (2004).

[28] T. Shaftan and Z. Huang, Phys. Rev. ST Accel. Beams 7, 080702 (2004). 